\title{
Scaling and diffusion of Dirac composite fermions
}

\author{
Chao-Jung Lee $\odot$ \\ Department of Physics, California Institute of Technology, Pasadena, California 91125, USA \\ Michael Mulligan \\ Department of Physics and Astronomy, University of California, Riverside, California 92511, USA
}

(Received 29 January 2020; accepted 25 March 2020; published 8 June 2020)

\begin{abstract}
We study the effects of quenched disorder and a dissipative Coulomb interaction on an anyon gas in a periodic potential undergoing a quantum phase transition. We use a $(2+1)$-dimensional low-energy effective description that involves $N_{f}=1$ Dirac fermion coupled to a $U(1)$ Chern-Simons gauge field at level $(\theta-1 / 2)$. When $\theta=1 / 2$ the anyons are free Dirac fermions that exhibit an integer quantum Hall transition; when $\theta=1$ the anyons are bosons undergoing a superconductor-insulator transition in the universality class of the three-dimensional XY model. Using the large $N_{f}$ approximation we perform a renormalization-group analysis. We find the Coulomb interaction to be an irrelevant perturbation of the clean fixed point for any $\theta$. The dissipative Coulomb interaction allows for two classes of IR stable fixed points in the presence of disorder: those with a finite nonzero Coulomb coupling and dynamical critical exponent $z=1$ and those with an effectively infinite Coulomb coupling and $1<z<2$. At $\theta=1 / 2$ the clean fixed point is stable to charge-conjugation preserving (random mass) disorder, while a line of diffusive fixed points is obtained when the product of charge-conjugation and time-reversal symmetries is preserved. At $\theta=1$ we find a finite disorder fixed point with unbroken charge-conjugation symmetry whether or not the Coulomb interaction is present. Other cases result in runaway flows. We comment on the relation of our results to other theoretical studies and the relevancy to experiment.
\end{abstract}

DOI: 10.1103/PhysRevResearch.2.023303

\section{INTRODUCTION}

Delocalization transitions determine the phase diagrams of various electronic systems [1-3]. In three spatial dimensions, such transitions can occur between a diffusive metal and a localized insulator. In two dimensions (and fewer), localization generally relegates $T=0$ metallic states to isolated critical points. The integer quantum Hall transition (IQHT) and the superconductor-insulator transition (SIT) are prototypical examples of such two-dimensional diffusive quantum critical points, having been well characterized by extensive experimental and numerical work over the past 30 years (see [4-7] and references therein). Nevertheless, our understanding of these quantum states remains incomplete.

Theories of noninteracting electrons have provided valuable insight to the IQHT [8]. As the critical point is approached by tuning the external magnetic field or electron density to criticality $\delta \rightarrow 0$, the localization length is found to diverge as $|\delta|^{-v}$ with $v=2.593(5)$ [9], while $v_{\text {expt }} \approx$ 2.38 experimentally [5,6]. A diverging timescale $\xi_{t} \sim \xi^{-v z}$ is also expected near the quantum critical point. Theories of noninteracting electrons yield a dynamical critical exponent

Published by the American Physical Society under the terms of the Creative Commons Attribution 4.0 International license. Further distribution of this work must maintain attribution to the author(s) and the published article's title, journal citation, and DOI. $z=2[8,10,11] ; z_{\text {expt }} \approx 1[4,6]$ (although see [12]). (For the magnetic field-tuned SIT, $v_{\text {expt }} \approx 4 / 3$ or $\approx 7 / 3$ and $z_{\text {expt }} \approx 1$ experimentally [13].) The challenge is to develop a framework that combines the effects of electron interactions with those of disorder [14].

Duality is a powerful tool for understanding the behavior of strongly interacting systems. Recent work has uncovered a duality web that relates various $(2+1)$ D relativistic quantum field theories (see [15] and references therein). Included in this set are simple, toy models for integer quantum Hall and superconductor-insulator transitions. In this paper, we study the effects of quenched disorder and a dissipative Coulomb interaction on the critical properties of two such models. The hope is to abstract lessons that may be valid more generally. As we discuss, these theories have a rich set of random critical behaviors.

For the first member of the duality web, consider a system of spinless electrons hopping on a square lattice with a half unit of magnetic flux penetrating each plaquette [16] ([17] may alternatively be considered). An IQHT is obtained as the ratio of the (staggered) chemical potential to next-neighbor hopping is varied. The critical properties of the transition are controlled by a free Dirac fermion $\Psi$ with the Lagrangian:

$$
\mathcal{L}_{\text {Dirac }}=\bar{\Psi} i \not D_{A} \Psi-M \bar{\Psi} \Psi+\frac{1}{2} \frac{1}{4 \pi} A d A,
$$

where $A_{\mu}$ is a nondynamical (external) $U(1)$ gauge field and the Chern-Simons term $A d A=\epsilon^{\mu \nu \rho} A_{\mu} \partial_{\nu} A_{\rho}$. (Additional details for the Lagrangians appearing in this section are given 
in Sec. II.) The mass $M$ vanishes at criticality. In the presence of an external magnetic field, (1.1) describes the particle-hole (PH) symmetric limit of the half-filled zeroth/lowest Landau level of Dirac/nonrelativistic electrons [18]. In this paper, we consider vanishing magnetic field. A dual effective theory to (1.1) consists of a Dirac fermion $\psi$ coupled to a dynamical (emergent) $U$ (1) gauge field $a_{\mu}$ :

$$
\mathcal{L}_{\mathrm{F}}=\bar{\psi} i \not D_{a} \psi-m \bar{\psi} \psi-\frac{1}{2} \frac{1}{2 \pi} a d A+\frac{1}{2} \frac{1}{4 \pi} A d A-\frac{1}{4} f_{\mu \nu}^{2},
$$

where the mass $m \propto M$ and the field strength $f_{\mu \nu}=\partial_{\mu} a_{\nu}-$ $\partial_{\nu} a_{\mu}$. (We use "condensed matter" notation when writing these Lagrangians; see [19] for a precise explanation of the meaning of, e.g., Chern-Simons terms with half-integer levels.) Equation (1.2) was first introduced as a dual description of the half-filled Landau level [18] or the gapless surface state of a time-reversal invariant topological insulator [20,21] (when the $A d A$ term is absent) with $\psi$ being the Dirac composite fermion; its inclusion in the duality web was explained in $[19,22,23]$. When the external magnetic field is zero, the Dirac composite fermion chemical potential sits at the Dirac point.

For the second member of the duality web, consider a collection of repulsive bosons in a periodic potential [24]. For commensurate filling, the system exhibits a superfluid to Mott insulator transition with a charge-conjugation symmetry as the ratio of the boson hopping strength to repulsion is tuned. The long-wavelength critical properties are described by the $3 \mathrm{D}$ XY model:

$$
\mathcal{L}_{\mathrm{XY}}=\left|D_{A} \Phi\right|^{2}-M|\Phi|^{2}-|\Phi|^{4} .
$$

(Broken charge-conjugation symmetry generally results in a term proportional to $\Phi^{*} i \partial_{t} \Phi$.) In mean-field theory, the $M<0$ region is a superfluid, while the $M>0$ region is an insulator; we will view (1.3) as describing a SIT. A dual effective theory [25-27] to (1.3) is

$$
\begin{aligned}
\mathcal{L}_{\mathrm{B}}= & \bar{\psi} i \not D_{a} \psi-m \bar{\psi} \psi+\frac{1}{2} \frac{1}{4 \pi} a d a-\frac{1}{2 \pi} a d A \\
& +\frac{1}{4 \pi} A d A-\frac{1}{4} f_{\mu \nu}^{2} .
\end{aligned}
$$

The statistics of the particles that (1.2) and (1.4) describe is controlled by the coefficient of the ada term.

Quenched disorder can have a profound effect on the nature of the above critical points and lead to new universality classes. Reference [16] considered the effects of quenched randomness on the free Dirac fermion fixed point in (1.1). While for generic disorder the theory flows to strong coupling, if only a random vector potential $\mathbf{A}(\mathbf{x})$ is present the theory features a line of diffusive fixed points characterized by a continuously variable dynamical exponent $z$; the clean fixed point is stable to random mass disorder $M(\mathbf{x})$. Ye and Sachdev [28] and Ye [29] generalized this study to fractional quantum Hall transitions in the presence of an unscreened Coulomb interaction using a model closely related to (1.4). Recently, Goswami, Goldman, and Raghu [30] and Thomson and Sachdev [31] considered the effects of randomness on (1.2) with $2 N_{f}$ fermion flavors. Using the large $N_{f}$ expan- sion, we reexamine these works and extend them to include the effects of a dissipative Coulomb interaction (Sec. II C) and "topological disorder" (Sec. II D), generally confirming prior results that found interacting, diffusive fixed points for certain types of disorder. Related works studying the effects of quenched randomness on theories of Dirac fermions coupled to a fluctuating boson include $[32,33]$.

In contrast to the fermion models, only random mass disorder $M(\mathbf{x})$ has resulted in accessible diffusive fixed points of the XY model. Early work [34-37] studying the $O\left(2 N_{f}\right)$ generalization of (1.3) used a double- $\epsilon$ expansion to find an interacting, finite disorder fixed point. However, the nature of the renormalization-group flow in the vicinity of the fixed point is peculiar, exhibiting an anomalously long "time" to achieve criticality. Recently, this problem was reexamined within a large $N_{f}$ expansion by Goldman, Thomson, $\mathrm{Nie}$, and $\mathrm{Bi}$ [38], where it was argued that the anomalous renormalization-group trajectories [34-37] are a relic of the double- $\epsilon$ expansion. Furthermore, [38] finds remarkable agreement with the critical exponents of the dirty XY model calculated by numerical simulation [39-42]. We consider this analysis from the perspective of the "fermionic dual" of the $\mathrm{XY}$ model in (1.4), providing qualitative confirmation of the renormalization-group flow found in [38]. To $\mathcal{O}\left(1 / N_{f}\right)$, we find a finite disorder fixed point with critical exponents:

$$
v^{-1}=1 \quad \text { and } \quad z=1+\frac{1.411}{N_{f}} ;
$$

$v=1$ and $z=1+0.54 / N_{f}$ is reported in [38]. We also consider other types of disorder that is sourced by the random gauge field $A_{\mu}(\mathbf{x})$.

The important influence of a Coulomb interaction on the critical properties of the above transitions was stressed long ago [43], where it was argued that an unscreened Coulomb interaction generically results in a dynamical critical exponent $z=1$. In addition, the observed IQHT and SIT appear to be sensitive to the precise nature of the Coulomb interaction $([44,45]$ and references therein). For example, a capacitively coupled screening plane has been found to affect the metallic behavior in thin films [46], lifting an anomalous lowtemperature metallic regime that intervenes a direct magnetic field-tuned SIT. To investigate such effects, we consider a Coulomb interaction that is screened by a diffusive twodimensional Fermi gas [47]. The dissipative Coulomb interaction that results allows for two types of fixed points: those with a finite nonzero Coulomb coupling and $z=1$ and those with an effectively infinite Coulomb interaction and $z \neq 1$ [48]. For the "fermionic dual" of the XY model with random mass disorder, we find critical exponents

$$
v^{-1}=1 \text { and } 1 \leqslant z<2
$$

with $z$ saturating the lower bound for the unscreened Coulomb interaction and varying continuously with an effective dissipation parameter for $z>1$. In our approach, we are unable to access the "infinite $z$ " fixed point of the dissipative XY model found in [49]. Our result differs from that of Vishwanath, Moore, and Senthil [47], who studied the effects of a dissipative Coulomb interaction on the dirty XY model using the double- $\epsilon$ expansion and found a line of fixed points with $z=1$ and continuously varying $v$. We also consider the effects 
of other types of disorder on the theories in (1.4) [and (1.2)] when a dissipative Coulomb interaction is present.

\section{MODEL OVERVIEW}

In this section, we introduce the effective model that realizes an IQHT/SIT and the critical properties of which we will analyze in Sec. III.

Consider the $(2+1) \mathrm{D}$ theory of $N_{f}$ Dirac fermions $\psi_{I}$ coupled to a $U(1)$ Chern-Simons gauge field $a_{\mu}$ at level $(\theta-1 / 2)$ :

$$
\begin{aligned}
\mathcal{L}^{(1)}= & \sum_{I=1}^{N_{f}} \bar{\psi}_{I}\left(i \not D_{a}-m\right) \psi_{I}-\frac{1}{2} \frac{1}{4 \pi} a d a \\
& +\frac{\theta}{4 \pi}(a-A) d(a-A)-\frac{1}{4} f_{\mu \nu}^{2} .
\end{aligned}
$$

The notation in (2.1) is as follows: $\bar{\psi}=\psi^{\dagger} \gamma^{0} ; \not D_{a}=\left(\partial_{\mu}-\right.$ $\left.i a_{\mu}\right) \gamma^{\mu}$ with $\mu \in\{0,1,2\}=\{t, x, y\}$, and a Chern-Simons term $A d A=\epsilon^{\mu v \rho} A_{\mu} \partial_{\nu} A_{\rho}$. For the purpose of discussing the symmetries of (2.1) later in this section, we choose Minkowski signature $\eta^{\mu \nu}=\operatorname{diag}(+1,-1,-1)$ and $\gamma$ matrices $\left(\gamma^{0}, \gamma^{1}, \gamma^{2}\right)=\left(\sigma^{3}, i \sigma^{1}, i \sigma^{2}\right)$ where $\sigma^{j}$ are the Pauli $\sigma$ matrices; in the renormalization-group analysis in Sec. III, we will work in Euclidean signature.

When $N_{f}=2 \theta=1$, we recover (1.2), the dual of a free Dirac fermion; when $N_{f}=\theta=1$, we find the dual (1.4) to the 3D XY model. Reminiscent of conventional flux attachment [50,51], $\theta^{-1}$ quantifies the number of attached flux quanta; for general $\theta, \mathcal{L}^{(1)}$ is the model for an anyon gas introduced by Chen, Fisher, and Wu [25]. We refer to $\psi_{I}$ as a Dirac composite fermion. $A_{\mu}$ is a nondynamical $U(1)$ gauge field that we identify with electromagnetism. In Sec. II C we give $A_{0}$ dynamics to discuss the Coulomb interaction. In Secs. II A and II B, where we discuss the phase diagram and symmetry of (2.1), we take $N_{f}=1$. Otherwise, $N_{f}$ is an arbitrary parameter that allows for analytic control of the interaction mediated by $a_{\mu}$ as $N_{f} \rightarrow \infty$.

\section{A. Mean-field phase diagram at $N_{f}=1$}

For a given $\theta$, the mean-field phase diagram of (2.1) at $N_{f}=1$ is parametrized by the Dirac composite fermion mass $m$. At energies less than $|m|$, we may integrate out the Dirac composite fermion to obtain the effective Lagrangian:

$$
\begin{aligned}
\mathcal{L}_{\text {eff }}= & \frac{\operatorname{sign}(m)-1+2 \theta}{2} \frac{1}{4 \pi} a d a-\frac{\theta}{2 \pi} a d A \\
& +\frac{\theta}{4 \pi} A d A-\frac{1}{4} f_{\mu \nu}^{2} .
\end{aligned}
$$

(By "integrate out," we refer to path-integral relations of the form $\int \mathcal{D} \phi e^{i \int\left(\frac{1}{2} \phi K \phi+\phi J\right)} \propto e^{i \int\left(-\frac{1}{2} J K^{-1} J\right)}$, where $\phi$ and $J$ are real fields and $K$ is some kernel, e.g., a kinetic term for $\phi$. Thus, we equate the Lagrangians $\frac{1}{2} \phi K \phi+\phi J=-\frac{1}{2} J K^{-1} J$ upon integrating out $\phi$. Such identities follow directly from the $\phi$ equation of motion when $\phi$ appears quadratically in the Lagrangian.) Higher-order terms in $a_{\mu}$ can be ignored as $|m| \rightarrow \infty$. The Maxwell term $f_{\mu \nu}^{2}$ can also be dropped in this long-wavelength analysis.

\section{1. $\theta=1 / 2$}

Setting $\theta=1 / 2$, there are two phases. For $m>0$ we find the effective Lagrangian for an insulator at zero temperature:

$$
\mathcal{L}_{\text {ins }}=\frac{1}{2}\left(\frac{1}{4 \pi} a d a-\frac{1}{2 \pi} a d A+\frac{1}{4 \pi} A d A\right)=0,
$$

where the second equality follows from integrating out $a_{\mu}$. For $m<0$ we find the long-wavelength Lagrangian for an integer Hall state:

$$
\begin{gathered}
\mathcal{L}_{\mathrm{IQH}}=\frac{1}{2}\left(-\frac{1}{4 \pi} a d a-\frac{1}{2 \pi} a d A+\frac{1}{4 \pi} A d A\right)=\frac{1}{4 \pi} A d A . \\
\text { 2. } \boldsymbol{\theta}=1
\end{gathered}
$$

Next set $\theta=1$. We again find the insulator when $m>0$ :

$$
\mathcal{L}_{\text {ins }}=\frac{1}{4 \pi} a d a-\frac{1}{2 \pi} a d A+\frac{1}{4 \pi} A d A=0 .
$$

To identify the $m<0$ phase, it is helpful to include the charge $e_{*}=q$ (measured in units of the electric charge $e$ ) carried by the boson $\Phi$ in (1.3) by substituting $A_{\mu} \rightarrow q A_{\mu}$ :

$$
\mathcal{L}_{\mathrm{SC}}=-\frac{q}{2 \pi} a d A+\frac{q^{2}}{4 \pi} A d A .
$$

Equation (2.6) describes a $\mathbb{Z} / q$ gauge theory, the longwavelength description of a superconductor with charge- $q$ condensate $[52,53]$.

\section{B. Discrete symmetry at $N_{f}=1$}

The types of randomness that can be added to (2.1) are characterized by charge-conjugation $\mathcal{C}$ and time-reversal $\mathcal{T}$ symmetries. (Parity, i.e., spatial reflection, is necessarily broken in the presence of quenched disorder.) These symmetries are defined with respect to the electron and boson Lagrangians in Eqs. (1.1) and (1.3). We discuss their implementation $[18,19,54-56]$ in the dual Lagrangian (2.1) at $N_{f}=1$ at criticality $m=0$.

$$
\text { 1. } \theta=1 / 2
$$

The free Dirac Lagrangian in (1.1) is invariant under charge conjugation $\mathcal{C}$ :

$$
\Psi \mapsto \sigma^{1} \Psi^{*}, \quad A_{\mu} \mapsto-A_{\mu} .
$$

The presence of the Chern-Simons term for $A_{\mu}$ reflects the violation of time reversal $\mathcal{T}$ :

$$
t \mapsto-t, \quad \Psi \mapsto-i \sigma^{2} \Psi, \quad\left(A_{0}, A_{i}\right) \mapsto\left(A_{0},-A_{i}\right),
$$

which is antiunitary $(i \mapsto-i)$. On the surface of a timereversal invariant topological insulator, this Chern-Simons term is absent and so $\mathcal{T}$ can be preserved.

The dual Lagrangian (2.1) at $\theta=1 / 2$ is also invariant under $\mathcal{C}$ :

$$
\psi \mapsto \sigma^{1} \psi, \quad a_{\mu} \mapsto-a_{\mu}, \quad A_{\mu} \mapsto-A_{\mu} .
$$


Identifying the electromagnetic currents across the duality between (1.1) and (2.1), $\frac{\delta \mathcal{L}_{\text {Dirac }}}{\delta A_{\mu}}=\frac{\delta \mathcal{L}^{(1)}}{\delta A_{\mu}}$, we equate

$$
\bar{\Psi} \gamma^{\mu} \Psi=\frac{1}{4 \pi} \epsilon^{\mu \nu \rho} \partial_{\nu} a_{\rho} .
$$

Similarly, the $a_{0}$ equation of motion relates

$$
\frac{1}{4 \pi} \epsilon^{\mu \nu \rho} \partial_{\nu} A_{\rho}=\bar{\psi} \gamma^{\mu} \psi
$$

Equations (2.10) and (2.11) imply that in (2.1), $\mathcal{C} T$,

$$
\begin{aligned}
& t \mapsto-t, \quad \psi \mapsto-i \sigma^{2} \psi^{*}, \quad\left(a_{0}, a_{i}\right) \mapsto\left(a_{0},-a_{i}\right), \\
& \left(A_{0}, A_{i}\right) \mapsto\left(-A_{0}, A_{i}\right) .
\end{aligned}
$$

Thus, $\mathcal{T}$ and $\mathcal{C} \mathcal{T}$ are exchanged across the duality: the $\mathcal{T}$ transformations on $\Psi$ and $A_{\mu}$ are identical to the $\mathcal{C} \mathcal{T}$ transformations on $\psi$ and $a_{\mu}$, and vice versa. In the absence of the Chern-Simons term for $A_{\mu},(2.1)$ is time-reversal invariant.

While the dual Lagrangians in (1.1) and (2.1) violate timereversal invariance as $(2+1) \mathrm{D}$ theories, they do preserve a "nonlocal" PH transformation. To define this, consider the following transformations of a general Lagrangian $L(A)$ which has a $U(1)$ symmetry current that is coupled to a nondynamical field $A_{\mu}$ [57]:

$$
\begin{aligned}
\mathbf{T}: L(A) & \mapsto L(A)+\frac{1}{4 \pi} A d A ; \\
\mathbf{S}: L(A) & \mapsto L(c)+\frac{1}{2 \pi} c d A .
\end{aligned}
$$

$\mathbf{T}$ shifts the Hall conductivity by a unit; $\mathbf{S}$ converts $A_{\mu}$ into a dynamical $U(1)$ gauge field $c_{\mu}$ and adds a BF term, which couples the field strength $d c$ to a new external field $A_{\mu}$. Equations (2.13) and (2.14) implement modular transformations on the conductivity tensor of the $U(1)$ symmetry current coupling to $A_{\mu}$. The PH transformation is defined as $\mathcal{T}$ followed by the modular $\mathbf{T}$ transformation (2.13). Notice that the Dirac masses $\bar{\Psi} \Psi$ and $\bar{\psi} \psi$ are odd under PH symmetry and even under $\mathcal{C}$.

$$
\text { 2. } \theta=1
$$

The XY model in (1.3) is invariant under charge conjugation $\mathcal{C}$

$$
\Phi \mapsto \Phi^{*}, \quad A_{\mu} \mapsto-A_{\mu},
$$

and time reversal $\mathcal{T}$ :

$$
t \mapsto-t, \quad \Phi \mapsto \Phi, \quad\left(A_{0}, A_{i}\right) \mapsto\left(A_{0},-A_{i}\right) .
$$

The dual Lagrangian in (2.1) at $\theta=1$ is only invariant under $\mathcal{C}$ defined in (2.9); it is not invariant under $\mathcal{T}$ :

$$
\begin{aligned}
& t \mapsto-t, \quad \psi \mapsto-\sigma^{3} \psi^{*}, \quad\left(a_{0}, a_{i}\right) \mapsto\left(-a_{0}, a_{i}\right), \\
& \left(A_{0}, A_{i}\right) \mapsto\left(A_{0},-A_{i}\right),
\end{aligned}
$$

with $i \mapsto-i$. Instead, time reversal is an emergent symmetry of the long-wavelength physics [19,54]. In addition, (2.1) is invariant under a "nonlocal" particle-vortex (PV) transformation:

$$
\begin{aligned}
& t \mapsto-t, \quad \psi \mapsto-i \sigma^{2} \psi, \quad\left(a_{0}, a_{i}\right) \mapsto\left(a_{0},-a_{i}\right), \\
& \left(A_{0}, A_{i}\right) \mapsto\left(-A_{0}, A_{i}\right),
\end{aligned}
$$

TABLE I. Charge-conjugation $\mathcal{C}$ and time-reversal $\mathcal{T}$ symmetry assignments of various operators.

\begin{tabular}{lcc}
\hline \hline & $\mathcal{C}$ & $\mathcal{T}$ \\
\hline $\bar{\psi} \psi$ & + & - \\
$\bar{\psi} \gamma_{0} \psi$ & - & - \\
$\bar{\psi} \gamma_{j} \psi$ & - & + \\
$a_{0}$ & - & - \\
$a_{j}$ & - & + \\
$b=\partial_{x} a_{y}-\partial_{y} a_{x}$ & - & + \\
$e_{j}=\partial_{0} a_{j}-\partial_{j} a_{0}$ & - & - \\
\hline \hline
\end{tabular}

followed by the modular $\mathbf{S}$ transformation (2.14). The PV transformation is analogous to the $\mathrm{PH}$ transformation of the previous section [58]; it maps the 3D XY model to its scalar quantum electrodynamics dual $[59,60]$, and vice versa.

Duality maps $|\Phi|^{2} \leftrightarrow \bar{\psi} \psi$. While it is clear that the Dirac mass is even under $\mathcal{C}$, it is less obvious that perturbation by $\bar{\psi} \psi$ is time-reversal invariant. This can be understood in the following sense: Perturbation of (2.1) by $\bar{\psi} \psi$ and of the time reversal of (2.1) [obtained using (2.17)] by $-\bar{\psi} \psi$ results in identical phases.

\section{Symmetry assignment summary}

Table I summarizes the transformations of the operators that appear in (2.1) under charge-conjugation $\mathcal{C}$ and timereversal $\mathcal{T}$ symmetries. We use these transformation assignments to characterize the types of randomness that may be added to $\mathcal{L}^{(1)}$ for general $N_{f}$.

\section{Dissipative Coulomb interaction}

\section{Dualizing the Coulomb interaction}

The Coulomb interaction between fermions/bosons carrying charge $e_{*}$ arises from the exchange of a dynamical $(3+1)$-dimensional electromagnetic scalar potential $A_{0}$. In Fourier space, we consider the action that couples a $(2+1) \mathrm{D}$ charge density $J_{0}\left(k_{0}, k\right)$ to the scalar potential $A_{0}\left(k_{0}, \vec{k}\right)$ :

$$
\begin{array}{r}
-\frac{1}{2 e_{*}^{2}} \int d^{4} k A_{0}\left(k_{0}, \vec{k}\right)\left(\vec{k}^{2}\right) A_{0}\left(-k_{0},-\vec{k}\right) \\
-\int d^{3} k J_{0}\left(k_{0}, k\right) \int d k_{3} A_{0}\left(-k_{0},-\vec{k}\right),
\end{array}
$$

where $k=\left(k_{1}, k_{2}\right)$ and $\vec{k}=\left(k_{1}, k_{2}, k_{3}\right) . J_{0}\left(k_{0}, k\right)$ is the Fourier transform of $\bar{\Psi} \gamma^{0} \Psi(x)$ for the free Dirac fermion (1.1) or $i \Phi^{*} \partial_{0} \Phi(x)-i\left(\partial_{0} \Phi^{*}\right) \Phi(x)$ for the XY model (1.3). The absence of an $A_{0} k_{0}^{2} A_{0}$ term means that $A_{0}$ mediates an instantaneous interaction, an approximation valid for particles moving at speeds much less than the photon velocity. Integrating out the $A_{0}$ field we find the unscreened Coulomb interaction,

$$
S_{\text {unscreened }}=-\frac{\pi}{2} \int d^{3} k J_{0}\left(k_{0}, k\right) \frac{e_{*}^{2}}{|k|} J_{0}\left(-k_{0},-k\right),
$$

between $(2+1) \mathrm{D}$ particles. It is convenient to interpret $S_{\text {unscreened }}$ as arising from the exchange of a purely $(2+1) \mathrm{D}$ 
gauge field $\tilde{A}_{0}$ with kinetic term and coupling to $J_{0}$ as

$$
\begin{aligned}
S_{\tilde{A}_{0}}= & -\int d^{3} k\left[\tilde{A}_{0}\left(k_{0}, k\right) \frac{|k|}{\pi e_{*}^{2}} \tilde{A}_{0}\left(-k_{0},-k\right)\right. \\
& \left.+J_{0}\left(k_{0}, k\right) \tilde{A}_{0}\left(-k_{0},-k\right)\right] .
\end{aligned}
$$

The electromagnetic charge density $J_{0}(x)$ dualizes in (2.1) according to

$$
J_{0}(x)=\frac{\delta \mathcal{L}^{(1)}}{\delta A_{0}}=-\frac{\theta}{2 \pi} \epsilon_{i j} \partial_{i} a_{j},
$$

for vanishing $A_{j}$. Decomposing the gauge field $a_{i}\left(k_{0}, k\right)=$ $i \frac{k_{i}}{|k|} a_{L}\left(k_{0}, k\right)-i \frac{k_{j}}{|k|} \epsilon_{j i} a_{T}\left(k_{0}, k\right)$ in terms of its longitudinal and transverse components, the (unscreened) Coulomb interaction becomes a kinetic term for $a_{T}$ [61],

$$
S_{\text {unscreened }}=-\frac{e_{*}^{2} \theta^{2}}{8 \pi} \int d^{3} k a_{T}\left(k_{0}, k\right)|k| a_{T}\left(-k_{0},-k\right),
$$

that dominates a possible Maxwell term for $a_{\mu}$ at long wavelengths. A similar transformation of the Coulomb interaction occurs in nonrelativistic composite fermion theories [62].

\section{Dissipation}

To model dissipation following [47], we consider an auxiliary system consisting of a parallel two-dimensional electron gas (2DEG) that is coupled to (2.1) through the Coulomb interaction, specifically, through $\tilde{A}_{0}$. The spatial separation between the system (2.1) and electron gas is ignored. The electron Green's function is assumed to take a diffusive form:

$$
G_{2 \mathrm{D}}^{-1}\left(i k_{0}, k\right)=i k_{0}-\left(\frac{k^{2}}{2 m}-\epsilon_{F}\right)+\frac{i}{2 \tau} \operatorname{sign}\left(k_{0}\right) .
$$

The dissipative effects arising from the coupling to the twodimensional electron gas are encoded in a correction to the $\tilde{A}_{0}$ kinetic term in $S_{\tilde{A}_{0}}[63,64]$ :

$$
\delta S_{\tilde{A}_{0}}=\int d^{3} k \tilde{A}_{0}\left(k_{0}, k\right) \frac{\sigma_{e} k^{2}}{\left|k_{0}\right|+D_{e} k^{2}} \tilde{A}_{0}\left(-k_{0},-k\right),
$$

where the Drude conductivity $\tilde{\sigma}_{e}=q_{e}^{2} N D_{e}$ with $N$ the density of states at Fermi energy $\epsilon_{F}$ of the two-dimensional electron gas and $D_{e}$ its diffusivity. Higher-order corrections due to the two-dimensional electron gas will be ignored. Including $\delta S_{\tilde{A}_{0}}$ we obtain the dissipation-corrected density-density (2.22) interaction upon integrating out $\tilde{A}_{0}$ :

$S^{(2)}=-\frac{e_{*}^{2} \theta^{2}}{8 \pi} \int d^{3} k a_{T}\left(k_{0}, k\right)\left(\frac{k^{2}}{|k|+f\left(k_{0}, k\right)}\right) a_{T}\left(-k_{0},-k\right)$,

where

$$
f\left(k_{0}, k\right)=\frac{\sigma_{e} k^{2}}{\left|k_{0}\right|+D_{e} k^{2}}, \quad \sigma_{e}=e_{*}^{2} \tilde{\sigma}_{e} .
$$

We recover the dual of an unscreened Coulomb interaction when $q_{e}=0$, as expected, or as $|k| /\left|k_{0}\right| \rightarrow 0$. The Coulomb interaction is short ranged as $D_{e} \rightarrow \infty$ at finite density of states $N$ or when $\left|k_{0}\right| /|k| \rightarrow 0$; in either of these limits, we find a Maxwell-like kinetic term for $a_{T}$ (albeit with inverted charge $\left.1 / e_{*}\right)$.

\section{Quenched randomness}

We consider the effects of quenched disorder that is induced by random $A_{\mu}(\mathbf{x})$ and $M(\mathbf{x})$. In this discussion, we assume the Coulomb interaction has been included via (2.26) and $A_{\mu}(\mathbf{x})$ is a nondynamical quenched random variable. Since $m(\mathbf{x}) \propto M(\mathbf{x})$, these perturbations readily map across the duality to

$$
\delta \mathcal{L}=-m(\mathbf{x}) \bar{\psi} \psi(x)-\frac{\theta}{2 \pi} A(\mathbf{x}) d a(x),
$$

where $\mathbf{x}=\left(x_{1}, x_{2}\right)$ and $x=\left(x_{0}, x_{1}, x_{2}\right)$. The second term in Eq. (2.28) is "topological disorder," i.e., a random source to the field strength or "topological" current $d a$. We have dropped a possible term proportional to $\epsilon_{i j} A_{0}(\mathbf{x}) \partial_{i} A_{j}(\mathbf{x})$ arising from the Chern-Simons term for $A_{\mu}$ in (2.1).

Interactions generate additional operators with random couplings, consistent with the symmetries of $A_{\mu}(\mathbf{x})$ and $m(\mathbf{x})$. The (quantum) Harris criterion [65] for Gaussian-correlated randomness implies the relevant terms at low energies correspond to operators with scaling dimensions $\Delta \leqslant z+1$. At large $N_{f}[25,66,67]$ the most generic random terms to include are [31]

$$
\begin{aligned}
\mathcal{L}_{\mathrm{dis}}= & m(\mathbf{x}) \bar{\psi} \psi(x)+i \tilde{a}_{0}(\mathbf{x}) \bar{\psi} \gamma^{0} \psi(x)+i \tilde{a}_{j}(\mathbf{x}) \bar{\psi} \gamma^{j} \psi(x) \\
& -A_{0}(\mathbf{x}) b(x)+\epsilon_{j k} A_{j}(\mathbf{x}) e_{k}(x),
\end{aligned}
$$

where $b=\epsilon_{i j} \partial_{i} a_{j}$ and $e_{k}=\partial_{0} a_{k}-\partial_{k} a_{0}$. The random couplings are assumed to be independent Gaussian-correlated quenched random variables with zero mean:

$$
\begin{aligned}
\left\langle m(\mathbf{x}) m\left(\mathbf{x}^{\prime}\right)\right\rangle_{\mathrm{dis}} & =g_{m} \delta^{(2)}\left(\mathbf{x}-\mathbf{x}^{\prime}\right), \\
\left\langle\tilde{a}_{0}(\mathbf{x}) \tilde{a}_{0}\left(\mathbf{x}^{\prime}\right)\right\rangle_{\mathrm{dis}} & =g_{0} \delta^{(2)}\left(\mathbf{x}-\mathbf{x}^{\prime}\right), \\
\left\langle\tilde{a}_{k}(\mathbf{x}) \tilde{a}_{k}\left(\mathbf{x}^{\prime}\right)\right\rangle_{\mathrm{dis}} & =g_{j} \delta^{(2)}\left(\mathbf{x}-\mathbf{x}^{\prime}\right), \quad k \in\{x, y\}, \\
\left\langle A_{0}(\mathbf{x}) A_{0}\left(\mathbf{x}^{\prime}\right)\right\rangle_{\mathrm{dis}} & =\Delta_{0} \delta^{(2)}\left(\mathbf{x}-\mathbf{x}^{\prime}\right), \\
\left\langle A_{k}(\mathbf{x}) A_{k}\left(\mathbf{x}^{\prime}\right)\right\rangle_{\mathrm{dis}} & =\Delta_{j} \delta^{(2)}\left(\mathbf{x}-\mathbf{x}^{\prime}\right), \quad k \in\{x, y\},
\end{aligned}
$$

where $\langle\cdot\rangle_{\text {dis }}$ indicates a disorder average and there is no sum over $k$. The disorder variances $g_{m}, g_{0}, g_{j}, \Delta_{0}, \Delta_{j}$ are positive constants.

We study the effects of the randomness in (2.29) using the replica trick, which enables the calculation of the disorderaveraged free energy and all observables that derive from it. To this end, we introduce $n_{r}$ replicas $\psi_{I, \ell}$ and $a_{\mu, \ell}$ with $\ell \in$ $\left\{1, \ldots, n_{r}\right\}$ and consider the replicated partition function,

$$
Z^{n_{r}}=\prod_{\ell}\left(\int \mathcal{D} \psi_{\ell} \mathcal{D} \bar{\psi}_{\ell} \mathcal{D} a_{\ell}\right) e^{i \sum_{\ell}\left(S^{(1)}\left[\psi_{\ell}, a_{\ell}\right]+S^{(2)}\left[a_{\ell}\right]+S_{\mathrm{dis}}\left[\psi_{\ell}, a_{\ell}\right]\right)},
$$

where $S^{(1)}\left[\psi_{\ell}, a_{\ell}\right]=\int d^{3} x \mathcal{L}^{(1)}\left(\psi_{\ell}, a_{\ell}\right)$ with $\mathcal{L}^{(1)}$ given in (2.1), $S^{(2)}\left[a_{\ell}\right]$ is given in (2.26), and $S_{\mathrm{dis}}\left[\psi_{\ell}, a_{\ell}\right]=$ $\int d^{3} x \mathcal{L}_{\text {dis }}\left(\psi_{\ell}, a_{\ell}\right)$ with $\mathcal{L}_{\text {dis }}$ given in (2.29). Using the identity

$$
\log Z=\lim _{n_{r} \rightarrow 0} \frac{Z^{n_{r}}-1}{n_{r}},
$$


the disorder-averaged free energy, proportional to $\langle\log Z\rangle_{\mathrm{dis}}$, is found upon disorder averaging. Using (2.30),

$$
\begin{aligned}
\left\langle Z^{n_{r}}\right\rangle_{\text {dis }}= & \prod_{\ell}\left(\int \mathcal{D} \psi_{\ell} \mathcal{D} \bar{\psi}_{\ell} \mathcal{D} a_{\ell}\right) \\
& \times e^{i \sum_{\ell}\left(S^{(1)}\left[\psi_{\ell}, a_{\ell}\right]+S^{(2)}\left[a_{\ell}\right]+i S^{(3)}\left[\psi_{\ell}, a_{\ell}\right]\right)},
\end{aligned}
$$

where

$$
\begin{aligned}
S^{(3)}\left[\psi_{\ell}, a_{\ell}\right]= & -\frac{1}{2} \sum_{k} \int d t d t^{\prime} d^{2} x\left[g_{m}\left(\bar{\psi}_{\ell} \psi_{\ell}\right)(t)\left(\bar{\psi}_{k} \psi_{k}\right)\left(t^{\prime}\right)\right. \\
& +g_{0}\left(\bar{\psi}_{\ell} \gamma^{0} \psi_{\ell}\right)(t)\left(\bar{\psi}_{k} \gamma^{0} \psi_{k}\right)\left(t^{\prime}\right) \\
& +g_{j}\left(\bar{\psi}_{\ell} \gamma^{j} \psi_{\ell}\right)(t)\left(\bar{\psi}_{k} \gamma^{j} \psi_{k}\right)\left(t^{\prime}\right) \\
& \left.+\Delta_{0} b_{\ell}(t) b_{k}\left(t^{\prime}\right)+\Delta_{j} \mathbf{e}_{\ell}(t) \cdot \mathbf{e}_{k}\left(t^{\prime}\right)\right],
\end{aligned}
$$

$\left(\bar{\psi}_{\ell}^{(I)} \psi_{\ell}^{(I)}\right)(t)\left(\bar{\psi}_{k}^{(J)} \psi_{k}^{(J)}\right)\left(t^{\prime}\right) \equiv \bar{\psi}_{\ell}^{(I)}(x, t) \psi_{\ell}^{(I)}(x, t) \bar{\psi}_{k}^{(J)}\left(x, t^{\prime}\right)$ $\psi_{k}^{(J)}\left(x, t^{\prime}\right)$, and similarly for the other terms appearing in $S_{E}^{(3)}$.

\section{RENORMALIZATION-GROUP ANALYSIS}

We now study the critical properties of the model introduced in Sec. II. Details of our calculations are presented in the Appendices.

\section{A. Large $N_{f}$ expansion and renormalization-group scheme}

The Euclidean effective action in $D+1$ dimensions is

$$
S_{E}=S_{E}^{(1)}+S_{E}^{(2)}+S_{E}^{(3)}
$$

where

$$
\begin{gathered}
S_{E}^{(1)}=\int d \tau d^{D} x\left\{\bar{\psi}_{\ell}^{(I)}\left[\gamma_{\tau}\left(\partial_{\tau}+i \frac{g}{\sqrt{N_{f}}} a_{\tau, \ell}\right)+v \gamma_{j}\left(\partial_{j}+i \frac{g}{\sqrt{N_{f}}} a_{j, \ell}\right)\right] \psi_{\ell}^{(I)}+m \bar{\psi}_{\ell}^{(I)} \psi_{\ell}^{(I)}+\frac{i \kappa}{2} a_{\ell} d a_{\ell}\right\}, \\
S_{E}^{(2)}=\int d \omega d^{D} k \frac{w_{x}}{2} a_{\ell, T}(\omega, k) \frac{k^{2}}{|k|+f(\omega, k)} a_{T, \ell}(-\omega,-k), \\
S_{E}^{(3)}=-\frac{1}{2} \int d \tau d \tau^{\prime} d^{D} x\left[g_{m}\left(\bar{\psi}_{\ell}^{(I)} \psi_{\ell}^{(I)}\right)(\tau)\left(\bar{\psi}_{k}^{(J)} \psi_{k}^{(J)}\right)\left(\tau^{\prime}\right)+g_{0}\left(\bar{\psi}_{\ell}^{(I)} \gamma^{0} \psi_{\ell}^{(I)}\right)(\tau)\left(\bar{\psi}_{k}^{(J)} \gamma^{0} \psi_{k}^{(J)}\right)\left(\tau^{\prime}\right)\right. \\
\left.+g_{j}\left(\bar{\psi}_{\ell}^{(I)} \gamma^{j} \psi_{\ell}^{(I)}\right)(\tau)\left(\bar{\psi}_{k}^{(J)} \gamma^{j} \psi_{k}^{(J)}\right)\left(\tau^{\prime}\right)+\Delta_{0} b_{\ell}(\tau) b_{k}\left(\tau^{\prime}\right)+\Delta_{j} \mathbf{e}_{\ell}(\tau) \cdot \mathbf{e}_{k}\left(\tau^{\prime}\right)\right] .
\end{gathered}
$$

Replica indices $\ell, k \in\left\{1, \ldots, n_{r}\right\}$ and flavor indices $I, J \in$ $\left\{1, \ldots, N_{f}\right\}$ with repeated indices summed. In Euclidean signature $(+,+,+)$, the coordinates $\left(\tau, x_{j}\right)=\left(i t, x_{j}\right)$, Fourier space variables $\left(\omega, k_{j}\right)=\left(-i k_{0}, k_{j}\right)$, and the $\gamma$ matrices $\left(\gamma_{0}, \gamma_{1}, \gamma_{2}\right)=\left(\sigma^{3}, \sigma^{1}, \sigma^{2}\right)$. We have set the longitudinal component of $a_{i}$ to zero (Coulomb gauge): $a_{i}(\omega, k)=$ $i \frac{k_{j}}{|k|} \epsilon_{j i} a_{T}(\omega, k)$. The gauge coupling is $g / \sqrt{N_{f}}$ with $g$ fixed and $N_{f} \rightarrow \infty$ [68], $v$ is the Dirac composite fermion velocity, and the Dirac mass $m$ vanishes at criticality. The disorder variances $g_{m}, g_{0}, g_{j}, \Delta_{0}$, and $\Delta_{j}$ are assumed to scale as $1 / N_{f}$. The Chern-Simons level is controlled by $\kappa=\frac{2 \theta-1}{4 \pi}$ : $\kappa=0$ gives an IQHT and $\kappa=1 / 4 \pi$ gives a SIT. $w_{x}=\frac{e_{*}^{2}}{4 \pi}$ parametrizes the strength of the dissipative Coulomb interaction and $f(\omega, k)=\frac{\sigma_{e} k^{2}}{|\omega|+D_{e} k^{2}}$. The nondynamical (electromagnetic) field $A_{\mu}=0$. We will often leave replica and flavor indices, as well as the spacetime dependence of fields, implicit.

We regularize UV divergent integrals that appear in our renormalization-group analysis of $S_{E}$ using dimensional reduction $[25,69,70]$. This is the standard approach (e.g., [25,70-73] and references therein) used in the study of theories of Chern-Simons gauge fields coupled to matter and, in contrast to dimensional regularization, has been shown to preserve gauge invariance at least to two-loop order in the perturbative analysis of $S_{E}^{(1)}$ [70]. We assume without proof that this regularization procedure maintains gauge invariance in our large $N_{f}$ study of $S_{E}$, which involves three-loop integrals. We consider a slight variation of the conventional dimensional reduction approach. There are two rules to follow. First, all vector, tensor, and spinor algebra is performed in three dimensions; in particular, the antisymmetric symbol $\epsilon^{\mu \nu \rho}$ obeys the usual 3D identities. Second, loop integrals are analytically continued to general (Euclidean) spatial dimension $D \leqslant 2$ :

$$
\int \frac{d \omega d^{2} k}{(2 \pi)^{3}} \rightarrow \mu^{\epsilon} \int \frac{d \omega d^{D} k}{(2 \pi)^{D+1}},
$$

where $\epsilon=2-D$ and $\mu$ is the renormalization-group scale. (Typically in dimensional reduction the spacetime dimension is analytically continued.) Simple poles proportional to $2 / \epsilon$ are identified with logarithmic divergences proportional to $\log \left(\Lambda^{2} / \mu^{2}\right)$ in a theory with momentum cutoff $\Lambda$; power-law divergences are set to zero.

The large $N_{f}$ Feynman rules that derive from $S_{E}$ at $m=0$ are given in Fig. 1. Note that we have summed once and for all the geometric series of fermion bubble diagrams in Fig. 2 to obtain the effective gauge field propagator:

$$
G_{m n}=\left(\begin{array}{cc}
\frac{g^{2}}{16} \frac{k^{2}}{\sqrt{\omega^{2}+v^{2} k^{2}}} & i \kappa|k| \\
i \kappa|k| & w_{x} \frac{k^{2}}{|k|+f(\omega, k)}+\frac{g^{2}}{16} \sqrt{\omega^{2}+v^{2} k^{2}}
\end{array}\right)_{m n}^{-1},
$$

where $m, n \in\{0, T\}$ correspond to the zeroth and transverse components of $a_{\mu}$. At large $N_{f}$ this resummation is equivalent to the random-phase approximation. The same effect also leads to a screening of the $g_{0}$ and $g_{j}$ disorders (see Appendix C) $[29,30]$. Aside from a few exceptions that we will discuss, we have found disorder screening to be a subleading effect in our analysis.

We use minimal subtraction [74,75] to renormalize $S_{E}$. In this scheme, simple poles in $\epsilon$ appear in counterterms $b_{\lambda_{a}}\left(\vec{\lambda}^{R}\right) / \epsilon$ that relate bare $(B)$ and renormalized $(R)$ couplings:

$$
\lambda_{a}^{B} \mu^{-\Delta_{a}(\epsilon)}=\lambda_{a}^{R}(\mu, \epsilon)+\frac{b_{\lambda_{a}}\left[\vec{\lambda}^{R}(\mu, \epsilon)\right]}{\epsilon},
$$




$$
\begin{aligned}
& \longrightarrow W^{G_{\mu \nu}} \\
& \longrightarrow=\frac{+i\left[\gamma_{0} k_{0}+v \gamma_{j} k_{j}\right]}{k_{0}+v^{2} k_{j}^{2}}
\end{aligned}
$$

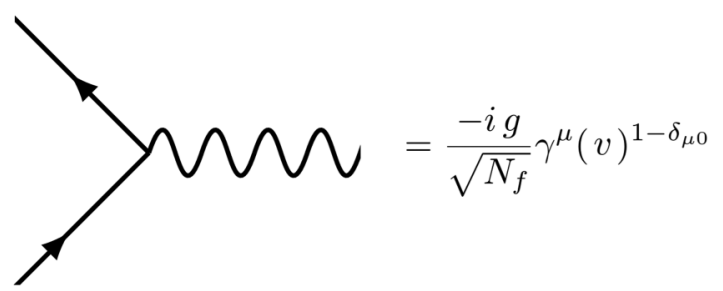

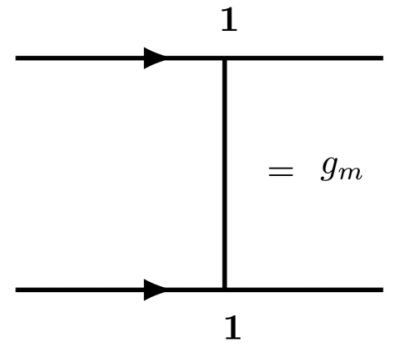

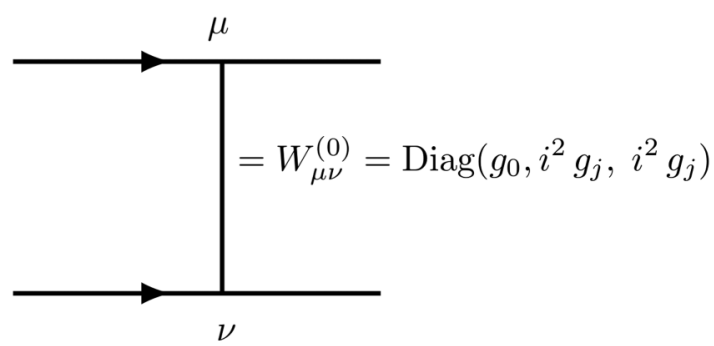

FIG. 1. Feynman rules of $S_{E}$. The wavy line denotes the effective gauge field propagator and the directed solid line indicates the fermion propagator with $m=0$. Disorder is represented by a solid line without an arrow and specified by its disorder variance $\left(g_{m}, g_{0}, g_{j}\right)$. Screenings of the disorder $\left(g_{0}, g_{j}\right)$ and topological disorder $\left(\Delta_{0}, \Delta_{j}\right)$ are discussed in Appendix C.

where the vector of coupling constants (either $B$ or $R$ )

$$
\vec{\lambda}=\left(\frac{g^{2}}{N_{f}}, v, m, \kappa, w_{x}, \sigma_{e}, D_{e}, g_{m}, g_{0}, g_{j}, \Delta_{0}, \Delta_{j}\right)^{T} .
$$

The renormalized couplings $\lambda_{a}^{R}(\mu, \epsilon)$ and residues $b_{\lambda_{a}}\left[\vec{\lambda}^{R}(\mu, \epsilon)\right]$ are analytic in $\epsilon$. The higher-order poles that generally occur on the right-hand side of Eq. (3.7) can be set to zero. The bare couplings $\lambda_{a}^{B}$ have engineering dimensions equal to $\Delta_{\lambda_{a}}(\epsilon)$ while the renormalized couplings are dimensionless. Appendix A details the calculation of the counterterms $b_{\lambda_{a}}\left(\vec{\lambda}^{R}\right) / \epsilon$. The engineering dimensions $\Delta_{\lambda_{a}}(\epsilon)$ of the bare couplings are given in Table II. These dimensions are determined as follows. Each term in $S_{E}^{(1)}$ is dimensionless with the assignments:

$$
\begin{gathered}
\Delta_{\tau}=-\Delta_{\omega}=-z, \quad \Delta_{x}=-\Delta_{k}=-1 \\
\Delta_{\psi(\tau, x)}=\frac{1}{2}-\frac{\epsilon}{2}, \quad \Delta_{a_{0}^{E}(\tau, x)}=z-\eta \epsilon, \quad \Delta_{a_{j}^{E}(\tau, x)}=1-\eta \epsilon
\end{gathered}
$$

and

$$
\Delta_{g^{2} / N_{f}}=2 \eta \epsilon, \quad \Delta_{v}=z-1, \quad \Delta_{m}=z, \quad \Delta_{\kappa}=(2 \eta-1) \epsilon,
$$

where $\eta$ is an arbitrary constant. We have introduced the dynamical critical exponent $z$ with a value to be determined later; in the absence of $S_{E}^{(2)}$ and $S_{E}^{(3)}$, relativistic symmetry requires that $v$ be dimensionless and $z=1$. In the large $N_{f}$ expansion, $g$ is fixed and we formally take $\Delta_{N_{f}}=-2 \eta \epsilon$. The effective gauge field propagator is consistent with the engineering dimensions $\Delta_{a_{0}^{E}(\omega, k)}=-D-\eta \epsilon$ and $\Delta_{a_{j}^{E}(\omega, k)}=(1-$ $z)-D-\eta \epsilon$ if $\eta=1 / 2$. The dimensions of the remaining couplings ensure the terms in $S_{E}^{(2)}$ and $S_{E}^{(3)}$ are dimensionless.

The beta functions $\beta_{\lambda_{a}}$ at $\epsilon=0$ are read off from the residues $b_{\lambda_{a}}\left(\vec{\lambda}^{R}\right)$ using

$$
\begin{aligned}
\beta_{\lambda_{a}}\left(\vec{\lambda}^{R}\right) \equiv & -\mu \frac{\partial \lambda_{a}^{R}}{\partial \mu}=\bar{\Delta}_{\lambda_{a}} \lambda_{a}^{R}+\rho_{\lambda_{a}} b_{\lambda_{a}}\left(\vec{\lambda}^{R}\right) \\
& -\sum_{c} \rho_{\lambda_{c}} \lambda_{c}^{R} \frac{\partial b_{\lambda_{a}}\left(\vec{\lambda}^{R}\right)}{\partial \lambda_{c}^{R}} .
\end{aligned}
$$

There is no sum over $a$ in Eq. (3.12). The minus sign in front of $\mu \frac{\partial \lambda_{a}^{R}}{\partial \mu}$ means that a relevant/irrelevant coupling has a positive/negative beta function. Notice that only $g^{2} / N_{f}$ and the variances $g_{m}, g_{0}, g_{j}$ can contribute to the derivative term on the right-hand side of Eq. (3.12).

We characterize any fixed points $\beta_{\lambda_{a}}\left[\left(\vec{\lambda}^{R}\right)^{*}\right]=0$ by the dynamical critical exponent $z$ and correlation length exponent $v$, evaluated at the fixed point. The dynamical critical exponent enters the beta functions (3.12) via $\bar{\Delta}_{\lambda_{a}}$ (see Table II) and we determine its value by the condition of vanishing velocity beta

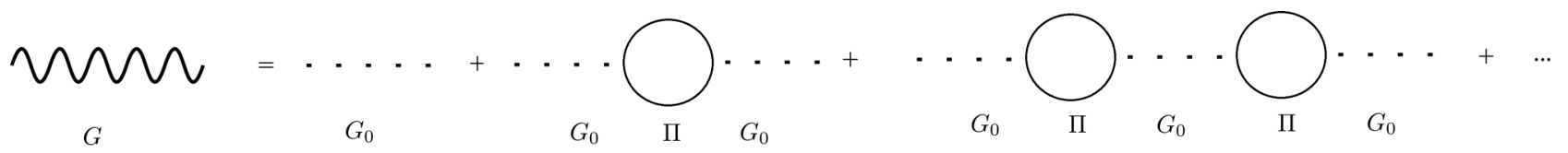

FIG. 2. The effective gauge field propagator $G$. The dotted line $G_{0}$ represents the bare gauge field propagator and $\Pi_{\mu \nu}$ is the one-loop gauge field self-energy. Each term in this geometric series of diagrams produces an $\mathcal{O}\left(N_{f}^{0}\right)$ correction to the gauge field propagator, since each fermion loop contributes a factor of $N_{f}$ and the two vertices associated to each loop contribute an additional factor of $g^{2} / N_{f}$. 
TABLE II. Engineering dimension $\Delta_{\lambda_{a}}(\epsilon)=\bar{\Delta}_{\lambda_{a}}+\rho_{\lambda_{a}} \epsilon$ of bare coupling $\lambda_{a}^{B}$ ( $B$ superscript omitted in the table), where $\bar{\Delta}_{\lambda_{a}}$ is independent of $\epsilon=2-D$ and $\rho_{\lambda_{a}}$ is the constant coefficient of $\epsilon$.

\begin{tabular}{lccc}
\hline \hline$\lambda_{a}$ & $\Delta_{\lambda_{a}}(\epsilon)$ & $\bar{\Delta}_{\lambda_{a}}$ & $\rho_{\lambda_{a}}$ \\
\hline$\overline{g^{2}}$ & $\epsilon$ & 0 & 1 \\
$\left\{v, w_{x}, \sigma_{e}\right\}$ & $z-1$ & $z-1$ & 0 \\
$m$ & $z$ & $z$ & 0 \\
$\left\{\kappa, \Delta_{j}\right\}$ & 0 & 0 & 0 \\
$D_{e}$ & $z-2$ & $z-2$ & 0 \\
$\left\{g_{m}, g_{0}, g_{j}\right\}$ & $2 z-D$ & $2 z-2$ & 1 \\
$\Delta_{0}$ & $2 z-2$ & $2 z-2$ & 0 \\
\hline \hline
\end{tabular}

function $\beta_{v}\left(\vec{\lambda}^{R}\right)=0$ :

$$
z=1+\frac{1}{v^{R}} \sum_{c} \rho_{\lambda_{c}} \lambda_{c}^{R} \frac{\partial b_{v}\left[\left(\vec{\lambda}^{R}\right)^{*}\right]}{\partial \lambda_{c}^{R}}
$$

Nonzero $\beta_{v}$ implies a quantum correction to the tree-level dynamical exponent, i.e., the engineering dimension $-\Delta_{\tau}$. This follows from the fermion dispersion relation $|\omega|=v|k|$, e.g., [48]. We have chosen to introduce an arbitrary $z$ in Table II with a value to be determined by vanishing renormalization of the velocity. An equivalent choice is to take engineering dimensions consistent with conformal invariance and infer any correction to the tree-level dynamical scaling from a nonzero velocity beta function.

Since the transitions we consider in this paper are tuned by the Dirac mass, we define the correlation length $\xi$ as the inverse momentum scale $\mu_{0}^{-1}$ at which $v^{R}\left(\mu_{0}\right) / m^{R}\left(\mu_{0}\right)=1$. [The factor of $v^{R}\left(\mu_{0}\right)$ accounts for possible running of the velocity in the equivalent approach where $z=1$ is chosen in Table II and the nonzero velocity beta function determines the correction $z-1$ to dynamical scaling.] We write the mass beta function as

$$
\beta_{m}\left(\vec{\lambda}^{R}\right)=\left[z-\gamma_{\bar{\psi} \psi}\left(\vec{\lambda}^{R}\right)\right] m^{R},
$$

where the anomalous dimension $\gamma_{\bar{\psi} \psi}$ controls the asymptotic scaling of the correlation function $\langle\bar{\psi} \psi(\tau, x) \bar{\psi} \psi(0)\rangle \sim$ $\left|v^{2} \tau^{2}+x^{2}\right|^{-\left(D+\gamma_{\bar{\psi}}\right)}$. Using Eqs. (3.12)-(3.14), we find

$$
\xi=\Lambda^{-1}\left(\frac{m_{\Lambda} / v_{\Lambda}}{\Lambda}\right)^{-v}
$$

where $\Lambda$ is an arbitrary momentum cutoff defining the "initial conditions," $m^{R}(\Lambda)=m_{\Lambda} / \Lambda^{z}$ and $v^{R}(\Lambda)=v_{\Lambda} / \Lambda^{z-1}$, and the inverse correlation length exponent

$$
v^{-1}=z-\gamma_{\bar{\psi} \psi} .
$$

Note that $m^{R}$ does not enter the residues $b_{\lambda_{a}}\left(\vec{\lambda}^{R}\right)$ with $\lambda_{a}^{R} \neq m^{R}$ and only appears linearly in $b_{m}\left(\vec{\lambda}^{R}\right)$.

In the remainder of the main text, we drop the $B$ and $R$ superscripts for notional simplicity; the couplings appearing in the beta functions are renormalized ones.

\section{B. General beta functions}

We now present the results of our renormalization-group calculation, which is valid to order $1 / N_{f}$ in the large $N_{f}$ expansion. See the Appendices for details.

A vanishing velocity beta function determines the dynamical critical exponent to be

$$
z=1+\overline{g_{m}}+\overline{g_{0}}+2 \overline{g_{j}}-F_{w}\left(\overline{w_{x}}, \kappa, \overline{\sigma_{e}}\right)
$$

where $g_{1}=g^{2} / 16$, the rescaled couplings are

$$
\begin{aligned}
& \bar{m}=\frac{m}{v}, \quad \overline{w_{x}}=\frac{w_{x}}{v}, \quad \overline{\sigma_{e}}=\frac{\sigma_{e}}{v}, \quad \overline{g_{m}}=\frac{g_{m}}{2 \pi v^{2}}, \\
& \overline{g_{0}}=\frac{g_{0}}{2 \pi v^{2}}, \quad \overline{g_{j}}=\frac{g_{j}}{2 \pi v^{2}}, \quad \overline{\Delta_{0}}=\Delta_{0}, \quad \overline{\Delta_{j}}=\Delta_{j} v^{2},
\end{aligned}
$$

and

$$
F_{w}\left(\overline{w_{x}}, \kappa, \overline{\sigma_{e}}\right)=\frac{1}{4 \pi^{2} N_{f}} \int_{-\infty}^{\infty} d y \frac{g_{1}\left(-1+2 y^{2}\right)\left(\sigma_{e}+|y|\right)+\overline{w_{x}}|y| \sqrt{1+y^{2}}}{\left(1+y^{2}\right)^{2}\left[\sqrt{1+y^{2}}\left(g_{1}^{2}+\kappa^{2}\right)\left(\overline{\sigma_{e}}+|y|\right)+g_{1} \overline{w_{x}}|y|\right]} .
$$

The beta functions $\beta_{\lambda_{a}}=-\mu \frac{\partial \lambda_{a}}{\partial \mu}$ for the remaining couplings take the form

$$
\begin{gathered}
\beta_{\overline{w_{x}}}=\overline{w_{x}}(z-1), \\
\beta_{\overline{\sigma_{e}}}=\overline{\sigma_{e}}(z-1), \\
\beta_{D_{e}}=D_{e}(z-2), \\
\beta_{\bar{m}}=\bar{m}\left(z-\gamma_{\bar{\psi} \psi}\right), \\
\beta_{\overline{g_{m}}}=2 \overline{g_{m}}\left(z-1+\frac{2 \overline{g_{0}} \overline{g_{j}}}{\overline{g_{m}}}-\gamma_{\bar{\psi} \psi}\right), \\
\beta_{\overline{g_{0}}}=2 \overline{g_{0}}\left(z-1+\frac{2 \overline{g_{j}}}{\overline{g_{m}}}\right), \\
\beta_{\overline{g_{j}}}=2 \overline{g_{j}}\left(z-1+\frac{\overline{g_{m}} \overline{g_{0}}}{\overline{g_{j}}}-\overline{g_{m}}-\overline{g_{0}}-2 \overline{g_{j}}+F_{w}\left(\overline{w_{x}}, \kappa, \overline{\sigma_{e}}\right)\right),
\end{gathered}
$$




$$
\begin{gathered}
\beta_{\overline{\Delta_{0}}}=\overline{\Delta_{0}}(2 z-2)+\frac{\overline{g_{m}}\left[\overline{\Delta_{j}}\left(g_{1}+\overline{w_{x}}\right)^{2}+\overline{\Delta_{0}} \kappa^{2}\right]}{64\left[g_{1}\left(g_{1}+\overline{w_{x}}\right)\right]^{2}}+\frac{\overline{g_{0}} \overline{g_{m}} N_{f} \pi v^{2}}{32}, \\
\beta_{\overline{\Delta_{j}}}=\frac{\overline{g_{m}}\left(g_{1}^{2} \overline{\Delta_{0}}+\kappa^{2} \overline{\Delta_{j}}\right)}{128\left[g_{1}\left(g_{1}+\overline{w_{x}}\right)+\kappa^{2}\right]^{2}}+\frac{\overline{g_{j}} \overline{g_{m}} N_{f} \pi v^{4}}{64},
\end{gathered}
$$

where $z$ is given in Eq. (3.17), the mass anomalous dimension

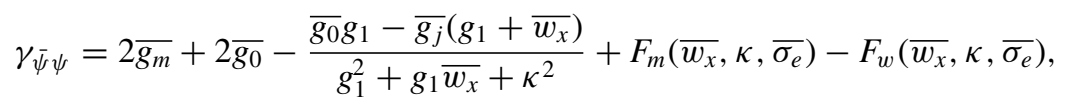

and

$$
\begin{aligned}
F_{m}\left(\overline{w_{x}}, \kappa, \overline{\sigma_{e}}\right)= & \frac{1}{4 \pi^{2} N_{f}} \int_{-\infty}^{\infty} d y\left[\frac{g_{1}\left(\overline{\sigma_{e}}+|y|\right)\left(-2 y^{2}-3\right)-\overline{w_{x}} \sqrt{1+y^{2}}|y|}{\left(1+y^{2}\right)^{2}\left[\sqrt{1+y^{2}}\left(g_{1}^{2}+\kappa^{2}\right)\left(\overline{\sigma_{e}}+|y|\right)+g_{1} \overline{w_{x}}|y|\right]}\right. \\
& +\frac{\left(\overline{\sigma_{e}}+|y|\right)\left[\sqrt{1+y^{2}}\left(g_{1}^{2}-\kappa^{2}\right)\left(\overline{\sigma_{e}}+|y|\right)+g_{1} \overline{w_{x}}|y|\right]}{\left.2\left(1+y^{2}\right)\left[\sqrt{1+y^{2}}\left(g_{1}^{2}+\kappa^{2}\right)\left(\overline{\sigma_{e}}+|y|\right)+g_{1} \overline{w_{x}}|y|\right]^{2}\right]} .
\end{aligned}
$$

To simplify the above expressions, we have ignored terms that arise from the screening of the $g_{0}$ and $g_{j}$ disorders (see Appendix C); our detailed analysis below includes such effects whenever relevant. The gauge coupling $g / \sqrt{N_{f}}$ is marginal once the large $N_{f}$ effective gauge field propagator in Fig. 2 is adopted and so its beta function is not included.

Let us make a few additional comments about these expressions.

(1) In general, the above beta functions do not have an IR stable solution at $\bar{m}=0$, even when disorder screening is included. In the remaining sections, we analyze cases for which we have found fixed points when a symmetry is present.

(2) We have taken the variances to scale as $1 / N_{f}$ for $N_{f} \rightarrow \infty$. The beta functions have terms that scale as $1 / N_{f}$ and $1 / N_{f}^{2}$. The "classical" contributions to the beta functions arising from the engineering dimensions of couplings scale as $1 / N_{f}$; the "quantum" corrections generally scale as $1 / N_{f}^{2}$. The exception to the latter appears in the third term in $\beta_{\overline{\Delta_{0}}}$ and the second term in $\beta_{\overline{\Delta_{j}}}$. A possible alternative scaling scheme allows the $\overline{\Delta_{0}}$ and $\overline{\Delta_{j}}$ to scale as $N^{0}$ with all other variances scaling as $1 / N$.

(3) The first three beta functions $\beta_{\overline{\bar{w}_{x}}}, \beta_{\overline{\sigma_{e}}}$, and $\beta_{D_{e}}$ characterize the dissipative Coulomb interaction. In our analysis, we consider $z<2$ and so the diffusion constant $D_{e}$ is an irrelevant parameter that will be set to zero. A nonzero Coulomb interaction allows for two classes of fixed points: (1) a finite Coulomb interaction either with $\overline{w_{x}}, \overline{\sigma_{e}} \neq 0$ and $z=1$ or with $\overline{w_{x}}=\overline{\sigma_{e}}=0$ and $z$ determined by Eq. (3.17) and (2) an infinite Coulomb interaction with $\overline{w_{x}} \rightarrow \infty, \overline{\sigma_{e}} \rightarrow \infty$, and $1<z<2$ that is controlled by the dissipation parameter $\overline{\sigma_{e}} / \overline{w_{x}}$.

(4) Whenever two of the three disorder variances $g_{m}, g_{0}$, and $g_{j}$ are considered, the third variance is radiatively generated. When all three variances $g_{m}, g_{0}$, and $g_{j}$ are present, both types of topological disorder $\Delta_{0}$ and $\Delta_{j}$ are generated. This is consistent with the symmetry assignments in Table I.

\section{Finite Coulomb interaction}

\section{No disorder}

In the absence of any disorder, the only nontrivial beta functions are associated to the Coulomb interaction:

$$
\beta_{\overline{w_{x}}}=-\overline{w_{x}} F_{w}\left(\overline{w_{x}}, \kappa, \overline{\sigma_{e}}\right) .
$$

Since $\frac{1}{\overline{\sigma_{e}}} \beta_{\overline{\sigma_{e}}}=\frac{1}{\bar{w}_{x}} \beta_{\overline{w_{x}}}$, it is sufficient to consider the behavior of $\frac{\sigma_{e}}{w_{x}}$ when studying a finite Coulomb interaction. The integral that defines $F_{w}$ in Eq. (3.19) can only be evaluated numerically for general $\overline{\sigma_{e}}$. We have found that $F_{w}$ is positive for any $\kappa$ when $\overline{w_{x}} \neq 0$. Consequently, the clean fixed point with ${\overline{w_{x}}}^{*}={\overline{\sigma_{e}}}^{*}=0$ is perturbatively stable to the addition of a Coulomb interaction and $z=1$. Two examples for the behavior of $\beta_{\overline{w_{x}}}$ are displayed in Fig. 3 .

In particular, in the limit of $\overline{\sigma_{e}}=0$ the beta function for $\overline{w_{x}}$ is always negative for any Chern-Simons coupling $\kappa$. This result should be contrasted with earlier work [28] where a critical value of $|\kappa|$ was reported above which the Coulomb interaction was found to be relevant. The discrepancy seems to arise from assigning the Chern-Simons coupling $\kappa$ an engineering dimension proportional to $\epsilon=2-D$. This choice, which appears to be inconsistent with the scaling of the effective gauge field propagator in the large $N_{f}$ expansion, results in additional derivatives with respect to $\kappa$ in the beta function in (3.12). As a check on our calculation, we find the mass anomalous dimension is given by

$$
\gamma_{\bar{\psi} \psi}=\frac{128}{3 \pi^{2} N_{f}} \frac{1-512 \kappa^{2}}{\left(1+256 \kappa^{2}\right)^{2}}
$$

in agreement with $[25,67,76,77]$ for general $\kappa$. For the IQHT $(\kappa=0), v^{-1} \approx 1-4.3 / N_{f} ;$ for the SIT $(\kappa=1 / 4 \pi), v^{-1} \approx$ $1+1.4 / N_{f}$.

\section{2. $\mathcal{C}$ symmetry}

According to Table I, the Coulomb couplings and random mass disorder $\left(g_{m}\right)$ are allowed when there is chargeconjugation symmetry. The beta functions are

$$
\begin{gathered}
\beta_{\overline{w_{x}}}=\overline{w_{x}}\left[\overline{g_{m}}-F_{w}\left(\overline{w_{x}}, \kappa, \overline{\sigma_{e}}\right)\right], \\
\beta_{\overline{g_{m}}}=-2 \overline{g_{m}}\left[\overline{g_{m}}+F_{m}\left(\overline{w_{x}}, \kappa, \overline{\sigma_{e}}\right)\right],
\end{gathered}
$$

where $F_{w}$ and $F_{m}$ are defined in Eqs. (3.19) and (3.30). The flow of the random mass is controlled by the mass 


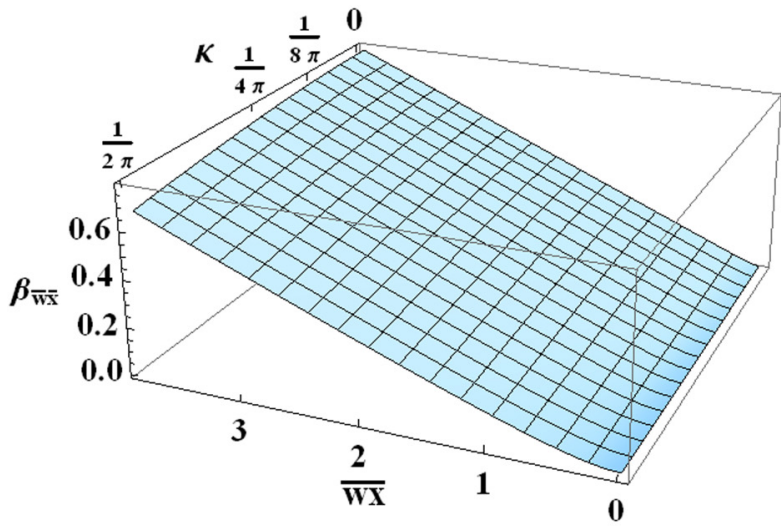

(a)

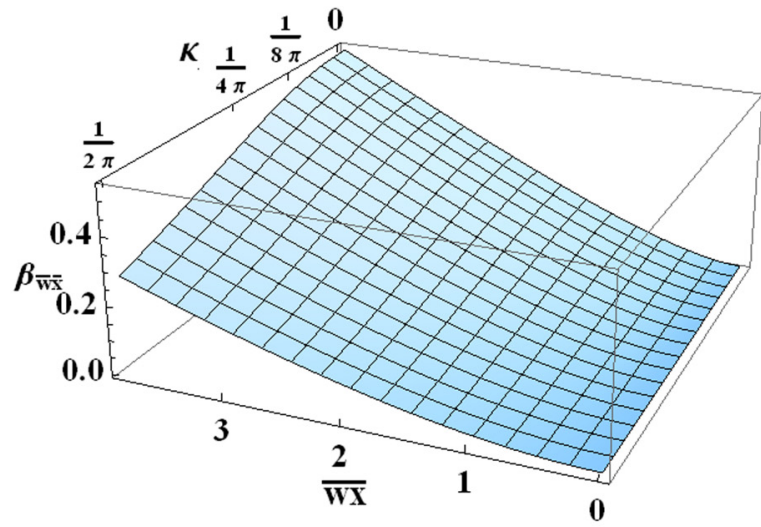

(b)

FIG. 3. $\beta_{\overline{w_{x}}}$ as a function of $\overline{w_{x}}$ and $\kappa$. (a) $\overline{\sigma_{e}}=0$. (b) $\overline{\sigma_{e}}=5.0$.

anomalous dimension $\gamma_{\bar{\psi} \psi}=2 \overline{g_{m}}-F_{w}\left(\overline{w_{x}}, \kappa, \overline{\sigma_{e}}\right)+$ $F_{m}\left(\overline{w_{x}}, \kappa, \overline{\sigma_{e}}\right)$. Within the large $N_{f}$ approximation, random mass disorder is only a relevant perturbation to the clean fixed point of the previous section $\left(\bar{w}_{x}^{*}=\bar{\sigma}_{e}{ }^{*}=0\right)$ when $\gamma_{\bar{\psi} \psi}<0$, i.e., when $1<512 \kappa^{2}$ [see Eq. (3.32)], in agreement with [31]. The presence of a Coulomb interaction does not appear to alter this conclusion within our analysis. For $\kappa=1 / 4 \pi$ (or any $\kappa^{2}>1 / 512$ ), there exists a line of fixed points with finite disorder and Coulomb interaction parametrized by $\overline{\sigma_{e}}$. Figure 4 shows a few examples of this behavior. Since $\beta_{\overline{w_{x}}} \propto z-1$ and $\beta_{\overline{g_{m}}} \propto-\gamma_{\bar{\psi} \psi}$ when $z=1$, any fixed point with finite disorder and Coulomb interaction has

$$
v^{-1}=z=1 \text {. }
$$

At the (generally unstable) fixed point with nonzero random mass disorder and vanishing Coulomb interaction,

$$
v^{-1}=1 \quad \text { and } \quad z=1-\frac{128}{3 \pi^{2} N_{f}} \frac{1-512 \kappa^{2}}{\left(1+256 \kappa^{2}\right)^{2}},
$$

where $\kappa^{2}>1 / 512$. For $\kappa=1 / 4 \pi, z \approx 1+1.4 / N_{f}$.

It is interesting to compare our results for the critical exponents with recent analytic and numerical studies of the dirty XY model. In a large $N_{f}$ expansion [38] report $v=1$ and $z=1+0.5 / N_{f}$; numerics [42] directly probes $N_{f}=1$ with the result $v=1.16(5)$ and $z=1.52(3)$. In [30], a finite disorder fixed point of quantum electrodynamics without the Chern-Simons term was found using an $\epsilon$ expansion about $(3+1)$ D. Since our approximation schemes are different, there is no contradiction with our conclusion that random mass disorder is irrelevant when $\kappa=0$. Nevertheless, it would be interesting to consider this issue further.

\section{3. $\mathcal{C} \mathcal{T}$ symmetry}

According to Table I, the Coulomb coupling, random scalar potential $g_{0}$, and topological disorder $\Delta_{j}$ are allowed by $\mathcal{C} \mathcal{T}$ symmetry. Because a nonzero Chern-Simons term is odd under time-reversal symmetry, we only consider $\kappa=0$ in the next two subsections that study $\mathcal{C} \mathcal{T}$ and $\mathcal{T}$ preserving disorder. The beta functions are

$$
\begin{gathered}
\beta_{\overline{w_{x}}}=\overline{w_{x}}\left[\overline{g_{0}}\left(1-\phi_{1}\right)-F_{w}\left(\overline{w_{x}}, \kappa=0, \overline{\sigma_{e}}\right)\right], \\
\beta_{\overline{g_{0}}}=\frac{2 \overline{g_{0}}}{\overline{w_{x}}} \beta_{\overline{w_{x}}}, \\
\beta_{\overline{\Delta_{j}}}=0,
\end{gathered}
$$

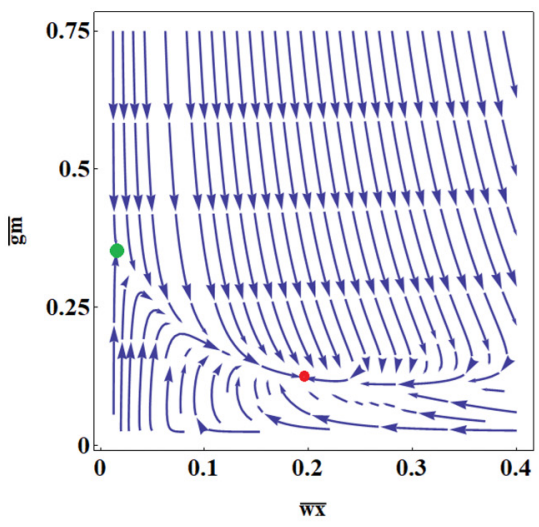

(a)

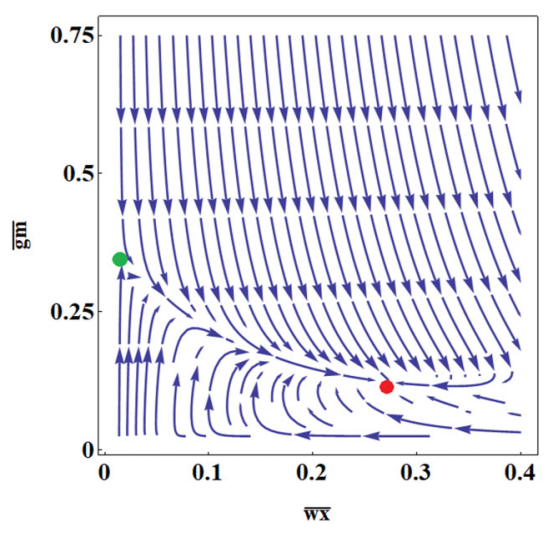

(b)

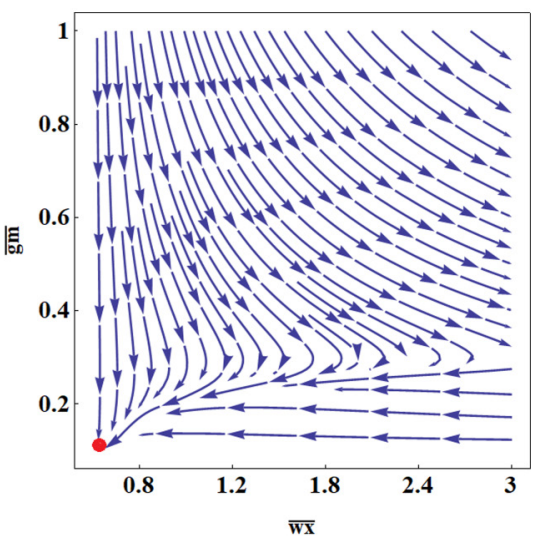

(c)

FIG. 4. RG flow of $\overline{w_{x}}$ vs $\overline{g_{m}}$. In the above plot, we choose $N_{f}=4$. (a) $\kappa=\frac{1}{4 \pi}, \overline{\sigma_{e}}=0$. (b) $\kappa=\frac{1}{4 \pi}, \overline{\sigma_{e}}=0.1$. (c) $\kappa=0, \overline{\sigma_{e}}=0$. 


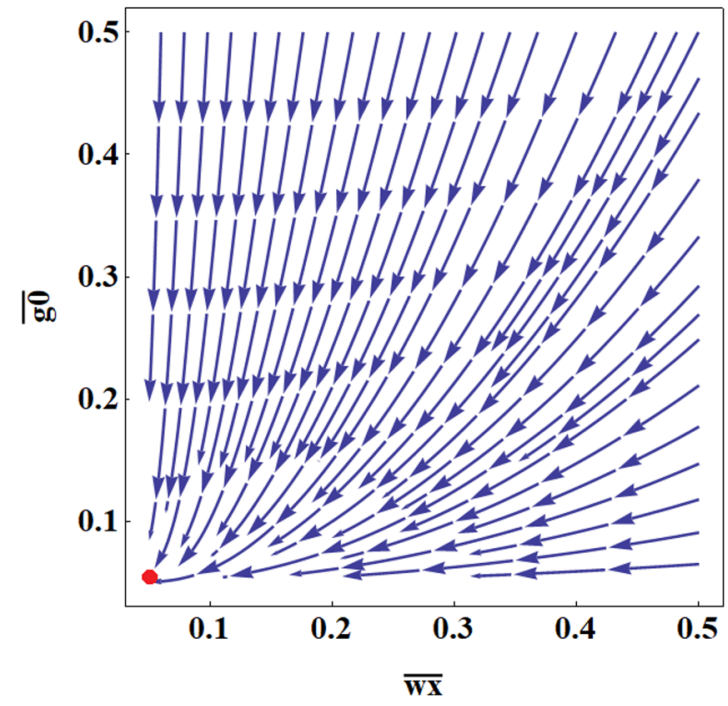

(a)

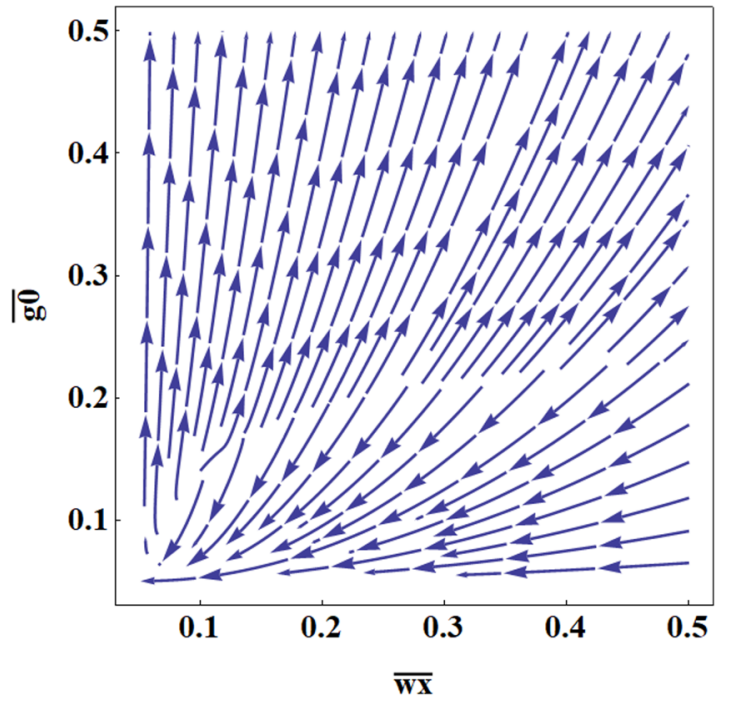

(b)

FIG. 5. RG flow of $\overline{w_{x}}$ vs $\overline{g_{0}}$. In the above figures, we set $N_{f}=4, \kappa=0, \overline{\sigma_{e}}=0$. (a) Disorder screening $\left(\phi_{1}=1\right)$. (b) No disorder screening $\left(\phi_{1}=0\right)$.

where $\phi_{1}$ isolates any terms that arise from the screening of the disorder: $\phi_{1}=0$ means disorder screening is ignored; $\phi_{1}=1$ means that disorder screening is included. While we are unaware of a general reason to exclude disorder screening, we will discuss the behavior of the above beta functions both with and without screening to illustrate its effect.

As mentioned previously, $F_{w}$ in Eq. (3.19) is positive for any $\kappa$ when $\overline{w_{x}}$ is nonzero; in particular, when $\overline{\sigma_{e}}=$ $0, F_{w}\left(\bar{w}_{x}, 0,0\right)$ is a monotonically increasing function that approaches $\frac{8}{N_{f} \pi^{2}}$ for $\overline{w_{x}} \rightarrow \infty$. When disorder screening is ignored $\left(\phi_{1}=0\right)$, there is a fixed surface defined by $\overline{g_{0}}=F_{w}$, which is parametrized by $\left(\bar{w}_{x}^{*}, \bar{g}_{0}^{*}, \bar{\Delta}_{j}^{*}\right)$, in agreement with [31]. This fixed surface is unstable, e.g., consider perturbation to $\overline{g_{0}}$ at fixed $\bar{w}_{x}^{*}$ and ${\overline{\Delta_{j}}}^{*}$. When disorder screening is included $\left(\phi_{1}=1\right)$, there is a line of stable fixed points parametrized by $\left(\bar{w}_{x}^{*}, \bar{g}_{0}^{*},{\overline{\Delta_{j}}}^{*}\right)=\left(0,0,{\overline{\Delta_{j}}}^{*}\right)$. This result is consistent with [30]. This behavior is illustrated in Fig. 5. The corresponding critical exponents at the stable fixed point $\left(\phi_{1}=1\right)$ reduce to those of the clean theory without Coulomb interactions. Recall that $g_{0}$ and $\Delta_{j}$ disorders are generated by random electrical vector potential $A_{j}(\mathbf{x})$ in the free Dirac fermion dual at $N_{f}=1$ for which a line of diffusive fixed points was found in [16]. It is unclear to what extent the line of fixed points parametrized by $\Delta_{j}$ is related.

If $\mathcal{C} \mathcal{T}$ and $\mathcal{T}$ are emergent symmetries of the SIT theory we consider, then the beta functions should only have fixedpoint solutions respecting these symmetries at $\kappa=1 / 4 \pi$. Unfortunately, the leading terms in the large $N_{f}$ beta functions do not produce any such nontrivial fixed points. Even if $\overline{g_{m}}$ is initially tuned to zero, the random mass beta function receives a positive correction from disorder screening equal to

$$
\delta \beta_{\overline{g_{m}}}=\frac{8 \bar{g}_{0}^{2} g_{1}^{2} \kappa^{4}}{\left(g_{1}^{2}+g_{1} \overline{w_{x}}+\kappa^{2}\right)^{4}} .
$$

Nonzero $\overline{g_{0}}$ and $\overline{g_{m}}$ then result in the generation of all couplings, for which we find runaway flow.

\section{4. $\mathcal{T}$ symmetry}

According to Table I, the Coulomb coupling, random vector potential $g_{j}$, and topological disorder $\Delta_{0}$ are allowed by $\mathcal{T}$ symmetry. The beta functions are

$$
\begin{gathered}
\beta_{\overline{w_{x}}}=\overline{w_{x}}\left\{2 \overline{g_{j}}\left[1-\phi_{1} \frac{g_{1}\left(g_{1}+2 \overline{w_{x}}\right)}{\left(g_{1}+\overline{w_{x}}\right)^{2}}\right]\right. \\
\left.-F_{w}\left(\overline{w_{x}}, \kappa=0, \overline{\sigma_{e}}\right)\right\}, \\
\beta_{\overline{g_{j}}}=0, \\
\beta_{\overline{\Delta_{0}}}=\frac{2 \overline{\Delta_{0}}}{\overline{w_{x}}} \beta_{\overline{w_{x}}},
\end{gathered}
$$

where we continue to use $\phi_{1}$ to isolate terms that arise from the screening of the disorder.

If screening is ignored $\left(\phi_{1}=0\right)$, there is a surface of fixed points defined by $2{\overline{g_{j}}}^{*}=F_{w}\left({\overline{w_{x}}}^{*}, 0,{\overline{\sigma_{e}}}^{*}\right)$ and parametrized by $\left({\overline{w_{x}}}^{*},{\overline{g_{j}}}^{*},{\overline{\Delta_{0}}}^{*}\right)$ with ${\overline{g_{j}}}^{*}<4 / N_{f} \pi^{2}$ and non-negative ${\overline{\Delta_{0}}}^{*}$. On this surface $z=1$ and $v^{-1}=1+2{\overline{g_{j}}}^{*}-\frac{{\overline{g_{j}}}^{*}}{g_{1}}-$ $F_{m}\left({\overline{w_{x}}}^{*}, 0,{\overline{\sigma_{e}}}^{*}\right) ; F_{m}$ is a monotonically decreasing function of $\bar{w}_{x}{ }^{*}$ when $\kappa=\bar{\sigma}_{e}{ }^{*}=0: \frac{128}{3 \pi^{2} N_{f}} \geqslant F_{m}\left({\overline{w_{x}}}^{*}\right) \geqslant-\frac{8}{\pi^{2} N_{f}}$. For fixed $\bar{g}_{j}{ }^{*}$, this surface is stable to small deformation by $\overline{w_{x}}$ since $F_{w}$ is an increasing function of $\overline{w_{x}}$. For $\overline{g_{j}}>4 / N_{f} \pi^{2}$, we find runaway flows. This behavior is shown in Fig. 6. If screening is included $\left(\phi_{1}=1\right)$, the fixed points are determined by the equation

$$
{\overline{g_{j}}}^{*}=\frac{F_{w}\left({\overline{w_{x}}}^{*}\right)}{2\left[1-\phi_{1} \frac{g_{1}\left(g_{1}+2 \bar{w}^{*}\right)}{\left(g_{1}+{\overline{w_{x}}}^{*}\right)^{2}}\right]} \equiv f_{s}\left({\overline{w_{x}}}^{*}\right)
$$

where $f_{s}\left({\overline{w_{x}}}^{*}\right)$ monotonically decreases from $f_{s}\left({\overline{w_{x}}}^{*}=0\right)=$ $\infty$ to $f_{s}\left({\overline{w_{x}}}^{*} \rightarrow \infty\right)=\frac{4}{N_{f} \pi^{2}}$. If $\bar{g}_{j}^{*}$ is chosen to be smaller than $\frac{4}{N_{f} \pi^{2}}$, then the RG flows to $\left({\overline{w_{x}}}^{*},{\overline{\Delta_{0}}}^{*}\right)=(0,0)$ because $\beta_{\overline{w_{x}}}$ is non-negative and only vanishes when $\overline{w_{x}}=0$. If 


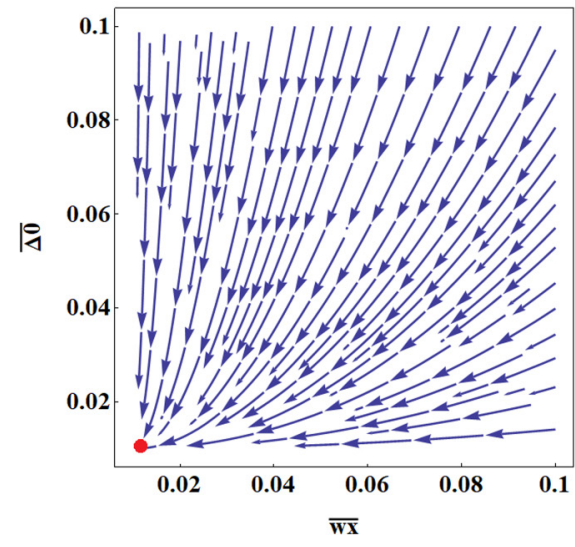

(a)

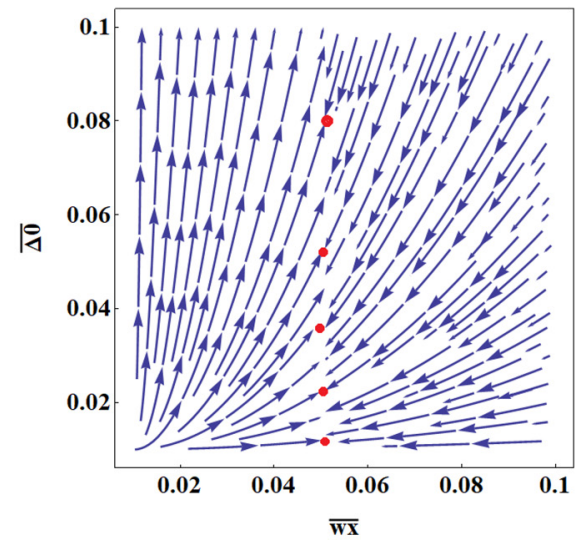

(b)

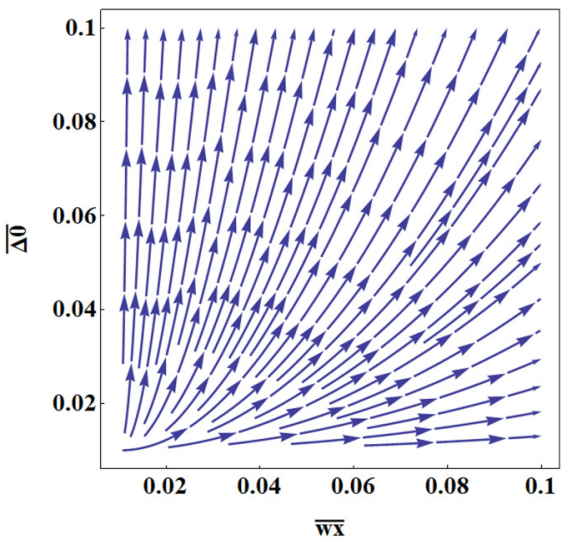

(c)

FIG. 6. RG flow of $\overline{w_{x}}$ vs $\overline{\Delta_{0}}$. In the above figures, we take the nonscreening choice $\left(\phi_{1}=0\right)$ with $\kappa=\sigma_{e}=0, N_{f}=4$, so the critical value $\frac{4}{N_{f} \pi^{2}}=0.101$. (a) $\overline{g_{j}}=0$, flowing to a clean fixed point. (b) $\overline{g_{j}}=0.04<\frac{4}{N_{f} \pi^{2}}$, there is a fixed line. (c) $\overline{g_{j}}=0.2>\frac{4}{N_{f} \pi^{2}}$, running to strong-coupled.

${\overline{g_{j}}}^{*}>\frac{4}{N_{f} \pi^{2}}$, then there exists a finite value of $\bar{w}_{x}^{*}$ for which the beta function vanishes; however, the resulting fixed point is IR unstable.

\section{Infinite Coulomb interaction}

Dissipation has played only a minor role in the above analysis. We will now discuss how dissipation allows for fixed points with $z \neq 1$ in the presence of a Coulomb interaction [48].

The runnings of the Coulomb interaction parameters $\overline{w_{x}}$ and $\overline{\sigma_{e}}$ are determined by their engineering dimensions, which are both equal to $z-1$ [see Eqs. (3.20) and (3.21)], in the large $N_{f}$ expansion. Any situation with nonzero Coulomb interaction and $z>1$ necessarily requires $\overline{w_{x}}$ and $\overline{\sigma_{e}}$ individually flowing to strong coupling. Note, however, that it is their dimensionless ratio $\overline{\sigma_{e}} / \overline{w_{x}}$ that appears in the action $S_{E}$ in the limit $\overline{w_{x}}, \overline{\sigma_{e}} \rightarrow \infty$. Consequently, we can parametrize this infinite Coulomb interaction limit with the marginal parameter $\alpha=\overline{\sigma_{e}} / \overline{w_{x}}$. We refer to $\alpha$ as the dissipation strength. $z>1$ is required for any fixed point with infinite Coulomb interaction to be IR attractive; treating $\alpha$ as a tuning parameter, we will view any infinite Coulomb interaction fixed point with $0<z<1$ as an IR unstable fixed point.

For $\overline{w_{x}} \rightarrow \infty, F_{w}$ in (3.19) reduces to

$$
F_{\infty}(\kappa, \alpha) \equiv \int_{-\infty}^{\infty} d y \frac{g_{1} \sqrt{1+y^{2}}\left(-1+2 y^{2}\right) \alpha+\left(1+y^{2}\right)|y|}{4 \pi^{2} N_{f}\left(1+y^{2}\right)^{\frac{5}{2}}\left[\sqrt{1+y^{2}}\left(g_{1}^{2}+\kappa^{2}\right) \alpha+g_{1}|y|\right]} .
$$

For any $\kappa$ and $\alpha \geqslant 0,0 \leqslant F_{\infty}(\kappa, \alpha) \leqslant \frac{8}{N_{f} \pi^{2}}$. In this limit, the dynamical critical exponent at infinite Coulomb coupling is

$$
z_{\infty}=1+\overline{g_{m}}+\overline{g_{0}}+2 \overline{g_{j}}-\phi_{1} \overline{g_{0}}-F_{\infty}(\kappa, \alpha),
$$

where we have explicitly indicated with $\phi_{1}$ how screening appears in $z_{\infty}$.

\section{1. $\mathcal{C}$ symmetry}

At infinite Coulomb coupling and in the presence of charge-conjugation symmetry, there exist nontrivial fixed points for any $\kappa$. These occur at small values of $\overline{g_{m}}$ and are found by solving $\beta_{\overline{g_{m}}}=0$ from (3.24) using Eq. (3.46):

$$
\begin{aligned}
{\overline{g_{m}}}^{*}(\alpha) & =F_{m}(\alpha, \kappa) \\
& \equiv \int_{-\infty}^{\infty} d y \frac{\sqrt{1+y^{2}}|y|+g_{1} \alpha\left(3+2 y^{2}\right)}{4 N_{f} \pi^{2}\left(1+y^{2}\right)^{2}\left[g_{1}|y|+\alpha \sqrt{1+y^{2}}\left(g_{1}^{2}+\kappa^{2}\right)\right]}-\frac{\alpha\left[g_{1} \sqrt{1+y^{2}}|y|+\left(1+y^{2}\right)\left(g_{1}^{2}-\kappa^{2}\right) \alpha\right]}{8 N_{f} \pi^{2}\left(1+y^{2}\right)^{\frac{3}{2}}\left[g_{1}|y|+\alpha \sqrt{1+y^{2}}\left(g_{1}^{2}+\kappa^{2}\right)\right]^{2}} .
\end{aligned}
$$

Figure 7 shows the behavior of the renormalization-group flows for $\kappa=0$ and $1 / 4 \pi$.

Note that the beta functions for $\overline{w_{x}} \rightarrow \infty$ are different from the case of finite $\overline{w_{x}}$, even when $\alpha=0$ : nontrivial fixed points exist for any value of $\kappa$. The correlation length exponent $v^{-1}=1$ because the fixed points are solved from $\beta_{\overline{g_{m}}}=0$ [see
Eq. (3.29)]. The dynamical critical exponent $z_{\infty}^{*}$ is found using (3.46):

$$
z_{\infty}^{*}(\alpha)={\overline{g_{m}}}^{*}(\alpha)-F_{\infty}(\kappa, \alpha) .
$$

To guarantee the irrelevancy of the diffusion constant $D_{e}$ of the 2 DEG bath, $z_{\infty}^{*}<2$ is required. Fortunately, $z_{\infty}^{*}$ does 


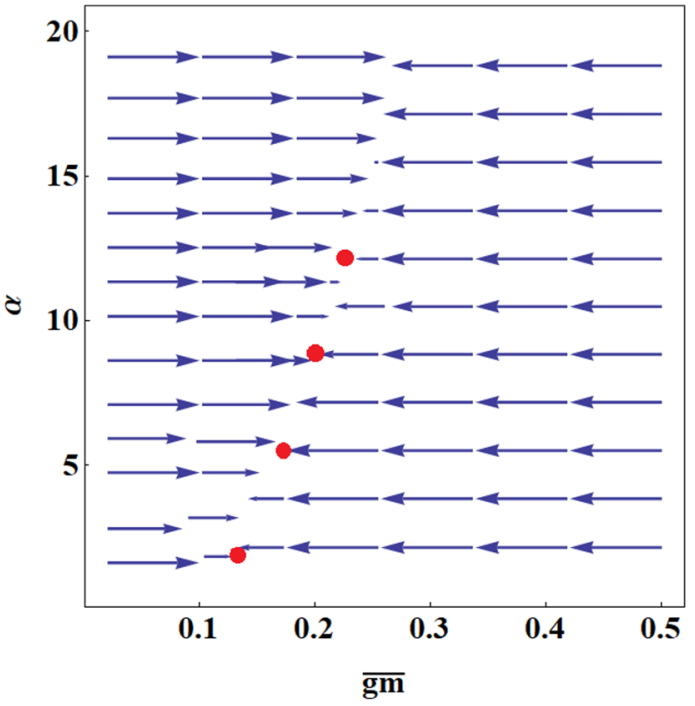

(a)

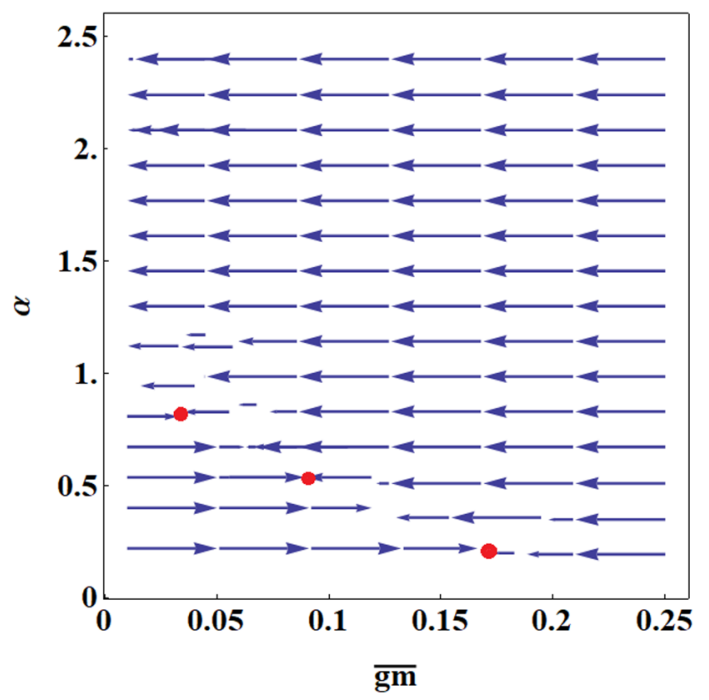

(b)

FIG. 7. RG flow of $\alpha$ vs $\overline{g_{m}}$. The dissipation parameter $\alpha$ is exactly marginal, so it is a free parameter that can be tuned. In the above figure, we choose $N_{f}=4$. (a) $\kappa=0$. (b) $\kappa=\frac{1}{4 \pi}$.

not exceed 2 for the values of $\alpha$ we have considered-see Fig. 8 .

\section{2. $\mathcal{C} \mathcal{T}$ symmetry}

As with finite Coulomb coupling, we focus on $\kappa=0$ in this and the next subsection because the Chern-Simons term is odd under $\mathcal{C} \mathcal{T}$ and $\mathcal{T}$.

In the $\overline{w_{x}} \rightarrow \infty$ limit, only $\beta_{\overline{g_{0}}}=0$ is nontrivial:

$$
2{\overline{g_{0}}}^{2}\left(-1+\phi_{1}\right)+2 \overline{g_{0}} F_{\infty}(\kappa=0, \alpha)=0 .
$$

Including disorder screening $\left(\phi_{1}=1\right), \overline{g_{0}}$ is marginally irrelevant. If disorder screening is ignored, an unstable fixed point lies at $\overline{g_{0}} *=F_{\infty}(\kappa=0, \alpha)$. Perturbation by $\overline{g_{0}}$ about this fixed point results either in flow (along the $\overline{g_{0}}$ direction) towards strong coupling or towards the infinite Coulomb interaction clean fixed point with critical exponents:

$$
\begin{gathered}
z_{\infty}(\alpha)=1-F_{\infty}(\kappa=0, \alpha)<1, \\
v^{-1}=1-F_{m}(\alpha, \kappa=0) .
\end{gathered}
$$

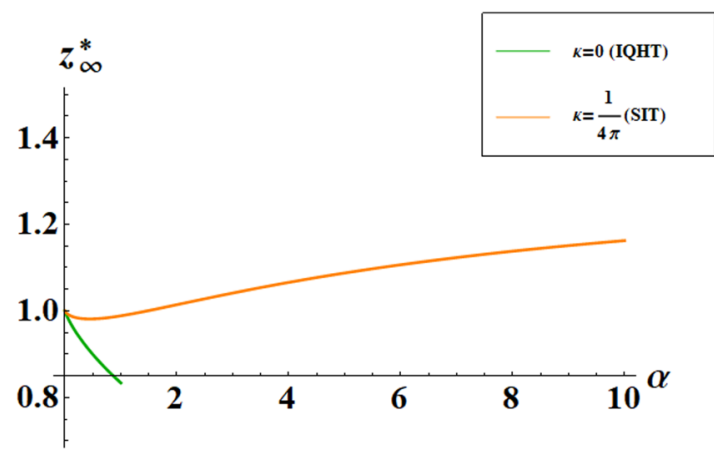

FIG. 8. Dynamical critical exponent $z_{\infty}^{*}$ vs effective dissipation strength $\alpha$ evaluated on the fixed points when $N_{f}=4$. The green line corresponds to $z_{\infty}^{*}<1$, which is an unstable $\overline{w_{x}} \rightarrow \infty$ fixed point. When $\kappa=1 / 4 \pi, 1<z_{\infty}^{*}<2$ for $\alpha \geqslant 1.47$.
Since $z_{\infty}<1$, these infinite Coulomb coupling fixed points are IR unstable. This renormalization-group flow is shown in Fig. 9.

\section{3. $\mathcal{T}$ symmetry}

When time-reversal symmetry is preserved and $\kappa=0$, the only nontrivial beta function is $\beta_{\overline{\Delta_{0}}}$. In the limit of strong Coulomb coupling, the disorder screening terms vanish. Solving $\beta_{\overline{\Delta_{0}}}=0$ gives the condition

$$
{\overline{g_{j}}}^{*}=\frac{1}{2} F_{\infty}(\alpha, \kappa=0)
$$

on the marginal couplings $\overline{g_{j}}$ and $\alpha$. The resulting fixed point is IR unstable along the $\overline{\Delta_{0}}$ direction and, depending on the values of $\overline{g_{j}}$ and $\alpha$, flows either to strong coupling or to

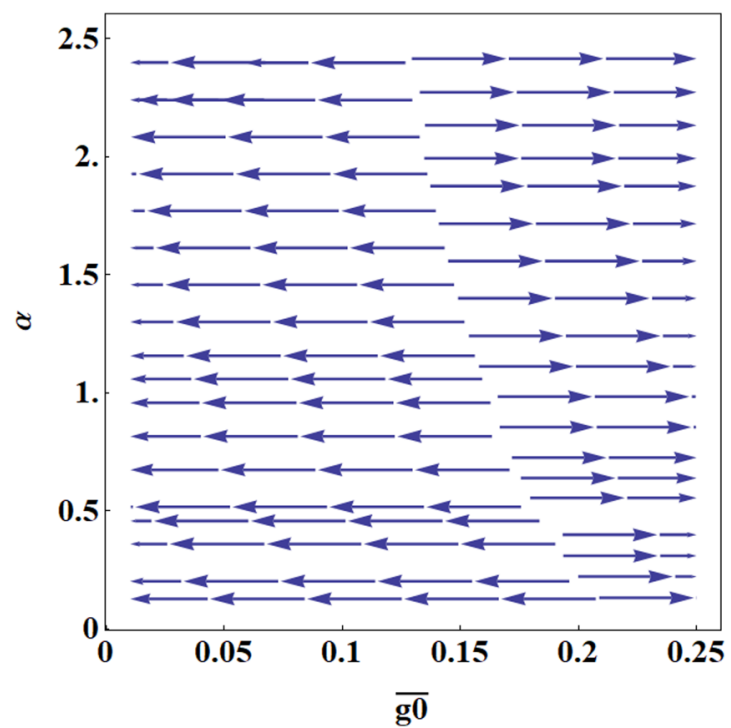

FIG. 9. The RG flow of $\overline{g_{0}}$ with respect to $\alpha$, where $\kappa=0$ is used. 


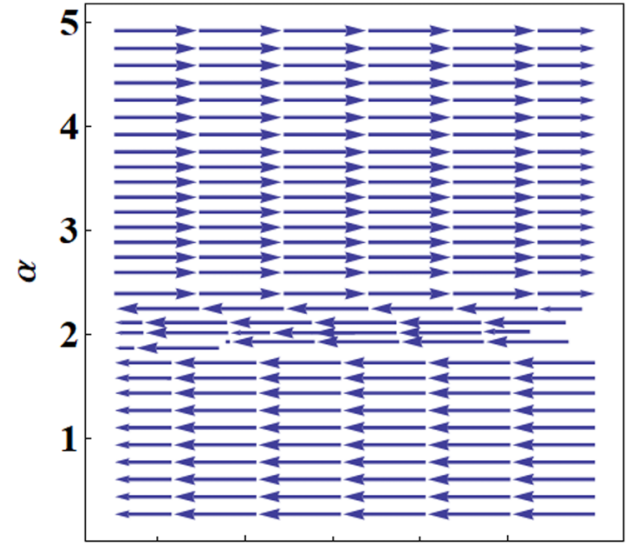

$\begin{array}{lllll}0.1 & 0.2 & 0.4 & 0.6 & 0.8\end{array}$

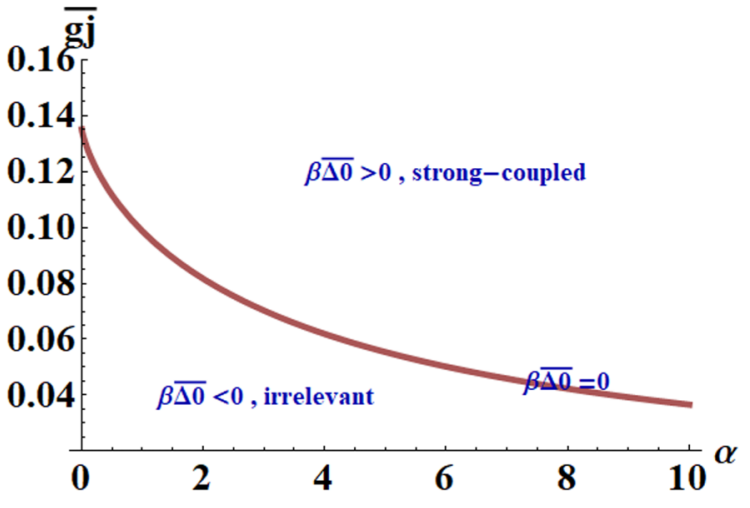

(b)

(a)

FIG. 10. The case of $\overline{w_{x}} \rightarrow \infty$ with $\mathcal{T}$ symmetry. (a) RG flow of $\overline{\Delta_{0}}$ vs $\alpha$ for setting $\overline{g_{j}}=0.08$. (b) The behavior of $\beta_{\overline{\Delta_{0}}}$ when adjusting $\overline{g_{j}}$ and $\alpha$.

zero when perturbed about this fixed point. This is shown in Fig. 10. Using Eq. (3.46), we see that $z_{\infty}=1$ at the fixed point defined by $\beta_{\overline{\Delta_{0}}}=0$. The correlation length exponent is given by $v^{*-1}=1+2{\overline{g_{j}}}^{*}-F_{m}(\alpha, \kappa=0)-\frac{{\overline{g_{j}}}^{*}}{g_{1}}=$ $1-7 F_{\infty}(\alpha, \kappa=0)-F_{m}(\alpha, \kappa=0)$, where the second equality is obtained after evaluating on the fixed point defined by Eq. (3.52).

\section{DISCUSSION}

In this paper, we studied the influence of quenched disorder and a dissipative Coulomb interaction on two different quantum phase transitions: an integer quantum Hall transition and a superconductor-insulator transition. We considered both transitions using effective theories that consist of a Dirac fermion coupled to a $U$ (1) Chern-Simons gauge field at level $(\theta-1 / 2): \theta=1 / 2$ corresponds to the IQHT, while $\theta=1$ corresponds to the SIT. We studied the critical properties of these theories using a renormalization-group analysis in which the number of fermion flavors $N_{f} \rightarrow \infty$. We found both theories to be stable to the addition of a Coulomb interaction. The IQHT was stable to $\mathcal{C}$ preserving disorder and exhibited a line of diffusive fixed points with $\mathcal{C}$ disorder. ( $\mathcal{C}$ is chargeconjugation symmetry and $\mathcal{T}$ is time-reversal symmetry.) The SIT exhibited a line of fixed points parametrized by the strength of dissipation when $\mathcal{C}$ is preserved. Other cases resulted in runaway flow.

Without disorder, the free Dirac fermion in (1.1) has a correlation length exponent $v_{\text {Dirac }}^{-1}=1$, while the $3 \mathrm{D} X Y$ model in (1.3) has a correlation length exponent $v_{\mathrm{XY}}^{-1} \approx 3 / 2$. In the large $N_{f}$ expansion, we find $v^{-1}(\theta=1 / 2) \approx 1-4.3 / N_{f}$ and $v^{-1}(\theta=1) \approx 1+1.4 / N_{f}$, in agreement with $[25,67,76,77]$. Evidently the leading-order term in the large $N_{f}$ expansion provides a poor approximation to the critical exponents of the clean fixed points [25]. Comparing our results $\left(v^{-1}=1, z=\right.$ $1+1.4 / N_{f}$ ) for the correlation length and dynamical critical exponents with recent numerical $(v \approx 1.2, z \approx 1.5)[39-42]$ and analytic $\left(v=1, z \approx 1+0.5 / N_{f}\right)$ [38] studies (without a Coulomb interaction) suggests this may also be the case for the dirty 3D XY model. Higher-order $\mathcal{O}\left(1 / N_{f}^{2}\right)$ terms may improve the comparison. Interestingly, the free Dirac fermion and 3D XY model admit duals involving a Dirac fermion coupled to a non-Abelian $U(N)$ Chern-Simons gauge field for any integer $N>1$ [78,79]. Such formulations suggest alternative approximation schemes. For instance, without disorder, these theories have a correlation length exponent equal to unity at two-loop order in the planar limit [72]. Importantly, the $U(N)$ generalization does not introduce additional global symmetry, in contrast to the large flavor approximation considered in this paper. Might the planar limit furnish better approximations to such theories, compared with the large $N_{f}$ expansion?

There are a variety of other observables and generalizations to consider. For example, scaling dimensions of the lowest dimension monopole operators in the Chern-Simons theories we studied should correspond to the $\eta$ exponents of the Dirac and XY models. The operator mapping constructed in [80] could prove useful for determining this. In $(2+1)$ dimensions, the dc $T \rightarrow 0$ conductivity tensor can be universal [43,81]; it would be interesting to calculate and compare across the duality [82]. Perhaps considering the behavior of a finite density of Dirac composite fermions is most pressing, given that the electronic systems inspiring this paper have a finite density of states.

One of the motivations of the current paper was to better understand the emergent symmetries that are found at IQHTs and SITs via electrical transport experiments. For concreteness, consider the magnetic field-tuned SIT at which a "selfduality" with dc $\sigma_{x x} \approx(2 e)^{2} / h$ and $\sigma_{x y} \approx 0$ is found at low temperatures [83]. ("Self-duality" requires that the electrical conductivity tensor of the 3D XY model satisfy $\sigma_{x x}^{2}+\sigma_{x y}^{2}=$ $\left(e_{*}^{2} / h\right)^{2}$, where $e_{*}$ is the electromagnetic charge of the bosons [84].) It was argued in [27] that PV symmetry (see Sec. II B) of the "fermionic dual" to the XY model in (2.1) results in selfdual transport. How this symmetry might be realized quantum mechanically is unclear [55]. This question is related to the 
emergent time-reversal symmetry of this "fermionic dual" at zero Dirac composite fermion density. Perhaps unsurprisingly, the leading-order large $N_{f}$ beta functions that we studied do not appear to respect the emergent time-reversal symmetry; at least, we have not found nontrivial solutions with an emergent time-reversal invariance at $\kappa=1 / 4 \pi$. It would be interesting to further understand this apparent shortcoming.

\section{ACKNOWLEDGMENTS}

We thank Hart Goldman, Sri Raghu, and Alex Thomson for useful conversations and correspondence. This material is based upon work supported by the US Department of Energy, Office of Science, Office of Basic Energy Sciences under Grant No. DE-SC0020007. This research was supported in part by the National Science Foundation under Grant No. NSF PHY-1748958.

\section{APPENDIX A: CALCULATION OVERVIEW}

In this Appendix we derive the residues $b_{\lambda_{a}}\left[\vec{\lambda}^{R}(\mu, \epsilon)\right]$ in Eq. (3.7),

$$
\lambda_{a}^{B} \mu^{-\Delta_{a}(\epsilon)}=\lambda_{a}^{R}(\mu, \epsilon)+\frac{b_{\lambda_{a}}\left[\vec{\lambda}^{R}(\mu, \epsilon)\right]}{\epsilon},
$$

that determine the beta functions at $\epsilon=0$ via Eq. (3.12):

$$
\beta_{\lambda_{a}}\left(\vec{\lambda}^{R}\right) \equiv-\mu \frac{\partial \lambda_{a}^{R}}{\partial \mu}=\bar{\Delta}_{\lambda_{a}} \lambda_{a}^{R}+\rho_{\lambda_{a}} b_{\lambda_{a}}\left(\vec{\lambda}^{R}\right)-\sum_{c} \rho_{\lambda_{c}} \lambda_{c}^{R} \frac{\partial b_{\lambda_{a}}\left(\vec{\lambda}^{R}\right)}{\partial \lambda_{c}^{R}} .
$$

After establishing notation, we will list the main results used in the main text. Later sections provide algebraic details.

\section{Setup}

Identify $S_{E}$ in Eqs. (3.2)-(3.4) with the bare action $S_{B}$ by endowing all fields and couplings with bare (B) subscripts/superscripts. To simplify notation, we will leave replica and flavor indices implicit. Define renormalized $(R)$ fields and couplings,

$$
\begin{aligned}
& \psi_{B}=Z_{f}^{1 / 2} \psi_{R}, \quad a_{B}^{0}=Z_{a, 0}^{1 / 2} a_{R}^{0}, \quad a_{B}^{j}=Z_{a, j}^{1 / 2} a_{R}^{j}, \\
& \lambda_{c}^{B}=Z_{c}^{-1 / 2} \lambda_{c}^{R}
\end{aligned}
$$

where the vector of couplings (either $B$ or $R$ )

$$
\vec{\lambda}=\left(\frac{g^{2}}{N_{f}}, v, m, \kappa, w_{x}, \sigma_{e}, D_{e}, g_{m}, g_{0}, g_{j}, \Delta_{0}, \Delta_{j}\right)^{T} .
$$

Separate $S_{B}$ into physical and counterterm actions:

$$
S_{B}=S_{\text {phys }}^{(1)}+S_{\text {phys }}^{(2)}+S_{\text {phys }}^{(3)}+S_{\mathrm{CT}}^{(1)}+S_{\mathrm{CT}}^{(2)}+S_{\mathrm{CT}}^{(3)}
$$

with

$$
\begin{gathered}
S_{\mathrm{phys}}^{(1)}=\int d \tau d^{D} x\left\{\bar{\psi}_{R}\left[\gamma_{\tau}\left(\partial^{\tau}+i \frac{g_{R} \mu^{\epsilon / 2}}{\sqrt{N_{f}}} a_{R}^{\tau}\right)+v_{R} \mu^{z-1} \gamma_{j}\left(\partial^{j}+i \frac{g_{R} \mu^{\epsilon / 2}}{\sqrt{N_{f}}} a_{R}^{j}\right)\right] \psi_{R}+\mu^{z} m_{R} \bar{\psi}_{R} \psi_{R}+\frac{i \kappa_{R}}{2} a_{R} d a_{R}\right\}, \\
S_{\mathrm{phys}}^{(2)}=\int d \omega d^{D} k \frac{w_{R} \mu^{z-1}}{2} a_{R}^{T}(-\omega,-k) \frac{k^{2}}{|k|+f_{R}(\omega, k)} a_{R}^{T}(\omega, k), \\
S_{\mathrm{phys}}^{(3)}=-\frac{1}{2} \int d \tau d \tau^{\prime} d^{D} x\left[\left(g_{m}\right)_{R} \mu^{2 z-D}\left(\bar{\psi}_{R} \psi_{R}\right)\left(\bar{\psi}_{R} \psi_{R}\right)+\left(g_{0}\right)_{R} \mu^{2 z-D}\left(\bar{\psi}_{R} \gamma^{0} \psi_{R}\right)\left(\bar{\psi}_{R} \gamma^{0} \psi_{R}\right)\right. \\
\left.+\left(g_{j}\right)_{R} \mu^{2 z-D}\left(\bar{\psi}_{R} \gamma^{j} \psi_{R}\right)\left(\bar{\psi}_{R} \gamma^{j} \psi_{R}\right)+\left(\Delta_{0}\right)_{R} \mu^{2 z-2} b_{R} b_{R}+\left(\Delta_{j}\right)_{R} \mathbf{e}_{R} \cdot \mathbf{e}_{R}\right], \\
S_{\mathrm{CT}}^{(1)}=\int d \tau d^{D} x\left\{\bar{\psi}_{R}\left[\gamma_{\tau}\left(\partial^{\tau}+i \frac{g_{R} \mu^{\epsilon / 2}}{\sqrt{N_{f}}} a_{R}^{\tau}\right) \delta_{1}+v_{R} \mu^{z-1} \gamma_{j}\left(\partial^{j}+i \frac{g_{R} \mu^{\epsilon / 2}}{\sqrt{N_{f}}} a_{R}^{j}\right) \delta_{2}\right] \psi_{R}+\mu^{z} m_{R} \bar{\psi}_{R} \psi_{R} \delta_{m}+\frac{i \kappa_{R}}{2} a_{R} d a_{R} \delta_{\kappa}\right\}, \\
S_{\mathrm{CT}}^{(2)}=\int d \omega d^{D} k \frac{w_{R} \mu^{z-1} \delta_{w}}{2} a_{R}^{T}(-\omega,-k) \frac{k^{2}}{|k|+f_{R}(\omega, k)} a_{R}^{T}(\omega, k), \\
S_{\mathrm{CT}}^{(3)}=-\frac{1}{2} \int d \tau d \tau^{\prime} d^{D} x\left[\left(g_{m}\right)_{R} \mu^{2 z-D} \delta_{g_{m}}\left(\bar{\psi}_{R} \psi_{R}\right)\left(\bar{\psi}_{R} \psi_{R}\right)+\left(g_{0}\right)_{R} \mu^{2 z-D} \delta_{g_{0}}\left(\bar{\psi}_{R} \gamma^{0} \psi_{R}\right)\left(\bar{\psi}_{R} \gamma^{0} \psi_{R}\right)\right. \\
\left.+\left(g_{j}\right)_{R} \mu^{2 z-D} \delta_{g_{j}}\left(\bar{\psi}_{R} \gamma^{j} \psi_{R}\right)\left(\bar{\psi}_{R} \gamma^{j} \psi_{R}\right)+\left(\Delta_{0}\right)_{R} \mu^{2 z-2} \delta_{\Delta_{0}} b_{R} b_{R}+\left(\Delta_{j}\right)_{R} \delta_{\Delta_{j}} \mathbf{e}_{R} \cdot \mathbf{e}_{R}\right], \\
f_{R}=\frac{e_{*}^{2}\left(\sigma_{e}\right)_{R} \mu^{z-1}}{|\omega|+\left(D_{e}\right)_{R} \mu^{z-2} k^{2}},
\end{gathered}
$$


and the renormalization-group scale $\mu$ enters in accord with the engineering dimensions listed in Table II. The counterterms $\delta_{X}$ have poles in $\epsilon$ with coefficients determined by the requirement that correlation functions of physical fields have no divergences as $\epsilon \rightarrow 0$. We focus exclusively on the terms in $\delta_{X}$ proportional to $1 / \epsilon$. Using Eq. (A3) to impose Eq. (A5), we relate the bare and renormalized couplings:

$$
\begin{gathered}
v^{B} \mu^{1-z}=v^{R}\left(1+\delta_{2}-\delta_{1}\right), \\
m^{B} \mu^{-z}=m^{R}\left(1+\delta_{m}-\delta_{1}\right), \\
\kappa^{B}=\kappa^{R}\left(1+\delta_{\kappa}\right), \\
w_{x}^{B} \mu^{1-z}=w_{x}^{R}\left(1+\delta_{w}\right), \\
g_{X}^{B} \mu^{D-2 z}=g_{X}^{R}\left(1-2 \delta_{1}+\delta_{g_{X}}\right), \quad g_{X} \in\left\{g_{m}, g_{0}, g_{j}\right\}, \\
\Delta_{0}^{B} \mu^{2-2 z}=\Delta_{0}^{R}\left(1+\delta_{\Delta_{0}}\right), \\
\Delta_{j}^{B}=\Delta_{j}^{R}\left(1+\delta_{\Delta_{j}}\right), \\
\sigma_{e}^{B} \mu^{1-z}=\sigma_{e}^{R}\left(1+\delta_{\sigma_{e}}\right), \\
D_{e}^{B}=D_{e}^{R}\left(1+\delta_{D}\right) .
\end{gathered}
$$

Thus, we can read off the residues:

$$
\begin{gathered}
b_{v}=v^{R}\left(\delta_{2}-\delta_{1}\right) \epsilon, \\
b_{m}=m^{R}\left(\delta_{m}-\delta_{1}\right) \epsilon, \\
b_{\kappa}=\kappa^{R} \delta_{\kappa} \epsilon, \\
b_{w}=w_{x}^{R} \delta_{w} \epsilon, \\
b_{g_{X}}=-g_{X}^{R}\left(2 \delta_{1}-\delta_{g_{X}}\right) \epsilon, \quad g_{X} \in\left\{g_{m}, g_{0}, g_{j}\right\}, \\
b_{\Delta_{X}}=\Delta_{X}^{R} \delta_{\Delta_{X}} \epsilon, \quad \Delta_{X} \in\left\{\Delta_{0}, \Delta_{j}\right\}, \\
b_{\sigma_{e}}=\sigma_{e}^{R} \delta_{\sigma} \epsilon, \\
b_{D_{e}}=D_{e}^{R} \delta_{D} \epsilon .
\end{gathered}
$$

\section{APPENDIX B: COUNTERTERMS}

As discussed in the main text, we choose the dynamical critical exponent $z$ in such a way that the fermion velocity $v$ does not run, i.e., the velocity beta function is zero. In the expressions below, it is convenient to redefine couplings to absorb the velocity dependence as follows:

$$
\begin{aligned}
\overline{g_{m}} & =\frac{g_{m}}{2 \pi v^{2}}, \quad \overline{g_{0}}=\frac{g_{0}}{2 \pi v^{2}}, \quad \overline{g_{j}}=\frac{g_{j}}{2 \pi v^{2}}, \quad \overline{\Delta_{0}}=\Delta_{0}, \\
\overline{\Delta_{j}} & =\Delta_{j} v^{2}, \quad \overline{w_{x}}=\frac{w_{x}}{v}, \quad \overline{\sigma_{e}}=\frac{\sigma_{e}}{v}, \quad \int_{-\infty}^{\infty} d z F(z) \\
& =\int_{-\infty}^{\infty} v d y F\left(y=\frac{z}{v}\right),
\end{aligned}
$$

where the function $F(z)$ is introduced below. We also define $g_{1}=g^{2} / 16$ and $\bar{m}=m / v$.

Let us make a few remarks about the expressions below.

(1) We use $\phi_{1}$ to parametrize the screening of the disorder described in Appendix C. $\phi_{1}=0$ means the screening is ignored; $\phi_{1}=1$ means the screening is included.

(2) Terms proportional to $\xi$ are divergent. However, this is an unphysical divergence due to our gauge choice: This divergence does not appear in physical quantities such as critical exponents.

(3) The Ward identity guarantees that the gauge field corrections to $\delta_{1}$ and $\delta g_{0}$ cancel; the ones in $\delta_{2}$ and $\delta_{g_{j}}$ likewise cancel. In the absence of the Coulomb interaction, the equality of the gauge corrections in $\delta_{1}$ and $\delta_{2}$ is a coincidence, which makes $\beta_{g_{0}}$ and $\beta_{g_{j}}$ independent of the gauge corrections. When the Coulomb interaction is included, $\beta_{g_{0}}$ receives corrections from the gauge field, while $\beta_{g_{j}}$ does not. 

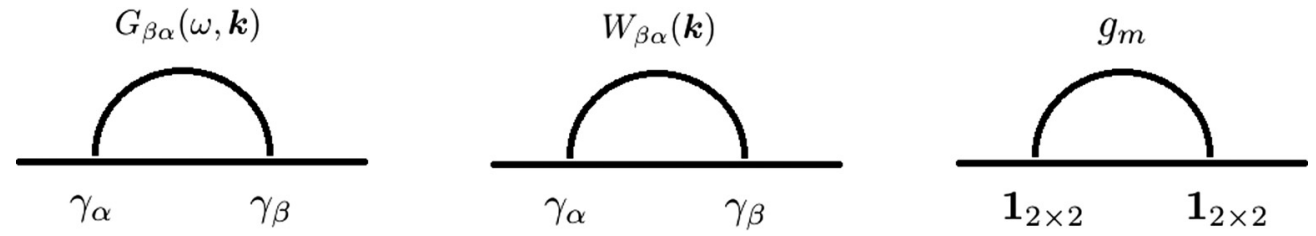

FIG. 11. Diagrams contributing to $\delta_{1}$ and $\delta_{2}$.

1. $\delta_{\kappa}, \delta_{w}, \delta_{\sigma}$, and $\delta_{D}$ counterterms

Quantization of the Chern-Simons level and finiteness of the gauge field self-energy in three dimensions implies

$$
\delta_{\kappa}=\delta_{w}=\delta_{\sigma}=\delta_{D}=0 .
$$

Consequently, renormalizations of $\kappa, w_{x}, \sigma_{e}$, and $D_{e}$ are controlled by their engineering dimensions.

\section{2. $\delta_{1}$ counterterm}

The diagrams that contribute to $\delta_{1}$ are given by taking the temporal component of Fig. 11:

$$
\begin{aligned}
\delta_{1} \epsilon= & -\left(\overline{g_{m}}+\overline{g_{0}}+2 \overline{g_{j}}\right)+\phi_{1}\left[\frac{g_{1}^{2} \overline{w_{x}}\left(g_{1} \overline{g_{j}}-g_{1} \overline{g_{0}}-\overline{g_{0}} \overline{w_{x}}\right)}{\left(g_{1}^{2}+g_{1} \overline{w_{x}}+\kappa^{2}\right)^{2}}+\frac{g_{1}\left(g_{1} \overline{g_{j}}+g_{1} \overline{g_{0}}+2 \overline{g_{0}} \overline{w_{x}}\right)}{g_{1}^{2}+g_{1} \overline{w_{x}}+\kappa^{2}}\right] \\
& +\int_{-\infty}^{\infty} \frac{1}{4 \pi^{2} N_{f}} \frac{\left(1-y^{2}\right)\left(g_{1} \overline{\sigma_{e}} y^{2}+\overline{w_{x}} \sqrt{1+y^{2}}|y|\right)+g_{1}|y| y^{2}\left(1-y^{2} \xi\right)}{\left(1+y^{2}\right)^{2}\left[\sqrt{1+y^{2}}\left(g_{1}^{2}+\kappa^{2}\right)\left(\overline{\sigma_{e}}+|y|\right)+g_{1} \overline{w_{x}}|y|\right]} .
\end{aligned}
$$

\section{3. $\delta_{2}$ counterterm}

The diagrams that contribute to $\delta_{2}$ are given by taking the spatial component of Fig. 11:

$$
\begin{aligned}
\delta_{2} \epsilon= & -\phi_{1} \frac{g_{1}^{2}\left[g_{1}^{2} \overline{g_{j}}+2 g_{1} \overline{g_{j}} \overline{w_{x}}-\left(\overline{g_{0}}-2 \overline{g_{j}}\right) \kappa^{2}\right]}{\left(g_{1}^{2}+g_{1} \overline{w_{x}}+\kappa^{2}\right)^{2}} \\
& +\int_{-\infty}^{\infty} d y \frac{1}{4 \pi^{2} N_{f}} \frac{g_{1}\left(1-y^{2}-y^{4}\right) \overline{\sigma_{e}}-\left(\overline{w_{x}} y^{2} \sqrt{1+y^{2}}\right)|y|+g_{1}\left(1-y^{2}-y^{4} \xi\right)|y|}{\left(1+y^{2}\right)^{2}\left[\sqrt{1+y^{2}}\left(g_{1}^{2}+\kappa^{2}\right)\left(\overline{\sigma_{e}}+|y|\right)+g_{1} \overline{w_{x}}|y|\right]} .
\end{aligned}
$$

\section{4. $\delta_{g_{m}}$ counterterm}

$\delta_{g_{m}}$ is extracted from the diagrams in Figs. 12 and 13:

$$
\begin{aligned}
& \delta_{g_{m}} \epsilon=\left[\frac{2\left(\overline{g_{0}}+\overline{g_{m}}\right)\left(\overline{g_{m}}-2 \overline{g_{j}}\right)}{\overline{g_{m}}}+2 \frac{g_{1}\left(\overline{g_{j}}-\overline{g_{0}}\right)+\overline{g_{j}} \overline{w_{x}}}{g_{1}^{2}+g_{1} \overline{w_{x}}+\kappa^{2}}\right] \\
& +\phi_{1}\left[4 g_{1} \frac{g_{1} \kappa^{2}\left(-{\overline{g_{0}}}^{2}-{\overline{g_{j}}}^{2}\right)+\overline{g_{j}} \overline{g_{0}}\left[2 g_{1}^{3}+4 g_{1}^{2} \overline{w_{x}}+2 \overline{w_{x}} \kappa^{2}+g_{1}\left({\overline{w_{x}}}^{2}+4 \kappa^{2}\right)\right]}{\overline{g_{m}}\left[g_{1}^{2}+g_{1} \overline{w_{x}}+\kappa^{2}\right]^{2}}\right] \\
& +\phi_{1}\left[\frac{g_{1}^{5}\left(\overline{g_{0}}-\overline{g_{j}}\right)+g_{1}^{4} \overline{w_{x}}\left(2 \overline{g_{0}}-3 \overline{g_{j}}\right)+g_{1}^{3}\left(\overline{g_{0}}{\overline{w_{x}}}^{2}-2 \overline{g_{j}}{\overline{w_{x}}}^{2}+3 \overline{g_{0}} \kappa^{2}-3 \overline{g_{j}} \kappa^{2}\right)}{\left[g_{1}^{2}+g_{1} \overline{w_{x}}+\kappa^{2}\right]^{3}}+\frac{g_{1}^{2} \kappa^{2}\left(3 \overline{g_{0}} \overline{w_{x}}\right)+2 g_{1} \kappa^{4}\left(\overline{g_{j}}-\overline{g_{0}}\right)}{\left[g_{1}^{2}+g_{1} \overline{w_{x}}+\kappa^{2}\right]^{3}}\right] \\
& +\phi_{1}\left[\frac{2 g_{1}^{4}\left(\overline{g_{j}}-\overline{g_{0}}\right)+4 g_{1}^{3} \overline{w_{x}}\left(\overline{g_{j}}-\overline{g_{0}}\right)+g_{1}^{2}\left(-2 \overline{g_{0}}{\overline{w_{x}}}^{2}-6 \overline{g_{0}} \kappa^{2}+6 \overline{g_{j}} \kappa^{2}\right)-4 g_{1} \overline{g_{0}} \overline{w_{x}} \kappa^{2}}{\left[g_{1}^{2}+g_{1} \overline{w_{x}}+\kappa^{2}\right]^{2}}\right] \\
& +\phi_{1}^{2}\left[\frac{4 g_{1}^{2}{\overline{g_{j}}}^{2} \kappa^{2}\left[g_{1}^{2}\left(g_{1}^{2}+2 g_{1} \overline{w_{x}}-{\overline{w_{x}}}^{2}\right)+2 g_{1}\left(g_{1}-\overline{w_{x}}\right) \kappa^{2}-\kappa^{4}\right]}{\overline{g_{m}}\left[g_{1}^{2}+g_{1} \overline{w_{x}}+\kappa^{2}\right]^{4}}\right. \\
& +\frac{4 g_{1}^{2}{\overline{g_{0}}}^{2} \kappa^{2}\left[g_{1}^{2}\left(g_{1}^{2}+2 g_{1} \overline{w_{x}}+{\overline{w_{x}}}^{2}\right)+2 g_{1}\left(g_{1}+\overline{w_{x}}\right) \kappa^{2}-\kappa^{4}\right]}{\overline{g_{m}}\left[g_{1}^{2}+g_{1} \overline{w_{x}}+\kappa^{2}\right]^{4}}
\end{aligned}
$$



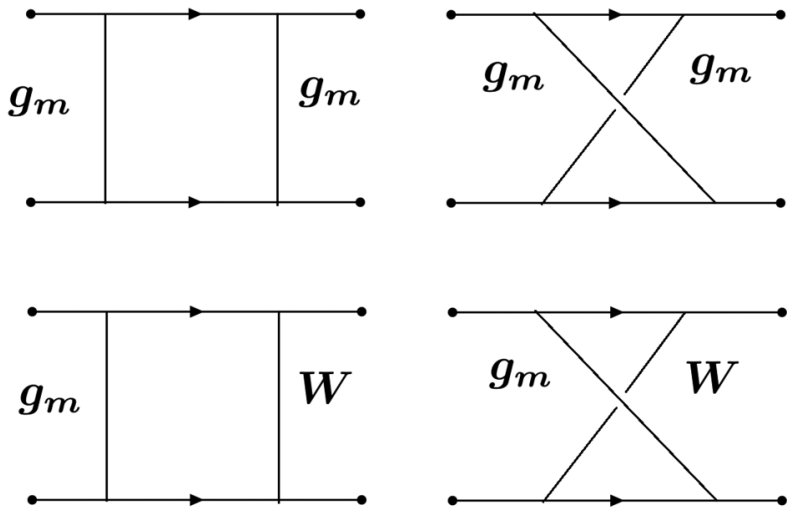
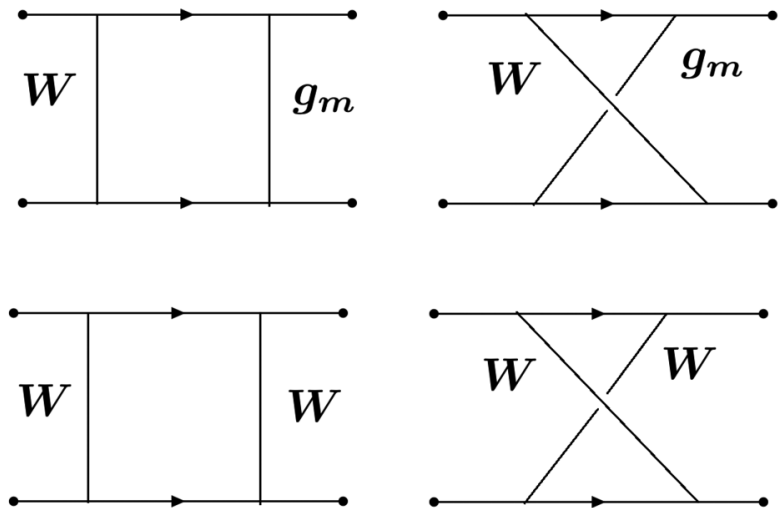

FIG. 12. Two particle-irreducible (2-PI) diagrams contributing to $\delta_{g_{m}}, \delta_{g_{0}}$, and $\delta_{g_{j}}$.

$$
\begin{aligned}
& \left.+\frac{4 \overline{g_{0}} \overline{g_{j}} g_{1}^{2}\left[-g_{1}^{3}\left(g_{1}+\overline{w_{x}}\right)^{2}\left(g_{1}+2 \overline{w_{x}}\right)-2 g_{1}^{2}\left(g_{1}+\overline{w_{x}}\right)\left(2 g_{1}+3 \overline{w_{x}}\right) \kappa^{2}-g_{1}\left(5 g_{1}+2 \overline{w_{x}}\right) \kappa^{4}+2 \kappa^{6}\right]}{\overline{g_{m}}\left[g_{1}^{2}+g_{1} \overline{w_{x}}+\kappa^{2}\right]^{4}}\right] \\
& -\int_{-\infty}^{\infty} d y \frac{g_{1}\left(y^{2}+2\right)\left(1+y^{2}\right) \overline{\sigma_{e}}+\left[\overline{w_{x}}\left(1+y^{2}\right) \sqrt{1+y^{2}}+g_{1}\left(2+3 y^{2}+y^{4} \xi\right)\right]|y|}{2 \pi^{2} N_{f}\left(1+y^{2}\right)^{2}\left[\sqrt{1+y^{2}}\left(g_{1}^{2}+\kappa^{2}\right)\left(\overline{\sigma_{e}}+|y|\right)+g_{1} \overline{w_{x}}|y|\right]} \\
& +\int_{-\infty}^{\infty} d y \frac{\left(\overline{\sigma_{e}}+|y|\right)\left[\left(1+y^{2}\right)\left(g_{1}^{2}-\kappa^{2}\right)\left(\overline{\sigma_{e}}+|y|\right)+\left(g_{1} \overline{w_{x}} \sqrt{1+y^{2}}\right)|y|\right]}{4 \pi^{2} N_{f}\left(1+y^{2}\right)^{\frac{3}{2}}\left[\sqrt{1+y^{2}}\left(g_{1}^{2}+\kappa^{2}\right)\left(\overline{\sigma_{e}}+|y|\right)+g_{1} \overline{w_{x}}|y|\right]^{2}} .
\end{aligned}
$$

\section{5. $\delta_{m}$ counterterm}

$\delta_{m}$ is extracted from the diagrams in Fig. 13:

$$
\begin{aligned}
m \epsilon \delta_{m}= & \frac{1}{2} g_{m} \epsilon\left(\delta_{g_{m}}-2 \text {-PI box }\right) \\
= & \overline{g_{0}}-2 \overline{g_{j}}+\overline{g_{m}}-\frac{g_{1}\left(\overline{g_{0}}-\overline{g_{j}}\right)-\overline{g_{j}} \overline{w_{x}}}{2\left(g_{1}^{2}+g_{1} \overline{w_{x}}+\kappa^{2}\right)}+\phi_{1}\left(-\frac{g_{1}\left(\overline{g_{0}}-\overline{g_{j}}\right)+3 g_{1}\left(\overline{g_{0}}-\overline{g_{j}}\right)+2 \overline{g_{0}} \overline{w_{x}}}{g_{1}^{2}+g_{1} \overline{w_{x}}+\kappa^{2}}\right. \\
& -g_{1}^{2} \frac{\overline{g_{j}}\left[4 g_{1}^{2}+4 \overline{w_{x}}+g_{1}\left(7+2 \overline{w_{x}}\right)\right]-\overline{g_{0}}\left[4 g_{1}^{2}+\overline{w_{x}}\left(7+2 \overline{w_{x}}+\kappa^{2}\right)^{2}\right.}{\left.g_{1}\left(7+6 \overline{w_{x}}\right)\right]} \\
& \left.-\frac{-2 g_{1}^{3}\left(g_{1}+\overline{w_{x}}\right)\left[-g_{1} \overline{g_{j}}+\overline{g_{0}}\left(g_{1}+\overline{w_{x}}\right)\right]}{\left(g_{1}^{2}+g_{1} \overline{w_{x}}+\kappa^{2}\right)^{3}}\right) \\
& -\int_{-\infty}^{\infty} d y \frac{g_{1}\left(y^{2}+2\right)\left(1+y^{2}\right) \overline{\sigma_{e}}+\left(\overline{w_{x}}\left(1+y^{2}\right) \sqrt{1+y^{2}}+g_{1}\left(2+3 y^{2}+y^{4} \xi\right)\right)|y|}{4 \pi^{2} N_{f}\left(1+y^{2}\right)^{2}\left[\sqrt{1+y^{2}}\left(g_{1}^{2}+\kappa^{2}\right)\left(\overline{\sigma_{e}}+|y|\right)+g_{1} \overline{w_{x}}|y|\right]} \\
& +\int_{-\infty}^{\infty} d y \frac{\left(\overline{\sigma_{e}}+|y|\right)\left[\left(1+y^{2}\right)\left(g_{1}^{2}-\kappa^{2}\right)\left(\overline{\sigma_{e}}+|y|\right)+\left(g_{1} \overline{w_{x}} \sqrt{1+y^{2}}\right)|y|\right]}{8 \pi^{2} N_{f}\left(1+y^{2}\right)^{\frac{3}{2}}\left[\sqrt{1+y^{2}}\left(g_{1}^{2}+\kappa^{2}\right)\left(\overline{\sigma_{e}}+|y|\right)+g_{1} \overline{w_{x}}|y|\right]^{2}},
\end{aligned}
$$

where [two particle-irreducible (2-PI) boxes] refers to the contributions to $\delta_{g_{m}}$ from the diagrams in Fig. 12.
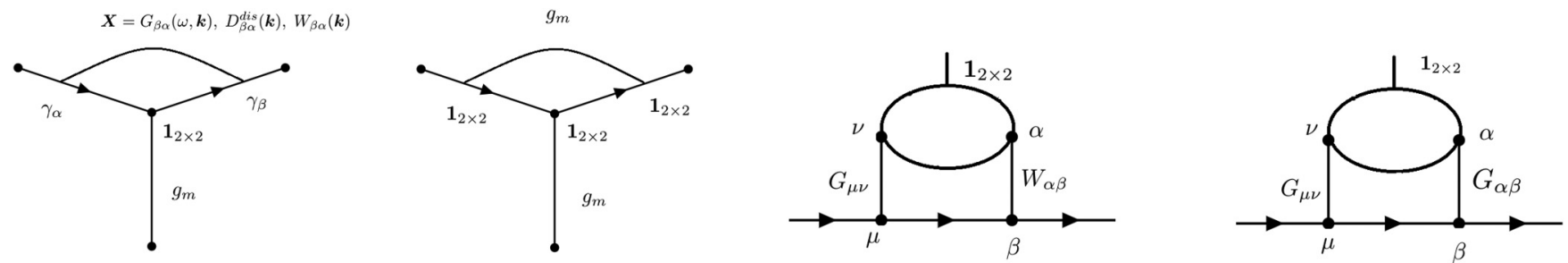

FIG. 13. The mass vertex contributions to $\delta_{g_{m}}$. 


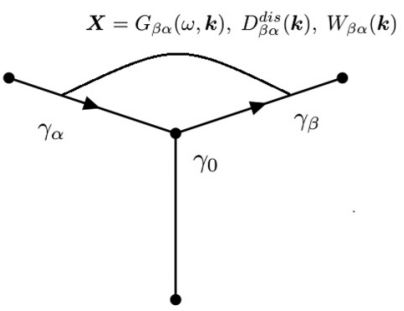

FIG. 14. $\gamma_{\mu=0}$ vertex component contributions to $\delta_{g_{0}}$.
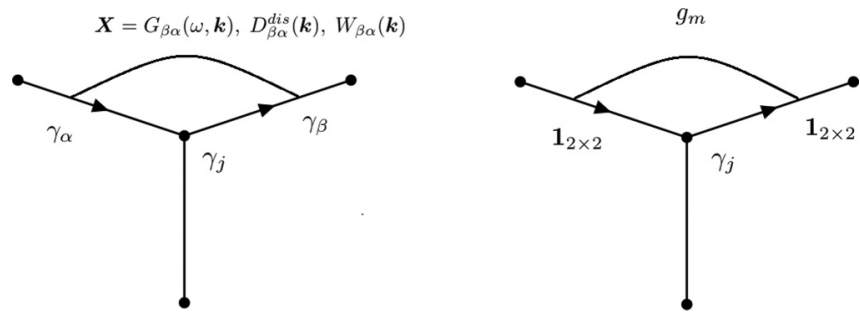

FIG. 15. $\gamma_{\mu=j}$ vertex component contributions to $\delta_{g_{j}}$.

6. $\delta_{g_{0}}$ counterterm

$\delta_{g_{0}}$ is extracted from the diagrams in Figs. 12 and 14:

$$
\begin{aligned}
\delta_{g_{0}} \epsilon= & \frac{-2\left(\overline{g_{0}}+2 \overline{g_{j}}\right)\left(\overline{g_{0}}+\overline{g_{m}}\right)}{\overline{g_{0}}}+\phi_{1}\left[\frac{4 g_{1}^{2} \overline{g_{j}} \overline{g_{m}}\left(g_{1}^{2}+2 g_{1} \overline{w_{x}}+2 \kappa^{2}\right)}{\overline{g_{0}}\left[g_{1}^{2}+g_{1} \overline{w_{x}}+\kappa^{2}\right]^{2}}+\frac{2 g_{1}^{2}\left[g_{1}^{2} \overline{g_{j}}+2 g_{1} \overline{g_{j}} \overline{w_{x}}+\left(\overline{g_{j}}-2 \overline{g_{m}}\right) \kappa^{2}\right]}{\left[g_{1}^{2}+g_{1} \overline{w_{x}}+\kappa^{2}\right]^{2}}\right. \\
& \left.+\frac{2 \overline{g_{0}} g_{1}\left(g_{1}^{3}+2 g_{1}^{2} \overline{w_{x}}+2 \kappa^{2} \overline{w_{x}}+g_{1} \overline{w_{x}}+g_{1} \kappa^{2}\right)}{\left[g_{1}^{2}+g_{1} \overline{w_{x}}+\kappa^{2}\right]^{2}}\right] \\
& +\int_{-\infty}^{\infty} d y \frac{g_{1} y^{2}\left(1-y^{2}\right) \overline{\sigma_{e}}+g_{1} y^{2}\left(1-y^{2} \xi\right)|y|+\left(1-y^{2}\right) \overline{w_{x}} \sqrt{1+y^{2}}|y|}{2 \pi^{2} N_{f}\left(1+y^{2}\right)^{2}\left[\sqrt{1+y^{2}}\left(g_{1}^{2}+\kappa^{2}\right)\left(\overline{\sigma_{e}}+|y|\right)+g_{1} \overline{w_{x}}|y|\right]} .
\end{aligned}
$$

\section{7. $\delta_{g_{j}}$ counterterm}

$\delta_{g_{j}}$ is extracted from the diagrams in Figs. 12 and 15:

$$
\begin{aligned}
\delta_{g_{j}} \epsilon= & \frac{-2 \overline{g_{0}} \overline{g_{m}}}{\overline{g_{j}}}+\phi_{1}\left[\frac{2 g_{1}^{2}\left(\overline{g_{0}}-\overline{g_{m}}\right) \kappa^{2}}{\left[g_{1}^{2}+g_{1} \overline{w_{x}}+\kappa^{2}\right]^{2}}\right]+\phi_{1}\left[\frac{2 g_{1} \overline{g_{0}} \overline{g_{m}}\left(g_{1}^{3}+2 g_{1}^{2} \overline{w_{x}}+2 \overline{w_{x}} \kappa^{2}+g_{1} \overline{w_{x}}+2 g_{1} \kappa^{2}\right)}{\left[g_{1}^{2}+g_{1} \overline{w_{x}}+\kappa^{2}\right]^{2}}\right] \\
& +\int_{-\infty}^{\infty} d y \frac{1}{2 \pi^{2} N_{f}} \frac{g_{1}\left(1-y^{2}-y^{4}\right) \overline{\sigma_{e}}-\left(\overline{w_{x}} y^{2} \sqrt{1+y^{2}}\right)|y|+g_{1}\left(1-y^{2}-y^{4} \xi\right)|y|}{\left(1+y^{2}\right)^{2}\left[\sqrt{1+y^{2}}\left(g_{1}^{2}+\kappa^{2}\right)\left(\overline{\sigma_{e}}+|y|\right)+g_{1} \overline{w_{x}}|y|\right]} .
\end{aligned}
$$

\section{8. $\delta_{\Delta_{0}}$ counterterm}

$\delta_{\Delta_{0}}$ is extracted from the diagram in Fig. 16:

$$
\begin{aligned}
\delta_{\Delta_{0}} \epsilon= & \frac{-\overline{g_{m}}\left(g_{1}^{2} \overline{\Delta_{j}}+2 g_{1} \overline{w_{x}} \overline{\Delta_{j}}+\overline{w_{x}} \overline{\Delta_{j}}+\overline{\Delta_{0}} \kappa^{2}\right)}{64 \overline{\Delta_{0}}\left(g_{1}^{2}+g_{1} \overline{w_{x}}+\kappa^{2}\right)^{2}} \\
& +\left[\frac{-\overline{g_{0}} \overline{g_{m}} N_{f} \pi v^{2}}{32 \overline{\Delta_{0}}}+\phi_{1} \frac{g_{1} \overline{g_{m}} N_{f} \pi v^{2}\left[-g_{1} \overline{g_{j}} \kappa^{2}+\overline{g_{0}}\left(g_{1}+\overline{w_{x}}\right)\left(g_{1}^{2}+g_{1} \overline{w_{x}}+2 \kappa^{2}\right)\right]}{32 \overline{\Delta_{0}}\left(g_{1}^{2}+g_{1} \overline{w_{x}}+\kappa^{2}\right)^{2}}\right] .
\end{aligned}
$$

\section{9. $\delta_{\Delta_{j}}$ counterterm}

$\delta_{\Delta_{j}}$ is extracted from the diagram in Fig. 16:

$$
\delta_{\Delta_{j}} \epsilon=\frac{-\overline{g_{m}}\left(g_{1}^{2} \overline{\Delta_{0}}+\overline{\Delta_{j}} \kappa^{2}\right)}{128 \overline{\Delta_{j}}\left(g_{1}^{2}+g_{1} \overline{w_{x}}+\kappa^{2}\right)^{2}}+\left[\frac{-\overline{g_{j}} \overline{g_{m}} N_{f} \pi v^{2}}{64 \overline{\Delta_{j}}}+\phi_{1} \frac{g_{1}^{2} \overline{g_{m}} N_{f} \pi v^{2}\left[g_{1}^{2} \overline{g_{j}}+2 g_{1} \overline{g_{j}} \overline{w_{x}}-\left(\overline{g_{0}}-2 \overline{g_{j}}\right) \kappa^{2}\right]}{64 \overline{\Delta_{j}}\left(g_{1}^{2}+g_{1} \overline{w_{x}}+\kappa^{2}\right)^{2}}\right] .
$$

\section{APPENDIX C: FEYNMAN RULES FOR DISORDER AND SCREENING}

\section{Feynman rules for disorder vertices}

From the action (A8), we can read the Feynman rules for the various types of disorder: (1) the four-fermion mass vertex,

$$
\frac{1}{2}(\bar{\psi} \psi)(\bar{\psi} \psi) \Rightarrow+g_{m} 2 \pi \delta(\omega=0) ;
$$




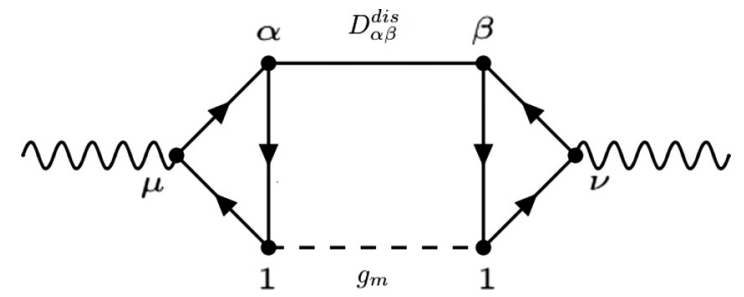

FIG. 16. Diagrams contributing to $\delta_{\Delta_{0}}$ and $\delta_{\Delta_{j}}$.

(2) the four-fermion density vertex,

$$
\frac{1}{2}\left(\bar{\psi} \gamma^{0} \psi\right)\left(\bar{\psi} \gamma^{0} \psi\right) \Rightarrow\left(g_{0}\right)\left(\gamma^{0}\right)\left(\gamma^{0}\right) 2 \pi \delta(\omega=0) ;
$$

(3) the four-fermion current vertex along the $k$ direction $k=x$ or $y$,

$$
\frac{1}{2}\left(\bar{\psi} i \gamma^{k} \psi\right)\left(\bar{\psi} i \gamma^{k} \psi\right) \Rightarrow\left(+g_{j}\right)\left(i \gamma^{k}\right)\left(i \gamma^{k}\right) 2 \pi \delta(\omega=0) ;
$$

(4) the $b(\tau) b\left(\tau^{\prime}\right)$ disordered two-point vertex rule,

$$
\frac{1}{2} b_{z} b_{z} \Rightarrow \Delta_{0}\left(\delta_{i j} \mathbf{k}^{2}-k_{i} k_{j}\right) 2 \pi \delta(\omega) a_{i}(\mathbf{k}) a_{j}(-\mathbf{k}) ;
$$

and (5) the $e_{j}(\tau) e_{j}\left(\tau^{\prime}\right)$ disordered two-point vertex,

$$
\frac{1}{2}\left(e_{x} e_{x}+e_{y} e_{y}\right) \Rightarrow\left(-\Delta_{j} c^{2}\right)\left(k_{x}^{2}+k_{y}^{2}\right) 2 \pi \delta(\omega) a_{0}(\mathbf{k}) a_{0}(-\mathbf{k}) .
$$

The factor of $\frac{1}{2}$ is canceled by a symmetry factor equal to 2 . The $2 \pi$ factor always cancels with the $1 / 2 \pi$ factor that accompanies any frequency integral $\int \frac{d \omega}{2 \pi}$.

\section{Gauge propagator}

\section{a. Vacuum polarization tensor}

$$
\begin{aligned}
\Pi_{\mu \nu}\left(k_{0}, \mathbf{k}\right) & =\left(\frac{-i g}{\sqrt{N_{f}}}\right)^{2}\left(\frac{v}{c}\right)^{2-\delta_{\mu 0}-\delta_{v 0}} \times N_{f} \times(-1) \int \frac{d^{2} p_{E}}{(2 \pi)^{3}} \operatorname{Tr}\left[\gamma_{\mu} S_{F}(k+p) \gamma_{\nu} S_{F}(p)\right] \\
& =\left(+g^{2}\right)(-i)^{2}\left(\frac{v}{c}\right)^{2-\delta_{\mu 0}-\delta_{\nu 0}} \frac{1}{v^{2}} \int \frac{d^{2} \bar{p}_{E} d p_{0}}{(2 \pi)^{3}} \operatorname{Tr}\left[\gamma_{\mu} \gamma_{\alpha} \gamma_{\nu} \gamma_{\beta}\right] \frac{(\bar{k}+\bar{p})_{\alpha}(\bar{p})_{\beta}}{\left[x(\bar{k}+\bar{p})^{2}+(1-x) \bar{p}^{2}\right]^{2}}, \\
\Pi_{\mu \nu}\left(k_{0}, \mathbf{k}\right) & =\frac{-1}{16} \frac{g^{2}}{v^{2}}\left(\frac{v}{c}\right)^{2-\delta_{\mu 0}-\delta_{\nu 0}} \frac{1}{|\bar{k}|}\left[\delta_{\mu \nu} \bar{k}^{2}-\bar{k}_{\mu} \bar{k}_{\nu}\right], \\
\bar{k} & =(\omega, v \mathbf{k}), \quad|\bar{k}|=\sqrt{\omega^{2}+v^{2} \mathbf{k}^{2}} .
\end{aligned}
$$

The minus sign comes from the fermion loop. The ratio $v / c$ can be set to $v$ in future equations.

\section{b. "1- $\mu$ " vacuum polarization vector}

$$
\begin{aligned}
\Pi_{\mu}\left(k_{0}, \mathbf{k}\right) & =\sqrt{g_{m}}\left(\frac{-i g}{\sqrt{N_{f}}}\right)\left(\frac{v}{c}\right)^{1-\delta_{\mu 0}} \times N_{f} \times(-1) \int \frac{d^{2} p_{E}}{(2 \pi)^{3}} \operatorname{Tr}\left[\gamma_{\mu} S_{F}(k+p) \mathbf{1} S_{F}(p)\right] \\
& =\sqrt{g_{m}}(+i g)(-i)^{2}\left(\frac{v}{c}\right)^{1-\delta_{\mu 0}} \frac{1}{v^{2}} \int \frac{d^{2} \bar{p}_{E} d p_{0}}{(2 \pi)^{3}} \operatorname{Tr}\left[\begin{array}{ll}
\gamma_{\mu} \gamma_{\alpha} & \gamma_{\beta}
\end{array}\right] \frac{(\bar{k}+\bar{p})_{\alpha}(\bar{p})_{\beta}}{\left[x(\bar{k}+\bar{p})^{2}+(1-x) \bar{p}^{2}\right]^{2}}=0 .
\end{aligned}
$$

The momentum part is proportional to $\delta_{\alpha \beta} p^{2}$, while the trace is proportional to the $\epsilon_{\mu \alpha \beta}$ tensor, and so it vanishes. 
c. "1-1” vacuum polarization scalar

$$
\begin{aligned}
\Pi_{m}\left(k_{0}, \mathbf{k}\right) & =\left(\sqrt{g_{m}}\right)^{2} N_{f} \times(-1) \int \frac{d^{2} p_{E} d p_{0}}{(2 \pi)^{3}} \operatorname{Tr}\left[\mathbf{1} S_{F}(k+p) \mathbf{1} S_{F}(p)\right] \\
& =g_{m} \frac{1}{v^{2}} \int \frac{d^{2} \bar{p}_{E} d p_{0}}{(2 \pi)^{3}} \operatorname{Tr}\left[\gamma_{\alpha} \gamma_{\beta}\right] \frac{(\bar{k}+\bar{p})_{\alpha}(\bar{p})_{\beta}}{\left[x(\bar{k}+\bar{p})^{2}+(1-x) \bar{p}^{2}\right]^{2}}=\frac{-|k| g_{m}}{8 v^{2}} .
\end{aligned}
$$

Although nonzero, when connecting external fermion lines, the resulting diagram would be proportional to the number of replicas $n_{R}$ and vanish in the $n_{r} \rightarrow 0$ limit.

\section{d. Effective gauge propagator}

In Coulomb gauge (longitudinal component $a_{L}=0$ ), the kinetic term for the gauge field is

$$
S_{\text {gauge }}=\frac{1}{2} \int d k^{2} d \omega\left(a_{0} \quad a_{T}\right)\left(\begin{array}{cc}
\frac{g^{2}}{16} \frac{k^{2}}{\sqrt{\omega^{2}+v^{2} k^{2}}} & i \kappa|k| \\
i \kappa|k| & w_{x} \frac{k^{2}}{|k|+f(\omega, k)}+\frac{g^{2}}{16} \sqrt{\omega^{2}+v^{2} k^{2}}
\end{array}\right)\left(\begin{array}{l}
a_{0} \\
a_{T}
\end{array}\right),
$$

where $\left(k^{2} \equiv|\mathbf{k}|^{2}\right)$. Recall that $g_{1} \equiv \frac{g^{2}}{16}=\frac{1}{16}$, the effective Coulomb coupling $w_{x} \equiv \frac{+e^{2}}{4 \pi^{2}}$, and $f(k, \omega) \equiv \frac{\sigma_{e} \mathbf{k}^{2}}{|\omega|+D_{e} \mathbf{k}^{2}}$. The transverse component of the gauge field is $a_{T}(k, \omega) \equiv i \hat{k}_{x} a_{y}(k, \omega)-i \hat{k}_{y} a_{x}(k, \omega)$, where $\hat{k}_{j}=k_{j} /|k|$.

When dealing with the gamma matrix contraction in Feynman diagram calculations, we have to write the effective gauge propagator obtained from $S_{\text {gauge }}$ in the $a_{0}, a_{x}$, and $a_{y}$ basis $(i, j=x, y)$ :

$$
\begin{aligned}
& G_{00}=\frac{1}{\mathbf{k}^{2}} \frac{g_{1} \sqrt{\omega^{2}+v^{2} \mathbf{k}^{2}}+F(k, \omega)}{g_{1}^{2}+\kappa^{2}+\frac{g_{1} F(k, \omega)}{\sqrt{\omega^{2}+v^{2} \mathbf{k}^{2}}}}, \\
& G_{0 i}=\frac{\kappa}{\mathbf{k}^{2}} \frac{-\epsilon_{i j} k_{j}}{g_{1}^{2}+\kappa^{2}+\frac{g_{1} F(k, \omega)}{\sqrt{\omega^{2}+v^{2} \mathbf{k}^{2}}}}, \\
& G_{i 0}=-G_{0 i}=\frac{\kappa}{\mathbf{k}^{2}} \frac{+\epsilon_{i j} k_{j}}{g_{1}^{2}+\kappa^{2}+\frac{g_{1} F(k, \omega)}{\sqrt{\omega^{2}+v^{2} \mathbf{k}^{2}}}}, \\
& G_{i j}=\left(\delta_{i j}-\frac{k_{i} k_{j}}{\mathbf{k}^{2}}\right) \frac{\frac{g_{1}}{\sqrt{\omega^{2}+v^{2} \mathbf{k}^{2}}}}{\left[g_{1}^{2}+\kappa^{2}+\frac{g_{1} F(k, \omega)}{\sqrt{\omega^{2}+v^{2} \mathbf{k}^{2}}}\right]} \\
& \text { where } F(k, \omega) \equiv \frac{+e^{2}}{4 \pi^{2}} \frac{|\mathbf{k}|^{2}}{|\mathbf{k}|+f(k, \omega)}=\frac{+e^{2}}{4 \pi^{2}} \frac{|\mathbf{k}|^{2}}{|\mathbf{k}|+\frac{\sigma_{e} \mathbf{k}^{2}}{|\omega|+D_{e} \mathbf{k}^{2}}} \\
& \stackrel{\text { critical limit, } D_{e}=0}{\longrightarrow} \frac{+e^{2}}{4 \pi^{2}} \frac{|\mathbf{k}|^{2}}{|\mathbf{k}|+\frac{\sigma_{e} \mathbf{k}^{2}}{|\omega|}} .
\end{aligned}
$$

\section{Screened disorder $W_{\mu \nu}$}

The disorders $g_{0}$ and $g_{j}$ are screened by the fermion polarization. The Feynman rules in (C2) and (C3) have to be adjusted to account for this screening:

$$
W_{\mu \nu}=W_{\mu \nu}^{(0)}+\phi_{1} W_{\mu \nu}^{(\mathrm{sc})},
$$

where $W_{\mu \nu}^{(0)}=\operatorname{diag}\left(g_{0}, i^{2} g_{j}, i^{2} g_{j}\right)$ is the bare part in (C2) and (C3) and $W_{\mu \nu}^{(\mathrm{sc})}$ is the screening part from the summation of fermion bubbles.

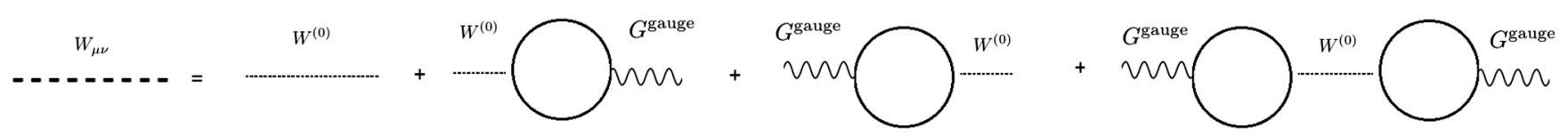

The prefactor $\phi_{1}$ isolates the screened and unscreened contributions: $\phi_{1}=0$ means that disorder screening is ignored; $\phi_{1}=1$ means that disorder screening is included. When disorder connects with the gauge propagator, we should set $\sigma_{e}=0$ before setting $\omega=0$ [due to the presence of the $\delta(\omega)$ ] factor. Otherwise, there is no disorder screening. Note that the vertex factors are included in $W_{\mu \nu}$, so when applying the Feynman rules we only need to multiply by $\gamma_{\mu}$ without any constant or velocity factor. 
We separate the screening part into symmetric and antisymmetric components:

$$
\begin{gathered}
W^{(\mathrm{sc})}=W^{\mathrm{sym}}+W^{\mathrm{as}}, \\
W_{00}^{\mathrm{sym}}=g_{1} \frac{-g_{1} g_{j} \kappa^{2}+g_{0}\left(g_{1}+\overline{w_{x}}\right)\left(g_{1}^{2}+g_{1} \overline{w_{x}}+2 \kappa^{2}\right)}{\left(g_{1}^{2}+g_{1} \overline{w_{x}}+\kappa^{2}\right)^{2}}, \\
W_{i j}^{\mathrm{sym}}=\frac{g_{1}^{2}\left[g_{1}^{2} g_{j}+2 g_{1} g_{j} \overline{w_{x}}-\left(g_{0}-2 g_{j}\right) \kappa^{2}\right]}{\left(g_{1}^{2}+g_{1} \overline{w_{x}}+\kappa^{2}\right)^{2}} \frac{1}{k^{2}}\left(k^{2} \delta_{i j}-k_{i} k_{j}\right), \\
W_{0 i}^{\mathrm{as}}=\frac{g_{1} \kappa\left[g_{1} g_{j} \overline{w_{x}}+\left(-g_{0}+g_{j}\right) \kappa^{2}\right]}{\left[g_{1}^{2}+g_{1} \overline{w_{x}}+\kappa^{2}\right]^{2}} \frac{-\epsilon_{i j} k_{j}}{k} .
\end{gathered}
$$

Other components of $W^{(\mathrm{sc})}$ not included above vanish.

\section{Effective gauge disorder $D_{\mu v}^{\text {dis }}$}

The expressions in (C4) and (C5) are two-point vertex rules: each side of the vertex connects with the dressed propagator found in (C14)-(C16). The effective gauge disorder is defined by

$$
D_{\mu \nu}^{\text {dis }}=G_{\mu \alpha} D_{\alpha \beta}^{0, \text { dis }} G_{\beta \nu},
$$

where $D_{00}^{0, \text { dis }}=-\Delta_{j} \boldsymbol{k}^{2}$ defined in (C5), $D_{i j}^{0, \text { dis }}=\Delta_{0}\left(\delta_{i j} \boldsymbol{k}^{2}-k_{i} k_{j}\right)$ defined in $(\mathrm{C} 4)$, and $D_{0 i}^{0, \text { dis }}=D_{i 0}^{0 \text {,dis }}=0$. We decompose $D_{\mu \nu}^{\text {dis }}$ into symmetric and antisymmetric components:

$$
\begin{gathered}
D_{\mu \nu}^{\mathrm{dis}}=D_{\mu \nu}^{\mathrm{S}}+D_{\mu \nu}^{\mathrm{AS}} \\
D_{00}^{\mathrm{S}}=-\frac{g_{1}^{2} v^{2} \Delta_{j}+2 g_{1} v^{2} \overline{w_{x}} \Delta_{j}+v^{2} \bar{w}_{x}^{2} \Delta_{j}+\Delta_{0} \kappa^{2}}{\left(g_{1}^{2}+g_{1} \overline{w_{x}}+\kappa^{2}\right)^{2}}, \\
D_{i j}^{\mathrm{S}}=\frac{\left(g 1^{2} \Delta_{0}+v^{2} \Delta_{j} \kappa^{2}\right)}{v^{2}\left(g_{1}^{2}+g_{1} \overline{w_{x}}+\kappa^{2}\right)^{2}} \frac{k^{2} \delta_{i j}-k_{i} k_{j}}{k^{2}}, \\
D_{0 i}^{\mathrm{AS}}=\frac{\kappa\left(-g_{1} \Delta_{0}+g_{1} v^{2} \Delta_{j}+v^{2} \overline{w_{x}} \Delta_{j}\right)}{v\left(g_{1}^{2}+g_{1} \overline{w_{x}}+\kappa^{2}\right)^{2}} \frac{\epsilon_{j}}{k} .
\end{gathered}
$$

Components of $D^{\text {dis }}$ not listed above are zero.

Since $G_{\mu \nu}$ is constructed by the random-phase-approximation sum of fermion loops, $G_{\mu \nu}$ can no longer connect any more fermion loops. Consequently, $D_{\mu \nu}^{\text {dis }}$ does not include any fermion loops. Note that $D_{\mu \nu}^{\text {dis }}$ generates $\frac{\Delta_{0, j}}{N_{f}}$. This disorder renormalizes $\Delta_{0}$ and $\Delta_{j}$ at three-loop order.

\section{APPENDIX D: FERMION SELF-ENERGY}

\section{Self-energy: Screened disorder correction $W_{\mu \nu}$}

$$
\begin{aligned}
\Sigma_{d}\left(p_{0}, \boldsymbol{p}\right)= & \int \frac{d^{2} \boldsymbol{k}}{(2 \pi)^{2}}\left(\mathbf{1}_{2 \times 2}\right) S_{F}\left(p_{0}-0, \boldsymbol{p}-\boldsymbol{k}\right)\left(\mathbf{1}_{2 \times 2}\right) g_{m}+\int \frac{d^{2} \boldsymbol{k}}{(2 \pi)^{2}} \gamma_{\nu} S_{F}\left(p_{0}-0, \boldsymbol{p}-\boldsymbol{k}\right) \gamma_{\mu} W_{\mu \nu}(\boldsymbol{k}) \\
= & \int \frac{d^{2} \boldsymbol{k}}{(2 \pi)^{2}}\left(\mathbf{1}_{2 \times 2}\right) S_{F}\left(p_{0}-0, \boldsymbol{p}-\boldsymbol{k}\right)\left(\mathbf{1}_{2 \times 2}\right) g_{m}+\int \frac{d^{2} \boldsymbol{k}}{(2 \pi)^{2}} \gamma_{\nu}\left[(+i) \frac{\gamma_{0} p_{0}+v(\boldsymbol{p}-\boldsymbol{k})_{i} \gamma_{i}}{p_{0}^{2}+v^{2}(\boldsymbol{p}-\boldsymbol{k})^{2}}\right] \gamma_{\mu} W_{\mu v}(\boldsymbol{k}) \\
= & \frac{+i}{v^{2}} \frac{p_{0} \gamma_{0}}{2 \pi \epsilon}\left[g_{m}\right]+\int \frac{d^{2} \boldsymbol{k}}{(2 \pi)^{2}} \gamma_{\nu}\left[(+i) \frac{\gamma_{0} p_{0}+v(\boldsymbol{p}-\boldsymbol{k})_{i} \gamma_{i}}{p_{0}^{2}+v^{2}(\boldsymbol{p}-\boldsymbol{k})^{2}}\right] \gamma_{\mu} W_{\mu \nu}(\boldsymbol{k}) \\
= & \frac{+i p_{0} \gamma_{0}}{2 \pi \epsilon v^{2}} g_{m}+\frac{+i p_{0} \gamma_{0}}{2 \pi \epsilon v^{2}}\left(g_{0}+2 g_{j}\right)+\phi_{1}\left[\frac{g_{1}^{2} \overline{w_{x}}\left(g_{1} \overline{g_{j}}-g_{1} \overline{g_{0}}-\overline{g_{0}} \overline{w_{x}}\right)}{\left(g_{1}^{2}+g_{1} \overline{w_{x}}+\kappa^{2}\right)^{2}}\right. \\
& \left.+\frac{g_{1}\left(g_{1} \overline{g_{j}}+g_{1} \overline{g_{0}}+2 \overline{g_{0}} \overline{w_{x}}\right)}{g_{1}^{2}+g_{1} \overline{w_{x}}+\kappa^{2}}\right] \frac{-i}{\epsilon} p_{0} \gamma_{0}+\phi_{1} \frac{(-1) g_{1}^{2}\left[g_{1}^{2} \overline{g_{j}}+2 g_{1} \overline{g_{j}} \overline{w_{x}}-\left(\overline{g_{0}}-2 \overline{g_{j}}\right) \kappa^{2}\right]}{\left(g_{1}^{2}+g_{1} \overline{w_{x}}+\kappa^{2}\right)^{2}} \frac{-i}{\epsilon} p_{j} \gamma_{j} .
\end{aligned}
$$




\section{Self-energy: Gauge correction}

Only the symmetric part of the gauge propagator produces a divergence at $\mathcal{O}\left(\frac{1}{N_{f}}\right)$ :

$$
\begin{gathered}
\Sigma_{g}\left(p_{0}, \boldsymbol{p}\right)=\left(\frac{-i g}{\sqrt{N_{f}}}\right)^{2}\left(\frac{v}{c}\right)^{2-\delta_{\mu 0}-\delta_{\nu 0}} \int \frac{d^{3} k}{(2 \pi)^{3}} \gamma_{\nu} S_{F}(p-k) \gamma_{\mu} G_{\mu \nu}\left(k_{0}, \boldsymbol{k}\right) \\
=\frac{-g^{2}}{N_{f}}(+i)\left(\frac{v}{c}\right)^{2-\delta_{\mu 0}-\delta_{\nu 0}} \frac{1}{v^{2}} \int \frac{d^{2} \bar{k} d k_{0}}{(2 \pi)^{3}}\left[\gamma_{\nu} \frac{\left(p_{0}-k_{0}\right) \gamma_{0}+(\bar{p}-\bar{k})_{a} \gamma_{a}}{\left(p_{0}-k_{0}\right)^{2}+(\bar{p}-\bar{k})^{2}} \gamma_{\mu}\right] G_{\mu \nu}\left(k_{0}, \bar{k}\right) .
\end{gathered}
$$

Carrying out the momentum integral and setting $c=1$,

$$
\begin{aligned}
-\Sigma_{g}\left(p_{0}, \boldsymbol{p}\right)= & \int_{-\infty}^{\infty} d z \frac{i g^{2}\left\{\left(v^{2}-z^{2}\right) g_{1} z^{2} \sigma_{e}+\left[g_{1} z^{2} v^{2}-g_{1} z^{4} \times \xi+w_{x} \sqrt{v^{2}+z^{2}}\left(v^{2}-z^{2}\right)\right]|z|\right\}\left(p_{0} \gamma_{0}\right)}{4 \epsilon N_{f} \pi^{2}\left(v^{2}+z^{2}\right)^{2}\left[\sqrt{v^{2}+z^{2}}\left(g_{1}^{2}+\kappa^{2}\right) \sigma_{e}+|z|\left(\left(g_{1}^{2}+\kappa^{2}\right) \sqrt{v^{2}+z^{2}}+g_{1} w_{x}\right)\right]} \\
& +\int_{-\infty}^{\infty} d z \frac{i g^{2}\left\{g_{1}\left(v^{4}-v^{2} z^{2}-z^{4}\right) \sigma_{e}-\left[w_{x} z^{2} \sqrt{v^{2}+z^{2}}+g_{1}\left(-v^{4}+v^{2} z^{2}+z^{4} \times \xi\right)\right]|z|\right\}\left(v p_{j} \gamma_{j}\right)}{4 \epsilon N_{f} \pi^{2}\left(v^{2}+z^{2}\right)^{2}\left[\sqrt{v^{2}+z^{2}}\left(g_{1}^{2}+\kappa^{2}\right) \sigma_{e}+|z|\left(\left(g_{1}^{2}+\kappa^{2}\right) \sqrt{v^{2}+z^{2}}+g_{1} w_{x}\right)\right]}
\end{aligned}
$$

To obtain the above expression, we first perform a gradient expansion of $\Sigma\left(p_{0}, \boldsymbol{p}\right)$ around $p_{0}=\boldsymbol{p}=0$. Next, focus on the linear term in $p_{0}$ and $\boldsymbol{p}$ and replace the frequency integral $k_{0} \rightarrow z|\boldsymbol{k}|$. When the $2 \mathrm{D}$ spatial momentum integral is done, the result is the expression shown above. The above expression is integrable only at $\sigma_{e}=0$. The $\xi$ term is a divergent integral that arises from the choice of Coulomb gauge. Physical observables are free from any $\xi$ dependence.

\section{Self-energy: Effective gauge disorder $D_{\mu \nu}^{\text {dis }}, \mathcal{O}\left(\frac{\Delta_{X}}{N_{f}}\right)$}

$$
\begin{aligned}
\Sigma_{b}\left(p_{0}, \boldsymbol{p}\right) & =\left(\frac{-i g}{\sqrt{N_{f}}}\right)^{2}\left(\frac{v}{c}\right)^{2-\delta_{\mu 0}-\delta_{\nu 0}} \int \frac{d^{2} \boldsymbol{k}}{(2 \pi)^{2}} \gamma_{\nu} S_{F}\left(p_{0}-0, \boldsymbol{p}-\boldsymbol{k}\right) \gamma_{\mu} D_{\mu \nu}^{\mathrm{dis}}(\boldsymbol{k}) \\
& =\frac{+i\left(\Delta_{0}+v^{2} \Delta_{j}\right)}{2 N_{f} \pi v^{2} \epsilon\left(g_{1}^{2}+\kappa^{2}\right)} \gamma_{0} p_{0}+\frac{-i\left(g_{1}^{2} \Delta_{0}+\kappa^{2} v^{2} \Delta_{j}\right)}{2 N_{f} \pi v^{2} \epsilon\left(g_{1}^{2}+\kappa^{2}\right)^{2}}\left(v \gamma_{j} p_{j}\right) .
\end{aligned}
$$

\section{APPENDIX E: THREE-POINT VERTEX $\bar{u}(q) \delta \Gamma^{\mu} u(p)$}
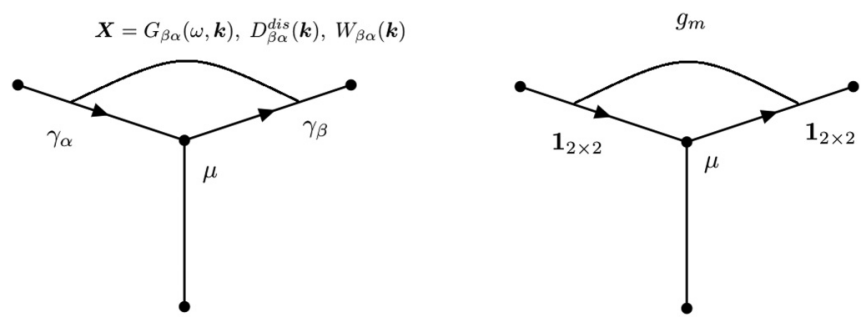

\section{1. $\Gamma^{\mu}$ : Gauge correction}

$$
\begin{aligned}
\Gamma_{1}^{\mu} & =\left(\frac{-i g}{\sqrt{N_{f}}}\right)^{3}\left(\frac{v}{c}\right)^{3-\delta_{\alpha 0}-\delta_{\beta 0}-\delta_{\mu 0}} \int \frac{d^{2} \boldsymbol{k} d \omega}{(2 \pi)^{3}} \gamma_{\alpha} S_{F}(q-k) \gamma_{\mu} S_{F}(p-k) \gamma_{\beta} G_{\beta \alpha}(k, \omega) \\
& =\left(\frac{-i g}{\sqrt{N_{f}}}\right)^{3}\left(\frac{v}{c}\right)^{3-\delta_{\alpha 0}-\delta_{\beta 0}-\delta_{\mu 0}} \frac{1}{v^{2}} \int \frac{d^{2} \overline{\boldsymbol{k}} d \omega}{(2 \pi)^{3}} \gamma_{\alpha}(+i) \frac{\gamma_{0}\left(q_{0}-k_{0}\right)+\gamma_{c}(\bar{q}-\bar{k})_{c}}{\left(q_{0}-k_{0}\right)^{2}+(\bar{q}-\bar{k})^{2}} \gamma_{\mu}(+i) \frac{\gamma_{0}\left(p_{0}-k_{0}\right)+\gamma_{d}(\bar{p}-\bar{k})_{d}}{\left(p_{0}-k_{0}\right)^{2}+(\bar{p}-\bar{k})^{2}} \gamma_{\beta} G_{\beta \alpha}(k, \omega) .
\end{aligned}
$$


To isolate the divergent part, one can set the external momentum $p=q=0$. Following the same steps we used in the self-energy diagram evaluation, we obtain

$$
\begin{aligned}
\Gamma_{1}^{\mu}= & \int_{-\infty}^{\infty} d z \frac{-i g}{\sqrt{N_{f}}} \frac{\left(-g^{2}\right)\left\{\left(v^{2}-z^{2}\right) g_{1} z^{2} \sigma_{e}+\left[g_{1} z^{2} v^{2}-g_{1} z^{4} \times \xi+w_{x} \sqrt{v^{2}+z^{2}}\left(v^{2}-z^{2}\right)\right]|z|\right\}}{\pi^{2}\left(v^{2}+z^{2}\right)^{2}\left[\sqrt{v^{2}+z^{2}}\left(g_{1}^{2}+\kappa^{2}\right) \sigma_{e}+|z|\left(\left(g_{1}^{2}+\kappa^{2}\right) \sqrt{v^{2}+z^{2}}+g_{1} w_{x}\right)\right]}\left(\gamma_{0}\right) \\
& +\int_{-\infty}^{\infty} d z \frac{-i g}{\sqrt{N_{f}}} \frac{v}{1} \frac{\left(-g^{2}\right)\left(g_{1}\left(v^{4}-v^{2} z^{2}-z^{4}\right) \sigma_{e}-\left[w_{x} z^{2} \sqrt{v^{2}+z^{2}}+g_{1}\left(-v^{4}+v^{2} z^{2}+z^{4} \times \xi\right)\right]|z|\right)}{4 \pi^{2}\left(v^{2}+z^{2}\right)^{2}\left[\sqrt{v^{2}+z^{2}}\left(g_{1}^{2}+\kappa^{2}\right) \sigma_{e}+|z|\left(\left(g_{1}^{2}+\kappa^{2}\right) \sqrt{v^{2}+z^{2}}+g_{1} w_{x}\right)\right]}\left(\gamma_{j}\right) .
\end{aligned}
$$

As before, $\xi$ labels the divergent part. Gauge invariance is easy to check by comparing with Eq. (D5): $\Gamma_{1}^{t}=\frac{-g}{\sqrt{N_{f}}} \frac{\partial \Sigma_{g}}{\partial p_{0}}$ and $\Gamma_{1}^{j}=$ $\frac{-g}{\sqrt{N_{f}}} \frac{\partial \Sigma_{g}}{\partial p_{j}}$.

\section{2. $\Gamma^{\mu}$ : Effective gauge disorder correction}

$$
\begin{gathered}
\Gamma_{2}^{\mu}=\left(\frac{-i g}{\sqrt{N_{f}}}\right)^{3}\left(\frac{v}{c}\right)^{3-\delta_{\alpha 0}-\delta_{\beta 0}-\delta_{\mu 0}} \int \frac{d^{2} \boldsymbol{k}}{(2 \pi)^{2}} \gamma_{\alpha} S_{F}(q-k) \gamma_{\mu} S_{F}(p-k) \gamma_{\beta} D_{\beta \alpha}^{\mathrm{dis}}(\boldsymbol{k}) \\
=\frac{-i\left(\Delta_{0}+v^{2} \Delta_{j}\right)}{2 N_{f}^{3 / 2} \pi v^{2} \epsilon\left(g_{1}^{2}+\kappa^{2}\right)} \gamma^{0}+\frac{i\left(g_{1}^{2} \Delta_{0}+v^{2} \kappa^{2} \Delta_{j}\right)}{2 N_{f}^{3 / 2} \pi v \epsilon\left(g_{1}^{2}+\kappa^{2}\right)^{2}} \gamma^{j} . \\
\text { 3. } \boldsymbol{\Gamma}^{\mu} \text { : Screened disorder correction } \boldsymbol{W}_{\mu \nu} \\
\Gamma_{3}^{\mu}=\left(\frac{-i g}{\sqrt{N_{f}}}\right)\left(\frac{v}{c}\right)^{1-\delta_{\mu 0}} \times \int \frac{d^{2} \boldsymbol{k}}{(2 \pi)^{2}} \gamma_{\alpha} S_{F}(q-k) \gamma_{\mu} S_{F}(p-k) \gamma_{\beta} W_{\beta \alpha} \\
=\frac{-i g}{\sqrt{N_{f}}} \frac{1}{\epsilon}\left(\frac{g_{0}+2 g_{j}}{2 \pi v^{2}}+\phi_{1}\left[\frac{g_{1}^{2} \overline{w_{x}}\left(-g_{1} \overline{g_{j}}+g_{1} \overline{g_{0}}+\overline{g_{0}} \overline{w_{x}}\right)}{\left(g_{1}^{2}+g_{1} \overline{w_{x}}+\kappa^{2}\right)^{2}}-\frac{g_{1}\left(g_{1} \overline{g_{j}}+g_{1} \overline{g_{0}}+2 \overline{g_{0}} \overline{w_{x}}\right)}{g_{1}^{2}+g_{1} \overline{w_{x}}+\kappa^{2}}\right]\right) \gamma^{0} \\
+\frac{-i g}{\sqrt{N_{f}}} \phi_{1} \frac{1}{\epsilon}\left(\frac{g_{1}^{2}\left[g_{1}^{2} \overline{g_{j}}+2 g_{1} \overline{g_{j}} \overline{w_{x}}-\left(\overline{g_{0}}-2 \overline{g_{j}}\right) \kappa^{2}\right]}{\left(g_{1}^{2}+g_{1} \overline{w_{x}}+\kappa^{2}\right)^{2}}\right) \gamma^{j} .
\end{gathered}
$$

\section{4. $\Gamma^{\mu}$ : Random mass correction $g_{m}$}

$$
\Gamma_{4}^{\mu}=\left(\frac{-i g}{\sqrt{N_{f}}}\right)\left(\frac{v}{c}\right)^{1-\delta_{\mu 0}} \times \int \frac{d^{2} \boldsymbol{k}}{(2 \pi)^{2}} \mathbf{1} S_{F}(q-k) \gamma_{\mu} S_{F}(p-k) \mathbf{1} g_{m}=\frac{-i g}{\sqrt{N_{f}}} \frac{g_{m}}{2 \pi v^{2} \epsilon} \gamma^{0}+0 \gamma^{j}
$$

APPENDIX F: THREE-POINT VERTEX $\bar{u}(q) 1_{2 \times 2} u(p)$

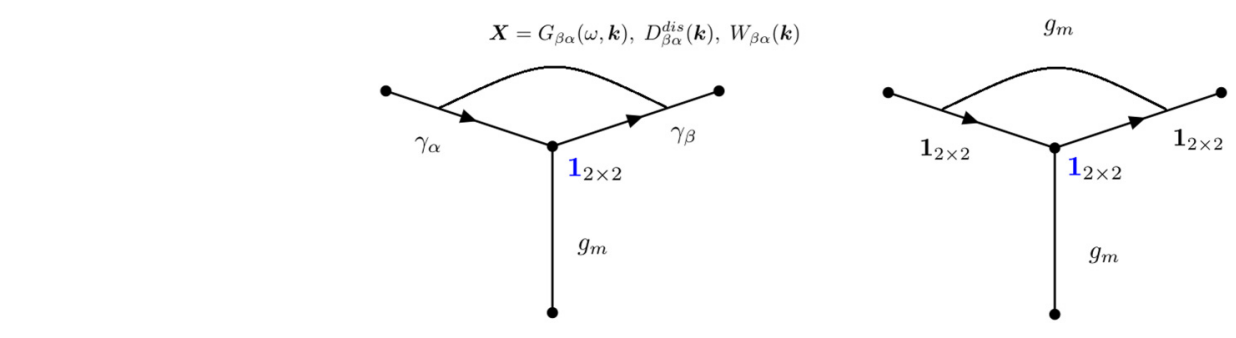

1. $1_{2 \times 2}$ : Gauge correction

$$
\Gamma_{1}^{m}=\left(\frac{-i g}{\sqrt{N_{f}}}\right)^{2}\left(\frac{v}{c}\right)^{2-\delta_{\alpha 0}-\delta_{\beta 0}} \frac{1}{v^{2}}(+i)^{2} \int \frac{d^{2} \bar{k} d \omega}{(2 \pi)^{3}} \gamma_{\alpha} \frac{\gamma_{0}\left(q_{0}-\omega\right)+\gamma_{c}(\bar{q}-\bar{k})_{c}}{\left(q_{0}-\omega\right)^{2}+(\bar{q}-\bar{k})^{2}} \mathbf{1} \frac{\gamma_{0}\left(p_{0}-\omega\right)+\gamma_{d}(\bar{p}-\bar{k})_{d}}{\left(p_{0}-\omega\right)^{2}+(\bar{p}-\bar{k})^{2}} \gamma_{\beta} G_{\alpha \beta},
$$




$$
\begin{aligned}
\Gamma_{1}^{m} & =\int_{-\infty}^{\infty} d z \frac{\left(v^{2}+z^{2}\right) g^{2}\left[g_{1}\left(2 v^{2}+z^{2}\right) \sigma_{e}+w_{x} \sqrt{v^{2}+z^{2}}+g_{1}\left(2 v^{2}+z^{2}\right)|z|\right]}{4 \epsilon N_{f} \pi^{2}\left(v^{2}+z^{2}\right)^{2}\left[\sqrt{v^{2}+z^{2}}\left(g_{1}^{2}+\kappa^{2}\right) \sigma_{e}+|z|\left(\left(g_{1}^{2}+\kappa^{2}\right) \sqrt{v^{2}+z^{2}}+g_{1} w_{x}\right)\right]} \mathbf{1}_{2 \times 2} \\
& =\int_{-\infty}^{\infty} d z \frac{g^{2}\left[g_{1}\left(2 v^{2}+z^{2}\right)\left(v^{2}+z^{2}\right) \sigma_{e}+w_{x}\left(v^{2}+z^{2}\right) \sqrt{v^{2}+z^{2}}+g_{1}\left(2 v^{4}+3 z^{2} v^{2}+\xi z^{4}\right)|z|\right]}{4 \epsilon N_{f} \pi^{2}\left(v^{2}+z^{2}\right)^{2}\left[\sqrt{v^{2}+z^{2}}\left(g_{1}^{2}+\kappa^{2}\right) \sigma_{e}+|z|\left(\left(g_{1}^{2}+\kappa^{2}\right) \sqrt{v^{2}+z^{2}}+g_{1} w_{x}\right)\right]} \mathbf{1}_{2 \times 2} .
\end{aligned}
$$

\section{2. $1_{2 \times 2}$ : Effective gauge disorder correction}

$$
\begin{aligned}
\Gamma_{2}^{m} & =\left(\frac{-i g}{\sqrt{N_{f}}}\right)^{2}\left(\frac{v}{c}\right)^{2-\delta_{\alpha 0}-\delta_{\beta 0}} \int \frac{d^{2} \boldsymbol{k}}{(2 \pi)^{2}} \gamma_{\alpha} S_{F}(q-k) \mathbf{1} S_{F}(p-k) \gamma_{\beta} D_{\beta \alpha}^{\mathrm{dis}}(\boldsymbol{k}) \\
& =\frac{\left(\Delta_{0}-v^{2} \Delta_{j}\right)\left(g_{1}^{2}-c^{4} \kappa^{2}\right)}{2 \pi N_{f} v^{2} \epsilon\left(g_{1}^{2}+c^{4} \kappa^{2}\right)^{2}} \mathbf{1}_{2 \times 2 .} .
\end{aligned}
$$

\section{3. $1_{2 \times 2}$ : Screened disorder correction $W_{\mu v}$}

$$
\begin{aligned}
\Gamma_{3}^{m} & =\int \frac{d^{2} \boldsymbol{k}}{(2 \pi)^{2}} \gamma_{\alpha} S_{F}(q-k) \mathbf{1} S_{F}(p-k) \gamma_{\beta} W_{\beta \alpha} \\
& =\left[\frac{-g_{0}+2 g_{j}}{2 \pi v^{2} \epsilon}+\phi_{1} \frac{1}{\epsilon}\left(-\frac{g_{1}^{2}\left(2 g_{1}+\overline{w_{x}}\right)\left(-g_{1} g_{j}+\overline{g_{0}} g_{1}+\overline{g_{0}} \overline{w_{x}}\right.}{\left(g_{1}^{2}+g_{1} \overline{w_{x}}+\kappa^{2}\right)^{2}}+\frac{g_{1}\left(3 \overline{g_{0}} g_{1}-3 g_{1} \overline{g_{j}}+2 \overline{g_{0}} \overline{w_{x}}\right)}{g_{1}^{2}+g_{1} \overline{w_{x}}+\kappa^{2}}\right)\right] \mathbf{1}_{2 \times 2} .
\end{aligned}
$$

\section{4. $1_{2 \times 2}$ : Random mass correction $g_{m}$}

$$
\Gamma_{4}^{m}=\left(\frac{-i g}{\sqrt{N_{f}}}\right) \times \int \frac{d^{2} \boldsymbol{k}}{(2 \pi)^{2}} \mathbf{1} S_{F}(q-k) \mathbf{1} S_{F}(p-k) \mathbf{1} g_{m}=\frac{-g_{m}}{2 \pi v^{2} \epsilon} \mathbf{1}_{2 \times 2} .
$$

\section{APPENDIX G: FOUR-POINT FERMION-FERMION INTERACTION}

Define

$$
H_{\mu \nu} \equiv D_{\mu \nu}^{\mathrm{dis}}\left(\frac{-i g}{\sqrt{N_{f}}}\right)^{2}\left(\frac{v}{c}\right)^{2-\delta_{\mu 0}-\delta_{\nu 0}} .
$$

Note that $W_{\mu \nu} \sim \mathcal{O}\left(\frac{1}{N_{f}^{0}}\right)$ and $H_{\mu \nu} \sim \mathcal{O}\left(\frac{1}{N_{f}}\right)$. Take the external three momenta to be $p_{1}, p_{2}, p_{3}, p_{4}$, where $p_{i}=\left(\omega_{i}, \boldsymbol{p}_{i}\right)$. Schematically, the interaction has the form $\left[\bar{\psi}\left(p_{3}\right) \ldots \psi\left(p_{1}\right)\right]\left[\bar{\psi}\left(p_{4}\right) . . \psi\left(p_{2}\right)\right]$. Define

$$
\begin{gathered}
\Gamma_{A} \equiv\left(\gamma_{7}, \gamma_{0},+\gamma_{x},+\gamma_{y}\right), \quad \gamma_{7} \equiv \mathbf{1}_{2 \times 2} \\
T^{A_{1}}=\left(\mathbf{1} g_{m}, W_{\mu \nu}, H_{\mu \nu}\right), \quad \tilde{T}^{A_{1}}=\left(\mathbf{1} g_{m}, W_{\mu \nu}^{(0)}, H_{\mu \nu}\right), \\
W_{\mu \nu}^{(0)}=\operatorname{diag}\left(g_{0}, i^{2} g_{j}, i^{2} g_{j}\right) .
\end{gathered}
$$

We use $A, B, C, D=\{1,2,3,4\}$ indices to label $\mathbf{1}, \gamma_{0}, \gamma_{x}, \gamma_{y}$ and number subscripts, e.g., $A_{1}$ and $A_{2}$, to label which interaction we choose: $A_{1}=1$ for the $g_{m}$ interaction; $A_{1}=2$ for the $W_{\mu \nu}$ interaction; $A_{1}=3$ for the $H_{\mu \nu}$ interaction.

The diagrams below correspond to the following expressions:

$$
\begin{aligned}
B_{1} & =\int \frac{d^{2} k}{(2 \pi)^{2}} \bar{\psi}\left(p_{3}\right) \Gamma_{B} S_{F}\left(p_{1}+k\right) \Gamma_{A} \psi\left(p_{1}\right) \bar{\psi}\left(p_{4}\right) \Gamma_{D} S_{F}\left(p_{2}-k\right) \Gamma_{C} \psi\left(p_{2}\right) T_{C A}^{A_{1}}(\boldsymbol{k}, \omega=0) T_{B D}^{A_{2}}\left(p_{1}+k-p_{3}, \omega=0\right), \\
B_{2} & =\int \frac{d^{2} k}{(2 \pi)^{2}} \bar{\psi}\left(p_{3}\right) \Gamma_{B} S_{F}\left(p_{1}+k\right) \Gamma_{A} \psi\left(p_{1}\right) \bar{\psi}\left(p_{4}\right) \Gamma_{C} S_{F}\left(p_{4}+k\right) \Gamma_{D} \psi\left(p_{2}\right) T_{C A}^{A_{1}}(\boldsymbol{k}, \omega=0) T_{B D}^{A_{2}}\left(p_{1}-p_{3}+k, \omega=0\right), \\
B_{3} & =\int \frac{d^{2} k}{(2 \pi)^{2}} \bar{\psi}\left(p_{3}\right) \Gamma_{D} S_{F}\left(p_{3}-k\right) \Gamma_{A} S_{F}\left(p_{1}-k\right) \Gamma_{B} \psi\left(p_{1}\right) T_{B D}^{A_{2}}(\boldsymbol{k}, \omega=0), \bar{\psi}\left(p_{4}\right) \Gamma_{C} \psi\left(p_{2}\right) \tilde{T}_{A C}^{A_{1}}\left(p_{3}-p_{1}, \omega=0\right), \\
B_{4} & =\int \frac{d^{2} k}{(2 \pi)^{2}} \bar{\psi}\left(p_{3}\right) \Gamma_{A} \psi\left(p_{1}\right) \tilde{T}_{A C}^{A_{1}}\left(p_{3}-p_{1}, \omega=0\right) \bar{\psi}\left(p_{4}\right) \Gamma_{D} S_{F}\left(p_{4}-k\right) \Gamma_{C} S_{F}\left(p_{2}-k\right) \Gamma_{B} \psi\left(p_{2}\right) T_{B D}^{A_{2}}(k, \omega=0) .
\end{aligned}
$$




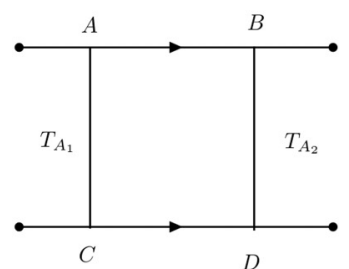

$B_{1}$

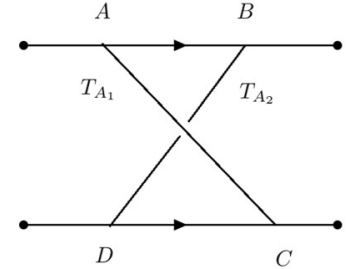

$B_{2}$

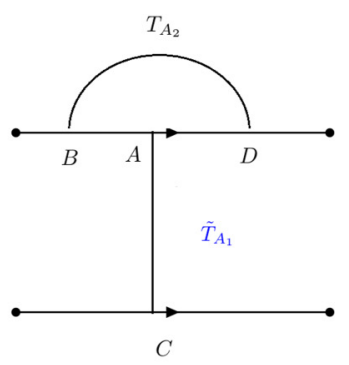

$B_{3}$

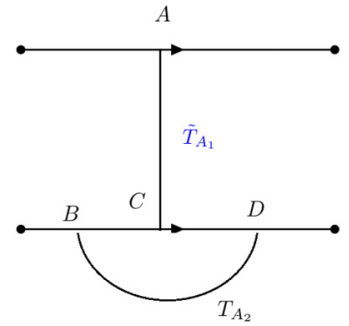

$B_{4}$

For diagrams $B_{3}$ and $B_{4}$, the $T_{A 1}$ vertex is undressed, i.e., $W_{\mu \nu}^{0}$, which is directly related to the random coupling being renormalized.

\section{Four-point interaction: Boxes $B_{3}$ and $B_{4}$}

Diagrams of type $B_{3}$ and $B_{4}$ can be directly obtained from the three-point vertex corrections in Appendices $\mathrm{E}$ and $\mathrm{F}$ with symmetry factor 2 (counting upper or lower vertices), so we do not have to recompute them here. The terms in $\Gamma^{\mu}$ renormalize $g_{0}$ and $g_{j}$ and the terms in $\Gamma^{m}$ renormalize $g_{m}$.

\section{Four-point fermion interaction: Boxes $B_{1}$ and $B_{2}$}

Diagrams for boxes $B_{1}$ and $B_{2}$ are presented below.
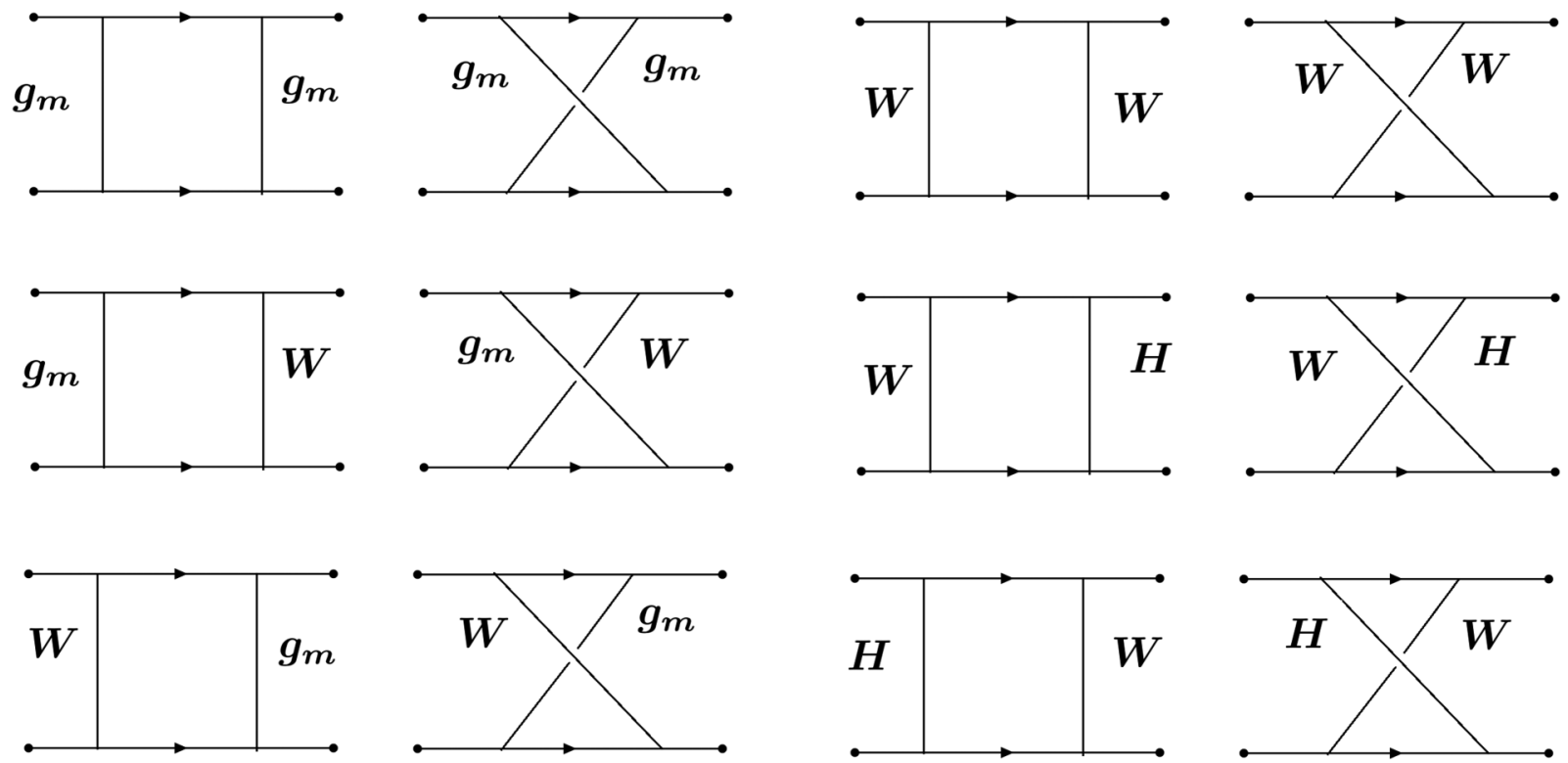

The $W$ - $H$ diagrams are $\mathcal{O}\left(\frac{\Delta_{X} g_{X}}{N_{f}}\right)$. The $H-H$ diagrams are $\mathcal{O}\left(\frac{\Delta_{X}^{2}}{N_{f}^{2}}\right)$.

For each interaction, $(\bar{\psi} \psi)(\bar{\psi} \psi),\left(\bar{\psi} \gamma_{0} \psi\right)\left(\bar{\psi} \gamma_{0} \psi\right),\left(\bar{\psi} i \gamma_{j} \psi\right)\left(\bar{\psi} i \gamma_{j} \psi\right)$, we sum all these diagrams with the help of computer software to $\mathcal{O}\left(g_{X}^{2}, \frac{g_{X}}{N_{f}}\right)$. The contributions from diagrams $B_{1}$ and $B_{2}$ are the following:

$$
\begin{aligned}
\operatorname{Box}_{11}= & {\left[\frac{2 g_{0} g_{j}}{\pi v^{2} \epsilon g_{m}}+\phi_{1}\left(-4 g_{1}\right) \frac{g_{1} \overline{g_{0}} \overline{g_{j}}\left(2 g_{1}^{2}+4 g_{1} \overline{w_{x}}+{\overline{w_{x}}}^{2}\right)-\kappa^{2}\left[\bar{g}_{0}^{2} g_{1}+g_{1}{\overline{g_{j}}}^{2}-2 \overline{g_{0}} \overline{g_{j}}\left(2 g_{1}+\overline{w_{x}}\right)\right]}{\epsilon\left(g_{1}^{2}+g_{1} \overline{w_{x}}+\kappa^{2}\right)^{2}}\right.} \\
& -\frac{\phi_{1}^{2}}{\epsilon}\left(\frac{4 g_{1}^{2}{\overline{g_{j}}}^{2} \kappa^{2}\left[g_{1}^{2}\left(g_{1}^{2}+2 g_{1} \overline{w_{x}}-{\overline{w_{x}}}^{2}\right)+2 g_{1}\left(g_{1}-\overline{w_{x}}\right) \kappa^{2}-\kappa^{4}\right]}{\overline{g_{m}}\left[g_{1}^{2}+g_{1} \overline{w_{x}}+\kappa^{2}\right]^{4}}\right. \\
& +\frac{4 g_{1}^{2} \bar{g}_{0}^{2} \kappa^{2}\left[g_{1}^{2}\left(g_{1}^{2}+2 g_{1} \overline{w_{x}}+{\overline{w_{x}}}^{2}\right)+2 g_{1}\left(g_{1}+\overline{w_{x}}\right) \kappa^{2}-\kappa^{4}\right]}{\overline{g_{m}}\left[g_{1}^{2}+g_{1} \overline{w_{x}}+\kappa^{2}\right]^{4}}
\end{aligned}
$$




$$
\begin{aligned}
& \left.\left.+\frac{4 \overline{g_{0}} \overline{g_{j}} g_{1}^{2}\left[-g_{1}^{3}\left(g_{1}+\overline{w_{x}}\right)^{2}\left(g_{1}+2 \overline{w_{x}}\right)-2 g_{1}^{2}\left(g_{1}+\overline{w_{x}}\right)\left(2 g_{1}+3 \overline{w_{x}}\right) \kappa^{2}-g_{1}\left(5 g_{1}+2 \overline{w_{x}}\right) \kappa^{4}+2 \kappa^{6}\right]}{\overline{g_{m}}\left[g_{1}^{2}+g_{1} \overline{w_{x}}+\kappa^{2}\right]^{4}}\right)\right] \\
& \times(\bar{\psi} \mathbf{1} \psi)(\bar{\psi} \mathbf{1} \psi), \\
\operatorname{Box}_{\gamma_{0} \gamma_{0}}= & {\left[\frac{2 g_{j} g_{m}}{\pi v^{2} \epsilon g_{0}}-\phi_{1} \frac{4 g_{1}^{2} \overline{g_{m}}\left[g_{1}^{2} \overline{g_{j}}+2 g_{1} \overline{g_{j}} \overline{w_{x}}-\left(\overline{g_{0}}-2 \overline{g_{j}}\right) \kappa^{2}\right]}{\overline{g_{0}} \epsilon\left[g_{1}^{2}+g_{1} \overline{w_{x}}+\kappa^{2}\right]^{2}}\right]\left(\bar{\psi} \gamma_{0} \psi\right)\left(\bar{\psi} \gamma_{0} \psi\right), } \\
\operatorname{Box}_{\gamma_{j} \gamma_{j}}= & {\left[\frac{2 g_{0} g_{m}}{\pi v^{2} \epsilon g_{j}}-\phi_{1} \frac{g_{1} \overline{g_{m}}\left[-g_{1} \overline{g_{j}} \kappa^{2}+\overline{g_{0}}\left(g_{1}^{3}+2 g_{1}^{2} \overline{w_{x}}+2 \overline{w_{x}} \kappa^{2}\right)+g_{1}\left({\overline{w_{x}}}^{2}+2 \kappa^{2}\right)\right]}{\overline{g_{j}} \epsilon\left[g_{1}^{2}+g_{1} \overline{w_{x}}+\kappa^{2}\right]^{2}}\right]\left(\bar{\psi} i \gamma_{j} \psi\right)\left(\bar{\psi} i \gamma_{j} \psi\right) . }
\end{aligned}
$$

As mentioned before, the index $j=x$ or $y$; there is no index sum here. And we assume the random current disorder variance $g_{x}=g_{y} \equiv g_{j}$ (isotropic).

\section{APPENDIX H: TWO-LOOP VERTEX CORRECTIONS}

At leading order, the generic two-loop diagram has the form pictured below.
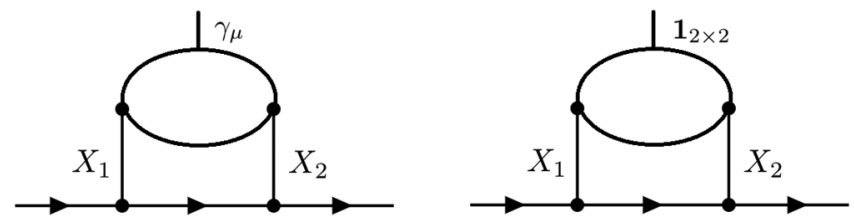

The interaction legs $X_{1}$ and $X_{2}$ can be chosen to be the gauge propagator $G_{\mu \nu}$ or disorder $E_{\mu \nu} \in\left\{W_{\mu \nu}, g_{m}\right\}$. In principal there are four possible choices: $\left(X_{1}, X_{2}\right)=(G, G),(E, G),(G, E)$, or $(E, E)$. In the replica limit $n_{r} \rightarrow 0$, the $(E, E)$ diagram vanishes because the fermion bubble is proportional to $n_{R}$. Also, $(E, G)$ and $(G, E)$ are the same diagrams so we only need to compute one of them. The top vertex can be either $\gamma^{\mu}$ or $\mathbf{1}_{2 \times 2}$. However, we will see below that diagrams using the $\gamma^{\mu}$ vertex are zero.

\section{Mass vertex $\bar{u} 1_{2 \times 2} u$ : One leg gauge, one leg disorder}
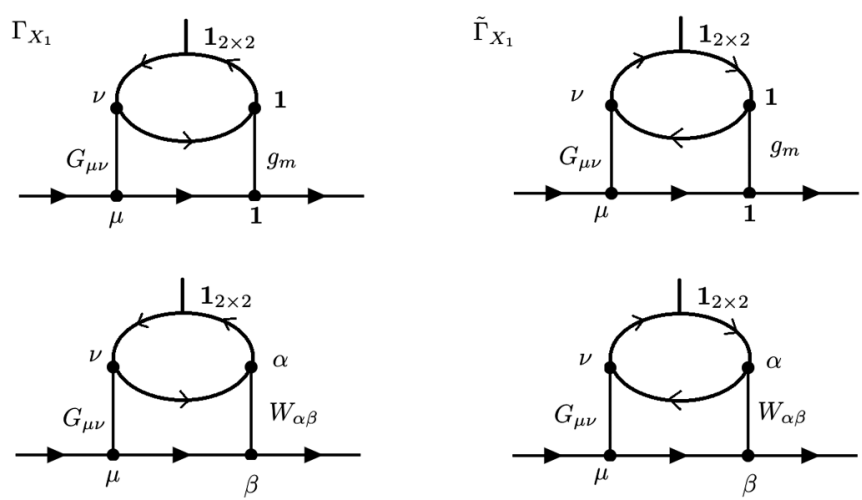

$$
\begin{aligned}
\Gamma_{X_{1}}= & \frac{1}{v^{2}} \int \frac{d^{2} \overline{\boldsymbol{q}} d q_{0}}{(2 \pi)^{3}} \frac{1}{v^{2}} \int \frac{d^{2} \overline{\boldsymbol{k}}}{(2 \pi)^{2}} \bar{u}\left(p_{3}\right)\left(\begin{array}{c}
\mathbf{1} \\
\gamma_{\beta}
\end{array}\right) S_{F}\left(p_{1}-k\right) \frac{-i g}{\sqrt{N_{f}}}\left(\frac{v}{c}\right)^{1-\delta_{\mu 0}} \gamma_{\mu} u\left(p_{1}\right) G_{\mu \nu}(k)(-1) \\
\times & N_{f} \operatorname{Tr}\left[\frac{-i g}{\sqrt{N_{f}}}\left(\frac{v}{c}\right)^{1-\delta_{v 0}} \gamma_{\nu} S_{F}\left(q-p_{1}+p_{3}\right) \mathbf{1} S_{F}(q)\left(\begin{array}{c}
\mathbf{1} \\
\gamma_{\alpha}
\end{array}\right) S_{F}\left(k+q-p_{1}+p_{3}\right)\right]\left(\begin{array}{c}
g_{m} \\
W_{\alpha \beta}\left(k-p_{1}+p_{3}\right)
\end{array}\right) . \\
\tilde{\Gamma}_{X_{1}}= & \frac{1}{v^{2}} \int \frac{d^{2} \overline{\boldsymbol{q}} d q_{0}}{(2 \pi)^{3}} \frac{1}{v^{2}} \int \frac{d^{2} \overline{\boldsymbol{k}}}{(2 \pi)^{2}} \bar{u}\left(p_{3}\right)\left(\begin{array}{c}
\mathbf{1} \\
\gamma_{\beta}
\end{array}\right) S_{F}\left(p_{1}-k\right) \frac{-i g}{\sqrt{N_{f}}}\left(\frac{v}{c}\right)^{1-\delta_{\mu 0}} \gamma_{\mu} u\left(p_{1}\right) G_{\mu \nu}(k)(-1) \\
& \times N_{f} \operatorname{Tr}\left[\frac{-i g}{\sqrt{N_{f}}}\left(\frac{v}{c}\right)^{1-\delta_{v 0}} \gamma_{\nu} S_{F}(q-k)\left(\begin{array}{c}
\mathbf{1} \\
\gamma_{\alpha}
\end{array}\right) S_{F}\left(q-p_{1}+p_{3}\right) \mathbf{1} S_{F}(q)\right]\left(\begin{array}{c}
g_{m} \\
W_{\alpha \beta}\left(k-p_{1}+p_{3}\right)
\end{array}\right) .
\end{aligned}
$$

The direction of the fermionic loop momenta is different in $\Gamma$ and $\tilde{\Gamma}$. We use the upper/lower components to distinguish the diagrams that arise from either $g_{m} / W_{\mu \nu}$. 
To extract the UV divergence, we can set $p_{1}=p_{3}=0$. For $g_{m}$, the divergences in $\Gamma_{X_{1}}$ and $\tilde{\Gamma}_{X_{1}}$ cancel (upon changing variables $q \rightarrow-q$ in $\tilde{\Gamma}_{X_{1}}$ and using basic properties of the trace). For $W_{\alpha \beta}, \Gamma_{X_{1}}$ and $\tilde{\Gamma}_{X_{1}}$ have identical esdivergences:

$$
\begin{aligned}
\Gamma_{X_{1}}= & \frac{-g^{2}}{1}\left(\frac{v}{c}\right)^{2-\delta_{\mu 0}-\delta_{v 0}} \int \frac{d^{3} k}{(2 \pi)^{3}} \bar{u}\left(p_{3}\right)\left[\gamma_{\beta} \frac{k_{0} \gamma_{0}+v k_{c} \gamma_{c}}{k_{0}^{2}+v^{2} k^{2}} \gamma_{\mu}\right] u\left(p_{1}\right) \\
& \times G_{\mu \nu}(k) \times W_{\alpha \beta}(k) \times \int \frac{d^{3} q}{(2 \pi)^{3}} \operatorname{Tr}\left[\gamma_{\nu} \frac{q_{0} \gamma_{0}+v q_{d} \gamma_{d}}{q_{0}^{2}+v^{2} q^{2}} \mathbf{1} \frac{q_{0} \gamma_{0}+v q_{e} \gamma_{e}}{q_{0}^{2}+v^{2} q^{2}} \gamma_{\alpha} \frac{\left(k_{0}+q_{0}\right) \gamma_{0}+v\left(k_{f}+q_{f}\right) \gamma_{f}}{\left(k_{0}+q_{0}\right)^{2}+v^{2}(k+q)^{2}}\right] .
\end{aligned}
$$

Refer to the calculations in (H16) to compute

$$
\int \frac{d^{3} q}{(2 \pi)^{3}} \operatorname{Tr}\left[\gamma_{v} \frac{q_{0} \gamma_{0}+v q_{d} \gamma_{d}}{q_{0}^{2}+v^{2} q^{2}} \frac{q_{0} \gamma_{0}+v q_{e} \gamma_{e}}{q_{0}^{2}+v^{2} q^{2}} \gamma_{\alpha} \frac{\left(k_{0}+q_{0}\right) \gamma_{0}+v\left(k_{f}+q_{f}\right) \gamma_{f}}{\left(k_{0}+q_{0}\right)^{2}+v^{2}(k+q)^{2}}\right]=\frac{i \epsilon_{\nu \alpha \sigma}\left(k_{0}, v \boldsymbol{k}\right)_{\sigma}}{8 v^{2} \sqrt{k_{0}^{2}+v^{2} \boldsymbol{k}^{2}}} .
$$

After setting $g=c=1$,

$$
\begin{aligned}
\Gamma_{X_{1}}= & \frac{-g^{2}}{1}\left(\frac{v}{c}\right)^{2-\delta_{\mu}-\delta_{\nu 0}} \int \frac{d^{3} k}{(2 \pi)^{3}} \bar{u}\left(p_{3}\right)\left[\gamma_{\beta} \frac{k_{0} \gamma_{0}+v k_{c} \gamma_{c}}{k_{0}^{2}+v^{2} k^{2}} \gamma_{\mu}\right] u\left(p_{1}\right) \times G_{\mu \nu}(k) \times W_{\alpha \beta}(k) \times \frac{i \epsilon_{\nu \alpha \sigma}\left(k_{0}, v \boldsymbol{k}\right)_{\sigma}}{8 v^{2} \sqrt{k_{0}^{2}+v^{2} \boldsymbol{k}^{2}}} \\
= & \frac{\left(g_{0} g_{1}-g_{j} g_{1}-g_{j} \overline{w_{x}}\right)}{4 \pi v^{2} \epsilon\left(g_{1}^{2}+g_{1} \overline{w_{x}}+\kappa^{2}\right)}+\phi_{1}\left[\frac{2 g_{1}^{3}\left(g_{1}+\overline{w_{x}}\right)\left(-g_{1} \overline{g_{j}}+g_{1} \overline{g_{0}}+\overline{g_{0}} \overline{w_{x}}\right)}{\epsilon\left(g_{1}^{2}+g_{1} \overline{w_{x}}+\kappa^{2}\right)^{3}}\right. \\
& \left.-\frac{g_{1}^{2}\left[7 \overline{g_{0}}\left(g_{1}+\overline{w_{x}}\right)-\overline{g_{j}}\left(7 g_{1}+4 \overline{w_{x}}\right)\right]}{2 \epsilon\left(g_{1}^{2}+g_{1} \overline{w_{x}}+\kappa^{2}\right)^{2}}+\frac{g_{1}\left(\overline{g_{0}}-\overline{g_{j}}\right)}{\epsilon\left(g_{1}^{2}+g_{1} \overline{w_{x}}+\kappa^{2}\right)}\right] .
\end{aligned}
$$

In total, we need to multiply by a factor of 4 to count the clockwise/counterclockwise fermion loops and the exchange of $W \leftrightarrow G$ in the diagrams:

$$
\Gamma_{X_{1}}+\tilde{\Gamma}_{X_{1}}+\left(\Gamma_{X_{1}}+\tilde{\Gamma}_{X_{1}}\right)_{W \leftrightarrow G}=4 \Gamma_{X_{1}}
$$

\section{Vector vertex $\bar{u} \gamma^{\rho} u$ : One leg gauge, one leg disorder}

Replace the mass-vertex expressions 1 by $\gamma_{\rho}$ :
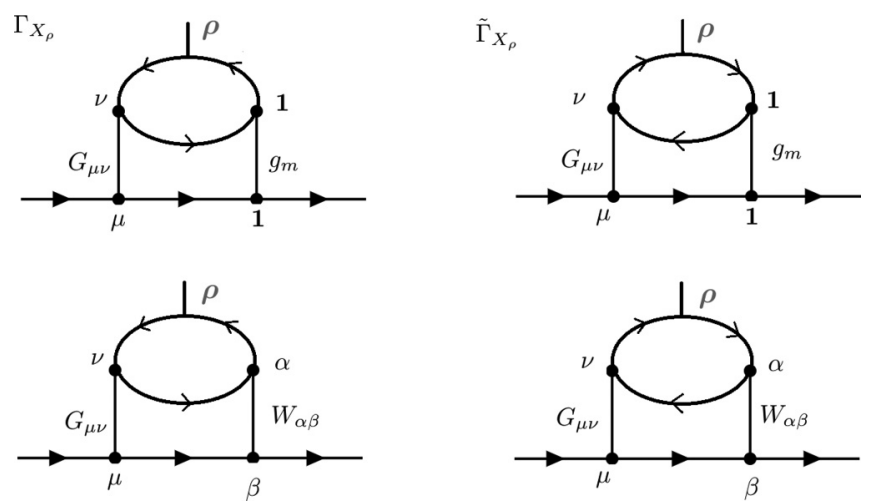

$$
\begin{aligned}
\Gamma_{X_{\rho}}= & \frac{1}{v^{2}} \int \frac{d^{2} \overline{\boldsymbol{q}} d q_{0}}{(2 \pi)^{3}} \frac{1}{v^{2}} \int \frac{d^{2} \overline{\boldsymbol{k}}}{(2 \pi)^{2}} \bar{u}\left(p_{3}\right)\left(\begin{array}{c}
\mathbf{1} \\
\gamma_{\beta}
\end{array}\right) S_{F}\left(p_{1}-k\right) \frac{-i g}{\sqrt{N_{f}}}\left(\frac{v}{c}\right)^{1-\delta_{\mu 0}} \gamma_{\mu} u\left(p_{1}\right) G_{\mu \nu}(k)(-1) \times N_{f} \\
& \times \operatorname{Tr}\left[\frac{-i g}{\sqrt{N_{f}}}\left(\frac{v}{c}\right)^{1-\delta_{v 0}} \gamma_{\nu} S_{F}\left(q-p_{1}+p_{3}\right) \gamma_{\rho} S_{F}(q)\left(\begin{array}{c}
\mathbf{1} \\
\gamma_{\alpha}
\end{array}\right) S_{F}\left(k+q-p_{1}+p_{3}\right)\right]\left(\begin{array}{c}
g_{m} \\
W_{\alpha \beta}\left(k-p_{1}+p_{3}\right)
\end{array}\right), \\
\tilde{\Gamma}_{X_{\rho}}= & \frac{1}{v^{2}} \int \frac{d^{2} \overline{\boldsymbol{q}} d q_{0}}{(2 \pi)^{3}} \frac{1}{v^{2}} \int \frac{d^{2} \overline{\boldsymbol{k}}}{(2 \pi)^{2}} \bar{u}\left(p_{3}\right)\left(\begin{array}{c}
\mathbf{1} \\
\gamma_{\beta}
\end{array}\right) S_{F}\left(p_{1}-k\right) \frac{-i g}{\sqrt{N_{f}}}\left(\frac{v}{c}\right)^{1-\delta_{\mu 0}} \gamma_{\mu} u\left(p_{1}\right) G_{\mu \nu}(k)(-1) \times N_{f} \\
& \times \operatorname{Tr}\left[\frac{-i g}{\sqrt{N_{f}}}\left(\frac{v}{c}\right)^{1-\delta_{v 0}} \gamma_{\nu} S_{F}(q-k)\left(\begin{array}{c}
\mathbf{1} \\
\gamma_{\alpha}
\end{array}\right) S_{F}\left(q-p_{1}+p_{3}\right) \gamma_{\rho} S_{F}(q)\right]\left(\begin{array}{c}
g_{m} \\
W_{\alpha \beta}\left(k-p_{1}+p_{3}\right)
\end{array}\right) .
\end{aligned}
$$


By similar argument, the term with an even number of $\gamma$ 's in the trace would cancel between $\Gamma$ and $\tilde{\Gamma}$, so in this case we only need to compute upper component $\left(g_{m}\right)$. Setting $p_{1}=p_{3}=0$, straightforward calculation gives

$$
\begin{aligned}
\Gamma_{X_{\rho}}= & \left(-N_{f}\right)(-i)^{4}\left(\frac{-i g}{\sqrt{N_{f}}}\right)^{2}\left(\frac{v}{c}\right)^{2-\delta_{\mu 0}-\delta_{v 0}} \frac{1}{v^{2}} \int \frac{d^{3} q}{(2 \pi)^{3}} \frac{1}{v^{2}} \int \frac{d^{2} k}{(2 \pi)^{2}} \\
& \times\left[\bar{u}\left(p_{3}\right) \frac{(-k)^{c}}{k^{2}} \gamma_{c} \gamma_{\mu} u\left(p_{1}\right)\right] \operatorname{Tr}\left[\gamma_{\nu} q \gamma_{\rho} q(q+\not k)\right] \frac{1}{q^{2} q^{2}(k+q)^{2}} G_{\mu \nu}(\boldsymbol{k}, \omega=0) g_{m}=0 .
\end{aligned}
$$

So there is no contribution from $\Gamma_{X_{\rho}}, \tilde{\Gamma}_{X_{\rho}}$

\section{Mass vertex $\bar{u} 1_{2 \times 2} u$ : Both legs are gauge propagators}

$$
\begin{aligned}
\Gamma_{Z_{1}}= & \left(\frac{-i g}{\sqrt{N_{f}}}\right)^{4}\left(\frac{v}{c}\right)^{4-\delta_{\mu 0}-\delta_{\nu 0}-\delta_{\alpha 0}-\delta_{\beta 0}} \times(-1) \times\left(N_{f}\right) \int \frac{d^{3} k d^{3} q}{(2 \pi)^{3}(2 \pi)^{3}} \bar{u}\left(p_{3}\right) \gamma_{\beta} S_{F}\left(p_{1}-k\right) \gamma_{\mu} u\left(p_{1}\right) \\
& \times G_{\mu \nu}(k) \operatorname{Tr}\left[\gamma_{\nu} S_{F}\left(q-p_{1}+p_{3}\right) \mathbf{1} S_{F}(q) \gamma_{\alpha} S_{F}\left(k+q-p_{1}+p_{3}\right)\right] G_{\alpha \beta}\left(k-p_{1}+p_{3}\right), \\
& \times\left(\frac{-i g}{\sqrt{N_{f}}}\right)^{4}\left(\frac{v}{c}\right)^{4-\delta_{\mu 0}-\delta_{\nu 0}-\delta_{\alpha 0}-\delta_{\beta 0}} \times(-1) \times\left(N_{f}\right) \int \frac{d^{3} k d^{3} q}{(2 \pi)^{3}(2 \pi)^{3}} \bar{u}\left(p_{3}\right) \gamma_{\beta} S_{F}\left(p_{1}-k\right) \gamma_{\mu} u\left(p_{1}\right) \\
& \times G_{\mu \nu}(k) \operatorname{Tr}\left[\gamma_{\nu} S_{F}\left(-k+q+p_{1}-p_{3}\right) \gamma_{\alpha} S_{F}(q) \mathbf{1} S_{F}\left(q+p_{1}-p_{3}\right)\right] G_{\alpha \beta}\left(k-p_{1}+p_{3}\right) .
\end{aligned}
$$
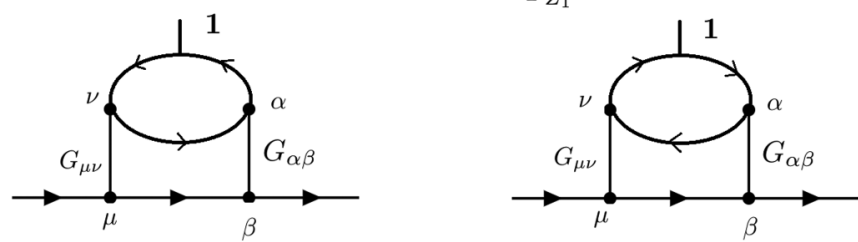

Upon taking the external momenta to zero,

$$
\begin{aligned}
& \Gamma_{Z_{1}}=\frac{g^{4}}{N_{f}}\left(\frac{v}{c}\right)^{4-\delta_{\mu 0}-\delta_{\nu 0}-\delta_{\alpha 0}-\delta_{\beta 0}} \int \frac{d^{3} k}{(2 \pi)^{3}} \bar{u}\left(p_{3}\right)\left[\gamma_{\beta} \frac{k_{0} \gamma_{0}+v k_{c} \gamma_{c}}{k_{0}^{2}+v^{2} k^{2}} \gamma_{\mu}\right] u\left(p_{1}\right) \\
& \times G_{\mu \nu}(k) G_{\alpha \beta}(k) \times \int \frac{d^{3} q}{(2 \pi)^{3}} \operatorname{Tr}\left[\gamma_{v} \frac{q_{0} \gamma_{0}+v q_{d} \gamma_{d}}{q_{0}^{2}+v^{2} q^{2}} \mathbf{1} \frac{q_{0} \gamma_{0}+v q_{e} \gamma_{e}}{q_{0}^{2}+v^{2} q^{2}} \gamma_{\alpha} \frac{\left(k_{0}+q_{0}\right) \gamma_{0}+v\left(k_{f}+q_{f}\right) \gamma_{f}}{\left(k_{0}+q_{0}\right)^{2}+v^{2}(k+q)^{2}}\right] .
\end{aligned}
$$

Perform the $q$ integral first:

$$
\begin{aligned}
F_{\Gamma_{z}}(k) & \equiv \int \frac{d^{3} q}{(2 \pi)^{3}} \operatorname{Tr}\left[\gamma_{\nu} \frac{q_{0} \gamma_{0}+v q_{d} \gamma_{d}}{q_{0}^{2}+v^{2} q^{2}} \frac{q_{0} \gamma_{0}+v q_{e} \gamma_{e}}{q_{0}^{2}+v^{2} q^{2}} \gamma_{\alpha} \frac{\left(k_{0}+q_{0}\right) \gamma_{0}+v\left(k_{f}+q_{f}\right) \gamma_{f}}{\left(k_{0}+q_{0}\right)^{2}+v^{2}(k+q)^{2}}\right] \\
& =\frac{1}{v^{2}} \int \frac{d^{3} Q}{(2 \pi)^{3}} \operatorname{Tr}\left[\gamma_{\nu} \frac{Q_{\lambda} \gamma_{\lambda}}{Q^{2}} \frac{Q_{\rho} \gamma_{\rho}}{Q^{2}} \gamma_{\alpha} \frac{(K+Q)_{\sigma} \gamma_{\sigma}}{(K+Q)^{2}}\right] .
\end{aligned}
$$

Here we define $Q \equiv\left(q_{0}, v \boldsymbol{q}\right), d^{3} Q \equiv d q_{0} d^{2}(v \boldsymbol{q}), K \equiv\left(k_{0}, v \boldsymbol{k}\right)$. Standard Feynman tricks give

$$
F_{\Gamma_{z}}(k)=\frac{i \epsilon_{v \alpha \sigma}\left(k_{0}, v \boldsymbol{k}\right)_{\sigma}}{8 v^{2} \sqrt{k_{0}^{2}+v^{2} \boldsymbol{k}^{2}}} .
$$

So we have $\left(k_{0}=\omega\right)$

$$
\begin{aligned}
\Gamma_{Z_{1}} & =\frac{g^{4}}{N_{f}}\left(\frac{v}{c}\right)^{4-\delta_{\mu 0}-\delta_{\nu 0}-\delta_{\alpha 0}-\delta_{\beta 0}} \int \frac{d^{3} k}{(2 \pi)^{3}} \bar{u}\left(p_{3}\right)\left[\gamma_{\beta} \frac{k_{0} \gamma_{0}+v k_{c} \gamma_{c}}{k_{0}^{2}+v^{2} k^{2}} \gamma_{\mu}\right] u\left(p_{1}\right) G_{\mu \nu}(k) G_{\alpha \beta}(k)\left(\frac{i \epsilon_{\nu \alpha \sigma}\left(k_{0}, v \boldsymbol{k}\right)_{\sigma}}{8 v^{2} \sqrt{k_{0}^{2}+v^{2} \boldsymbol{k}^{2}}}\right) \\
& =\bar{u}\left(p_{3}\right) \mathbf{1}_{2 \times 2} u\left(p_{1}\right) \int_{-\infty}^{\infty} d z \frac{-g^{4} v^{2}\left(\sigma_{e}+|z|\right)\left[\left(v^{2}+z^{2}\right)\left(g_{1}^{2}-\kappa^{2}\right) \sigma_{e}+|z|\left(g_{1} w_{x} \sqrt{v^{2}+z^{2}}+\left(g_{1}^{2}-\kappa^{2}\right)\left(v^{2}+z^{2}\right)\right]\right.}{16 \epsilon N_{f} \pi^{2}\left(v^{2}+z^{2}\right)^{\frac{3}{2}}\left[\sqrt{v^{2}+z^{2}}\left(g_{1}^{2}+\kappa^{2}\right) \sigma_{e}+|z|\left(g_{1}^{2} \sqrt{v^{2}+z^{2}}+\left(g_{1} w_{x}+\sqrt{v^{2}+z^{2}} \kappa^{2}\right)\right)\right]^{2}} .
\end{aligned}
$$

The same manipulations are used in the computations of $\delta_{1}$ and $\delta_{2}$. Note that unlike the case of $\delta_{1}$ and $\delta_{2}$ this term renormalizes $g_{m}$ without any divergent integration, labeled by $\xi$. 
Taking the limit $\sigma_{e}=w_{x}=0$, the expression reduces to

$$
\left.\Gamma_{Z_{1}}\right|_{\sigma_{e}=w_{x}=0}=\bar{u}\left(p_{3}\right) \mathbf{1}_{2 \times 2} u\left(p_{1}\right) \times \frac{-g^{4}\left(g_{1}^{2}-\kappa^{2}\right)}{8 \epsilon N_{f} \pi^{2}\left(g_{1}^{2}+\kappa^{2}\right)^{2}},
$$

which agrees with the result in [25] Unlike the case of $\Gamma_{X_{1}}$, the two legs are identical so the symmetry factor is 2 :

$$
\Gamma_{Z_{1}}+\tilde{\Gamma}_{Z_{1}}=2 \Gamma_{Z_{1}}
$$

\section{Vector vertex $\bar{u} \gamma^{\rho} u$ : Both legs are gauge propagators}

Replace 1 by $\gamma_{\rho}$ to obtain the vector counterparts

$$
\Gamma_{Z_{\rho}}
$$

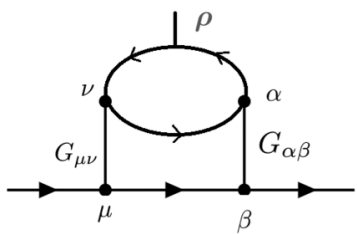

$\Gamma_{Z_{\rho}}=\left(\frac{-i g}{\sqrt{N_{f}}}\right)^{4}\left(\frac{v}{c}\right)^{4-\delta_{\mu 0}-\delta_{\nu 0}-\delta_{\alpha 0}-\delta_{\beta 0}} \times(-1) \times\left(N_{f}\right) \int \frac{d^{3} k d^{3} q}{(2 \pi)^{3}(2 \pi)^{3}} \bar{u}\left(p_{3}\right) \gamma_{\beta} S_{F}\left(p_{1}-k\right) \gamma_{\mu} u\left(p_{1}\right)$

$\times G_{\mu \nu}(k) \operatorname{Tr}\left[\gamma_{\nu} S_{F}\left(q-p_{1}+p_{3}\right) \gamma_{\rho} S_{F}(q) \gamma_{\alpha} S_{F}\left(k+q-p_{1}+p_{3}\right)\right] G_{\alpha \beta}\left(k-p_{1}+p_{3}\right)$,

$\tilde{\Gamma}_{Z_{\rho}}=\left(\frac{-i g}{\sqrt{N_{f}}}\right)^{4}\left(\frac{v}{c}\right)^{4-\delta_{\mu 0}-\delta_{\nu 0}-\delta_{\alpha 0}-\delta_{\beta 0}} \times(-1) \times\left(N_{f}\right) \int \frac{d^{3} k d^{3} q}{(2 \pi)^{3}(2 \pi)^{3}} \bar{u}\left(p_{3}\right) \gamma_{\beta} S_{F}\left(p_{1}-k\right) \gamma_{\mu} u\left(p_{1}\right)$

$\times G_{\mu \nu}(k) \operatorname{Tr}\left[\gamma_{\nu} S_{F}\left(-k+q+p_{1}-p_{3}\right) \gamma_{\alpha} S_{F}(q) \gamma_{\rho} S_{F}\left(q+p_{1}-p_{3}\right)\right] G_{\alpha \beta}\left(k-p_{1}+p_{3}\right)$.

By the same argument as before, there are six $\gamma$ 's in the trace, so $\Gamma_{Z_{\rho}}$ and $\tilde{\Gamma}_{Z_{\rho}}$ cancel one another:

$$
\Gamma_{Z_{\rho}}+\tilde{\Gamma}_{Z_{\rho}}=0
$$

\section{APPENDIX I: THREE-LOOP CORRECTIONS OF DISORDERS $\Delta_{0}$ and $\Delta_{j}$}

1. With the $D_{\alpha \beta}^{\text {dis }}$ propagator

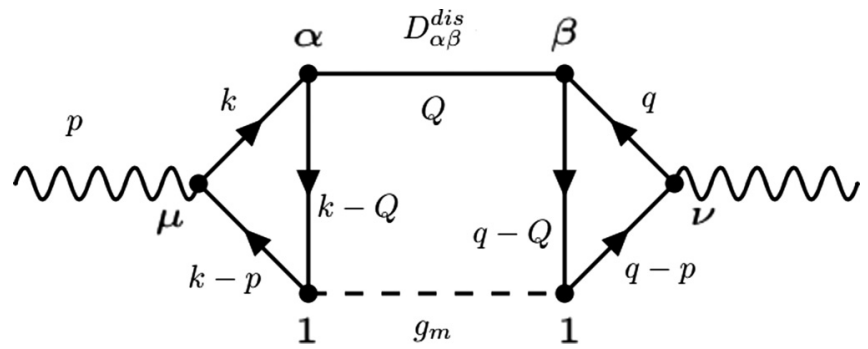

$$
\begin{aligned}
\pi_{1}^{\mu \nu}= & \int \frac{d^{3} k}{(2 \pi)^{3}} \int \frac{d^{3} q}{(2 \pi)^{3}} \int \frac{d^{2} Q d Q_{0}}{(2 \pi)^{2}}\left(\frac{-i g}{\sqrt{N_{f}}}\right)^{4}\left(\frac{v}{c}\right)^{4-\delta_{\mu 0}-\delta_{\nu 0}-\delta_{\alpha 0}-\delta_{\beta 0}}(-1)^{2}\left(N_{f}\right)^{2} \operatorname{Tr}\left[\gamma_{\mu} S_{F}(k-p) \mathbf{1} S_{F}(k-Q) \gamma_{\alpha} S_{F}(k)\right] \\
& \times g_{m} \delta\left(p_{0}-Q_{0}\right) \delta\left(Q_{0}\right) D_{\alpha \beta}^{\mathrm{dis}}\left(\boldsymbol{Q}, Q_{0}=0\right) \times \operatorname{Tr}\left[\gamma_{\nu} S_{F}(q) \gamma_{\beta} S_{F}(q-Q) \mathbf{1} S_{F}(q-p)\right]
\end{aligned}
$$

(flipping the signs for $k$ and $q$ variables)

$$
\begin{aligned}
= & \int \frac{d^{2} Q}{(2 \pi)^{2}}\left[\int \frac{d^{3} k}{(2 \pi)^{3}}\left(\frac{v}{c}\right)^{2-\delta_{\mu 0}-\delta_{\alpha 0}} \operatorname{Tr}\left[\gamma_{\mu} S_{F}(k+p) \mathbf{1} S_{F}(k+Q) \gamma_{\alpha} S_{F}(k)\right]\right] \\
& \times\left[\int \frac{d^{3} q}{(2 \pi)^{3}}\left(\frac{v}{c}\right)^{2-\delta_{\nu 0}-\delta_{\beta 0}} \operatorname{Tr}\left[\gamma_{\nu} S_{F}(q) \gamma_{\beta} S_{F}(q+Q) \mathbf{1} S_{F}(q+p)\right]\right]\left[g_{m}\left(\frac{-i g}{\sqrt{N_{f}}}\right)^{4} N_{f}^{2} D_{\alpha \beta}^{\mathrm{dis}}\left(\boldsymbol{Q}, Q_{0}=0\right) \delta\left(p_{0}=0\right)\right] .
\end{aligned}
$$


Naively evaluating this diagram is problematic because the Feynman parameter integrals are not doable. To extract the divergence, we Taylor expand the expression to second order in $p$. First, we define

$$
T^{\mu \alpha}(Q, p)=\left[\int \frac{d^{3} k}{(2 \pi)^{3}}\left(\frac{v}{c}\right)^{2-\delta_{\mu 0}-\delta_{\alpha 0}} \operatorname{Tr}\left[\gamma_{\mu} S_{F}(k+p) \mathbf{1} S_{F}(k+Q) \gamma_{\alpha} S_{F}(k)\right] .\right.
$$

By reversing the trace order, we have

$$
\begin{aligned}
& {\left[\int \frac{d^{3} q}{(2 \pi)^{3}}\left(\frac{v}{c}\right)^{2-\delta_{\nu 0}-\delta_{\beta 0}} \operatorname{Tr}\left[\gamma_{\nu} S_{F}(q) \gamma_{\beta} S_{F}(q+Q) \mathbf{1} S_{F}(q+p)\right]\right]} \\
& =(-1)^{5}\left[\int \frac{d^{3} q}{(2 \pi)^{3}}\left(\frac{v}{c}\right)^{2-\delta_{\nu 0}-\delta_{\beta 0}} \operatorname{Tr}\left[\gamma_{\nu} S_{F}(q+p) S(q+Q) \gamma_{\beta} S_{F}(q)\right]\right]=-T^{\nu \beta}(Q, p) .
\end{aligned}
$$

Let

$$
\pi_{1}^{\mu \nu}=\int \frac{d^{2} Q}{(2 \pi)^{2}}\left[T^{\mu \alpha}(Q, p)\right]\left[-T^{\nu \beta}(Q, p)\right] \times\left[g_{m}\left(\frac{-i g}{\sqrt{N_{f}}}\right)^{4} N_{f}^{2} D_{\alpha \beta}^{\mathrm{dis}}\left(\boldsymbol{Q}, Q_{0}=0\right) \delta\left(p_{0}=0\right)\right]
$$

and

$$
\begin{aligned}
T_{2}(Q, p) & \equiv T^{\mu \alpha} T^{\nu \beta}, \\
T_{2}(Q, p) & =T_{2}(Q, 0)+\frac{\partial T_{2}}{\partial p_{x}} p_{x}+\frac{\partial T_{2}}{\partial p_{y}} p_{y}+\frac{1}{2}\left[\frac{\partial^{2} T_{2}}{\partial p_{x}^{2}} p_{x}^{2}+\frac{\partial^{2} T_{2}}{\partial p_{y}^{2}} p_{y}^{2}+2 \frac{\partial^{2} T_{2}}{\partial p_{x} p_{y}} p_{x} p_{y}\right]+\mathcal{O}\left(p^{3}\right) \\
& =\left.\left(\frac{\partial T^{\mu \alpha}}{\partial p_{x}} \frac{\partial T^{\nu \beta}}{\partial p_{x}}\right)\right|_{p=0} p_{x}^{2}+\left.\left(\frac{\partial T^{\mu \alpha}}{\partial p_{y}} \frac{\partial T^{\nu \beta}}{\partial p_{y}}\right)\right|_{p=0} p_{y}^{2}+\left.\left(\frac{\partial T^{\mu \alpha}}{\partial p_{x}} \frac{\partial T^{\nu \beta}}{\partial p_{y}}+\frac{\partial T^{\mu \alpha}}{\partial p_{y}} \frac{\partial T^{\nu \beta}}{\partial p_{x}}\right)\right|_{p=0} p_{x} p_{y}+\mathcal{O}\left(p^{3}\right) .
\end{aligned}
$$

Straightforward calculation gives

$$
T^{\mu \alpha}(Q, p=0)=0 .
$$

For first-order derivatives, we can also obtain (after lengthy algebra)

$$
\frac{\partial T^{\mu \alpha}}{\partial p_{j}}=\left(\frac{v}{c}\right)^{2-\delta_{\mu 0}-\delta_{\alpha 0}} \frac{1}{v^{2}} \frac{i(-i)^{3}}{32|\bar{Q}|}\left(\epsilon^{\mu \alpha j}+\frac{1}{\bar{Q}^{2}}\left[\epsilon^{\mu \alpha \tau} \bar{Q}_{\tau} \bar{Q}_{j}+\epsilon^{\alpha j \tau} \bar{Q}_{\tau} \bar{Q}_{\mu}-\epsilon^{j \mu \tau} \bar{Q}_{\tau} \bar{Q}_{\alpha}\right]\right) .
$$

Notice that this result is true in three dimensions with general temporal component $Q_{0}$.

Plugging into Eq. (I2) and taking the four diagrams into consideration (each triangle has either clockwise or counterclockwise flowing momenta), the total result is

$$
\pi_{\mu \nu}^{\mathrm{tot}}=4 \pi_{\mu \nu}
$$

where

$$
\begin{gathered}
\pi_{11}=(-1) \frac{g_{m}\left(g_{1}^{2} \Delta_{0}+v^{2} \Delta_{j} \kappa^{2}\right)}{1024 \pi v^{4} \epsilon\left(g_{1}^{2}+g_{1} \frac{w_{x}}{v}+\kappa^{2}\right)^{2}}\left(\bar{p}_{y}^{2}+\bar{p}_{x}^{2}\right), \\
\pi_{i j}=(+1) \frac{g_{m}\left(g_{1}^{2} v^{2} \Delta_{j}+2 g_{1} v w_{x} \Delta_{j}+w_{x}^{2} \Delta_{j}+\Delta_{0} \kappa^{2}\right)}{512 \pi v^{4} \epsilon\left(g_{1}^{2}+g_{1} \frac{w_{x}}{v}+\kappa^{2}\right)^{2}}\left(\delta_{i j} \bar{p}^{2}-\bar{p}_{i} \bar{p}_{j}\right),
\end{gathered}
$$

and $\bar{p}_{i}=v p_{i} . \pi_{11}$ renormalizes $\Delta_{j}$ and $\pi_{i j}$ renormalizes $\Delta_{0}$. This diagram scales as $1 / N_{f}^{2}$ if $g_{m}, \Delta_{0}$, and $\Delta_{j}$ scale as $1 / N_{f}$.

\section{With the $W_{\alpha \beta}$ propagator}

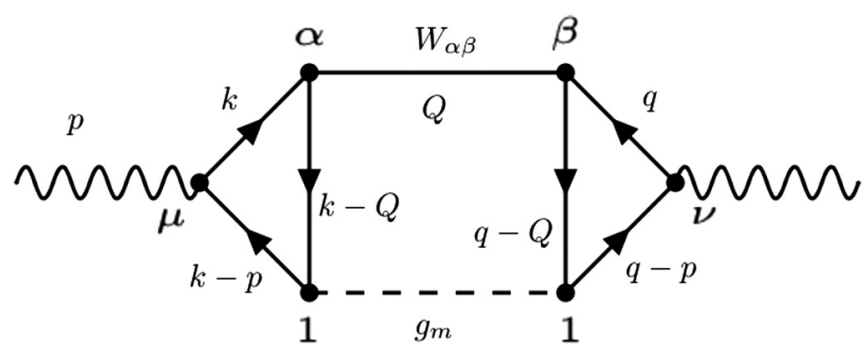


Replacing the internal propagator with $W_{\alpha \beta}$, the remaining calculations are the same:

$$
\tilde{\pi}_{\mu \nu}^{\mathrm{tot}}=4 \tilde{\pi}_{\mu \nu},
$$

where

$$
\begin{gathered}
\tilde{\pi}_{11}=(-1) \frac{g_{m} g_{j} N_{f}}{1024 \pi v^{4} \epsilon}\left(\bar{p}_{y}^{2}+\bar{p}_{x}^{2}\right)+\phi_{1} \frac{g_{1}^{2} g_{m} N_{f}\left(g_{1}^{2} g_{j}+2 g_{1} g_{j} \frac{w_{x}}{v}+\left(2 g_{j}-g_{0}\right) \kappa^{2}\right)}{1024 \pi v^{4} \epsilon\left(g_{1}^{2}+g_{1} \frac{w_{x}}{v}+\kappa^{2}\right)^{2}}\left(\bar{p}_{y}^{2}+\bar{p}_{x}^{2}\right), \\
\tilde{\pi}_{i j}=(+1) \frac{g_{0} g_{m} N_{f}}{512 \pi v^{4} \epsilon}\left(\delta_{i j} \overline{\boldsymbol{p}}^{2}-\bar{p}_{i} \bar{p}_{j}\right)+\phi_{1} \frac{g_{1} g_{m} N_{f}\left[g_{1} g_{j} \kappa^{2}-g_{0}\left(g_{1}+\frac{w_{x}}{v}\right)\left(g_{1}^{2}+g_{1} \frac{w_{x}}{v}+2 \kappa^{2}\right)\right]}{512 \pi v^{4} \epsilon\left(g_{1}^{2}+g_{1} \frac{w_{x}}{v}+\kappa^{2}\right)^{2}}\left(\delta_{i j} \overline{\boldsymbol{p}}^{2}-\bar{p}_{i} \bar{p}_{j}\right),
\end{gathered}
$$

and $\bar{p}_{i}=v p_{i}$. $\tilde{\pi}_{11}$ renormalizes $\Delta_{j}$, and $\tilde{\pi}_{i j}$ renormalizes $\Delta_{0}$. This diagram scales as $1 / N_{f}$ if $g_{m}, g_{0}$, and $g_{j}$ scale as $1 / N_{f}$.

\section{With gauge propagator $G_{\alpha \beta}$}

By dimensional analysis, this term should be UV finite:

$$
\begin{gathered}
\left.\approx \int d^{2} Q \frac{1}{Q} \frac{1}{Q} p^{2} \frac{1}{Q}\right|_{Q_{0}=p_{0}} \\
\text { APPENDIX J: SUMMARY } \\
\bar{\delta}_{1} p_{0} \gamma^{0}+\bar{\delta}_{2} v p_{j} \gamma^{j}=\Sigma_{d}+\Sigma_{g}+\Sigma_{b}, \\
\bar{\delta}_{g_{m}}(\bar{\psi} \mathbf{1} \psi)(\bar{\psi} \mathbf{1} \psi)=\operatorname{box}_{11}+2\left(\Gamma_{1}^{m}+\Gamma_{3}^{m}+\Gamma_{4}^{m}+4 \Gamma_{X_{1}}+2 \Gamma_{Z_{1}}\right)+2 \Gamma_{2}^{m}, \\
\bar{\delta}_{g_{0}}\left(\bar{\psi} \gamma^{0} \psi\right)\left(\bar{\psi} \gamma^{0} \psi\right)=\operatorname{box}_{\gamma_{0} \gamma_{0}}+2\left(\Gamma_{1}^{\mu}+\Gamma_{3}^{\mu}+\Gamma_{4}^{\mu}\right)_{\mu=1}\left(\frac{-i g}{\sqrt{N_{f}}}\right)^{-1}+2 \Gamma_{2}^{\mu=1}\left(\frac{-i g}{\sqrt{N_{f}}}\right)^{-1}, \\
\bar{\delta}_{g_{j}}\left(\bar{\psi} i \gamma^{j} \psi\right)\left(\bar{\psi} i \gamma^{j} \psi\right)=\operatorname{box}_{\gamma_{j} \gamma_{j}}+2\left(\Gamma_{1}^{\mu}+\Gamma_{3}^{\mu}+\Gamma_{4}^{\mu}\right)_{\mu=j}\left(\frac{-i g}{\left.\sqrt{N_{f}} v\right)^{-1}+2 \Gamma_{2}^{\mu=j}\left(\frac{-i g}{\sqrt{N_{f}}} v\right)^{-1},}\right.
\end{gathered}
$$

where $\Sigma_{b}, \Gamma_{2}^{m}, \Gamma_{2}^{\mu=1}$, and $\Gamma_{2}^{\mu=j}$ are the sub-leading-order terms in the above expressions:

$$
\begin{gathered}
\bar{\delta}_{\Delta_{0}}\left(\delta_{i j} \boldsymbol{p}^{2}-p_{i} p_{j}\right)=4 \pi_{i j}+4 \tilde{\pi}_{i j}, \\
\bar{\delta}_{\Delta_{j}}\left(p_{x}^{2}+p_{y}^{2}\right)=4 \pi_{11}+4 \tilde{\pi}_{11} .
\end{gathered}
$$

[1] P. A. Lee and T. V. Ramakrishnan, Disordered electronic systems, Rev. Mod. Phys. 57, 287 (1985).

[2] D. Belitz and T. R. Kirkpatrick, The anderson-mott transition, Rev. Mod. Phys. 66, 261 (1994).

[3] A. Lagendijk, B. Van Tiggelen, and D. S. Wiersma, Fifty years of anderson localization, Phys. Today 62(8), 24 (2009).

[4] S. L. Sondhi, S. M. Girvin, J. P. Carini, and D. Shahar, Continuous quantum phase transitions, Rev. Mod. Phys. 69, 315 (1997).

[5] W. Li, G. A. Csáthy, D. C. Tsui, L. N. Pfeiffer, and K. W. West, Scaling and Universality of Integer Quantum Hall Plateau-toPlateau Transitions, Phys. Rev. Lett. 94, 206807 (2005).

[6] W. Li, C. L. Vicente, J. S. Xia, W. Pan, D. C. Tsui, L. N. Pfeiffer, and K. W. West, Scaling in Plateau-to-Plateau Transition: A Direct Connection of Quantum Hall Systems with the Anderson Localization Model, Phys. Rev. Lett. 102, 216801 (2009).

[7] W. Li, J. S. Xia, C. Vicente, N. S. Sullivan, W. Pan, D. C. Tsui, L. N. Pfeiffer, and K. W. West, Crossover from the nonuniversal scaling regime to the universal scaling regime in quantum hall plateau transitions, Phys. Rev. B 81, 033305 (2010).
[8] B. Huckestein, Scaling theory of the integer quantum Hall effect, Rev. Mod. Phys. 67, 357 (1995).

[9] K. Slevin and T. Ohtsuki, Critical exponent for the quantum Hall transition, Phys. Rev. B 80, 041304(R) (2009).

[10] J. T. Chalker and G. J. Daniell, Scaling, Diffusion, and the Integer Quantized Hall Effect, Phys. Rev. Lett. 61, 593 (1988).

[11] B. Huckestein and L. Schweitzer, Relation between the Correlation Dimensions of Multifractal Wave Functions and Spectral Measures in Integer Quantum Hall Systems, Phys. Rev. Lett. 72, 713 (1994).

[12] M. Salehi, H. Shapourian, I. T. Rosen, M.-G. Han, J. Moon, P. Shibayev, D. Jain, D. Goldhaber-Gordon, and S. Oh, QuantumHall to insulator transition in ultra-low-carrier-density topological insulator films and a hidden phase of the zeroth Landau level, Adv. Mater. 31, 1901091 (2019).

[13] N. Mason, Superconductor-Metal-Insulator Transitions in Two Dimensions, Ph.D. thesis, Stanford University, 2001.

[14] B. Huckestein and M. Backhaus, Integer Quantum Hall Effect of Interacting Electrons: Dynamical Scaling and Critical Conductivity, Phys. Rev. Lett. 82, 5100 (1999). 
[15] T. Senthil, D. Thanh Son, C. Wang, and C. Xu, Duality between $(2+1) d$ Quantum Critical Points, Phys. Rep. 827, 1 (2019).

[16] A. W. W. Ludwig, M. P. A. Fisher, R. Shankar, and G. Grinstein, Integer quantum Hall transition: An alternative approach and exact results, Phys. Rev. B 50, 7526 (1994).

[17] F. D. M. Haldane, Model for a Quantum Hall Effect without Landau Levels: Condensed-Matter Realization of the "Parity Anomaly," Phys. Rev. Lett. 61, 2015 (1988).

[18] D. T. Son, Is the Composite Fermion a Dirac Particle?, Phys. Rev. X 5, 031027 (2015).

[19] N. Seiberg, T. Senthil, C. Wang, and E. Witten, A duality web in $2+1$ dimensions and condensed matter physics, Ann. Phys. (NY) 374, 395 (2016).

[20] C. Wang and T. Senthil, Dual Dirac Liquid on the Surface of the Electron Topological Insulator, Phys. Rev. X 5, 041031 (2015).

[21] M. A. Metlitski and A. Vishwanath, Particle-vortex duality of two-dimensional Dirac fermion from electric-magnetic duality of three-dimensional topological insulators, Phys. Rev. B 93, 245151 (2016).

[22] A. Karch and D. Tong, Particle-Vortex Duality from 3D Bosonization, Phys. Rev. X 6, 031043 (2016).

[23] J. Murugan and H. Nastase, Particle-vortex duality in topological insulators and superconductors, J. High Energy Phys. 05 (2017) 159.

[24] M. P. A. Fisher, P. B. Weichman, G. Grinstein, and D. S. Fisher, Boson localization and the superfluid-insulator transition, Phys. Rev. B 40, 546 (1989).

[25] W. Chen, M. P. A. Fisher, and Y.-S. Wu, Mott transition in an anyon gas, Phys. Rev. B 48, 13749 (1993).

[26] M. Barkeshli and J. McGreevy, A continuous transition between fractional quantum Hall and superfluid states, Phys. Rev. B 89, 235116 (2014)

[27] M. Mulligan, Particle-vortex symmetric liquid, Phys. Rev. B 95, 045118 (2017).

[28] J. Ye and S. Sachdev, Coulomb Interactions at Quantum Hall Critical Points of Systems in a Periodic Potential, Phys. Rev. Lett. 80, 5409 (1998).

[29] J. Ye, Effects of weak disorders on quantum hall critical points, Phys. Rev. B 60, 8290 (1999).

[30] P. Goswami, H. Goldman, and S. Raghu, Metallic phases from disordered (2+1)-dimensional quantum electrodynamics, Phys. Rev. B 95, 235145 (2017).

[31] A. Thomson and S. Sachdev, Quantum electrodynamics in $2+1$ dimensions with quenched disorder: Quantum critical states with interactions and disorder, Phys. Rev. B 95, 235146 (2017).

[32] M. S. Foster and A. W. W. Ludwig, Interaction effects on twodimensional fermions with random hopping, Phys. Rev. B 73, 155104 (2006).

[33] H. Yerzhakov and J. Maciejko, Disordered fermionic quantum critical points, Phys. Rev. B 98, 195142 (2018).

[34] S. Dorogovtsev, Critical exponents of magnets with lengthy defects, Phys. Lett. A 76, 169 (1980).

[35] D. Boyanovsky and J. L. Cardy, Critical behavior of $m$ component magnets with correlated impurities, Phys. Rev. B 26, 154 (1982).

[36] I. D. Lawrie and V. V. Prudnikov, Static and dynamic properties of systems with extended defects: Two-loop approximation, J. Phys. C 17, 1655 (1984).
[37] P. B. Weichman and R. Mukhopadhyay, Particle-hole symmetry and the dirty boson problem, Phys. Rev. B 77, 214516 (2008).

[38] H. Goldman, A. Thomson, L. Nie, and Z. Bi, Interplay of interactions and disorder at the superfluid-insulator transition: A dirty two-dimensional quantum critical point, Phys. Rev. B 101, 144506 (2020).

[39] N. Prokof'ev and B. Svistunov, Superfluid-Insulator Transition in Commensurate Disordered Bosonic Systems: Large-Scale Worm Algorithm Simulations, Phys. Rev. Lett. 92, 015703 (2004).

[40] R. Ng and E. S. Sørensen, Quantum Critical Scaling of Dirty Bosons in Two Dimensions, Phys. Rev. Lett. 114, 255701 (2015).

[41] H. Meier and M. Wallin, Quantum Critical Dynamics Simulation of Dirty Boson Systems, Phys. Rev. Lett. 108, 055701 (2012).

[42] T. Vojta, J. Crewse, M. Puschmann, D. Arovas, and Y. Kiselev, Quantum critical behavior of the superfluid-Mott glass transition, Phys. Rev. B 94, 134501 (2016).

[43] M. P. A. Fisher, G. Grinstein, and S. M. Girvin, Presence of Quantum Diffusion in Two Dimensions: Universal Resistance at the Superconductor-Insulator Transition, Phys. Rev. Lett. 64, 587 (1990).

[44] A. Kapitulnik, N. Mason, S. A. Kivelson, and S. Chakravarty, Effects of dissipation on quantum phase transitions, Phys. Rev. B 63, 125322 (2001).

[45] Y. Wang, I. Tamir, D. Shahar, and N. P. Armitage, Absence of Cyclotron Resonance in the Anomalous Metallic Phase in $\mathrm{InO}_{x}$, Phys. Rev. Lett. 120, 167002 (2018).

[46] N. Mason and A. Kapitulnik, Superconductor-insulator transition in a capacitively coupled dissipative environment, Phys. Rev. B 65, 220505(R) (2002).

[47] A. Vishwanath, J. E. Moore, and T. Senthil, Screening and dissipation at the superconductor-insulator transition induced by a metallic ground plane, Phys. Rev. B 69, 054507 (2004).

[48] D. T. Son, Quantum critical point in graphene approached in the limit of infinitely strong coulomb interaction, Phys. Rev. B 75 235423 (2007)

[49] V. Aji and C. M. Varma, Quantum criticality in dissipative quantum two-dimensional $x y$ and Ashkin-Teller models: Application to the cuprates, Phys. Rev. B 79, 184501 (2009).

[50] J. K. Jain, Composite Fermions (Cambridge University Press, Cambridge, England, 2007).

[51] E. Fradkin, Field Theories of Condensed Matter Physics (Cambridge University Press, Cambridge, England, 2013).

[52] T. H. Hansson, V. Oganesyan, and S. L. Sondhi, Superconductors are topologically ordered, Ann. Phys. (NY) 313, 497 (2004).

[53] A. Kapustin and N. Seiberg, Coupling a QFT to a TQFT and Duality, J. High Energy Phys. 04 (2014) 001.

[54] D. F. Mross, J. Alicea, and O. I. Motrunich, Symmetry and Duality in Bosonization of Two-Dimensional Dirac Fermions, Phys. Rev. X 7, 041016 (2017).

[55] W.-H. Hsiao and D. T. Son, Self-dual $v=1$ bosonic quantum Hall state in mixed-dimensional QED, Phys. Rev. B 100, 235150 (2019).

[56] J.-Y. Chen, J. H. Son, C. Wang, and S. Raghu, Exact BosonFermion Duality on a 3D Euclidean Lattice, Phys. Rev. Lett. 120, 016602 (2018). 
[57] E. Witten, SL(2,Z) action on three-dimensional conformal field theories with abelian symmetry, arXiv:hep-th/0307041.

[58] M. Mulligan and S. Raghu, Composite fermions and the fieldtuned superconductor-insulator transition, Phys. Rev. B 93, 205116 (2016).

[59] M. E. Peskin, Mandelstam 't Hooft Duality in Abelian Lattice Models, Ann. Phys. (NY) 113, 122 (1978).

[60] C. Dasgupta and B. I. Halperin, Phase Transition in a Lattice Model of Superconductivity, Phys. Rev. Lett. 47, 1556 (1981).

[61] S. Kachru, M. Mulligan, G. Torroba, and H. Wang, Mirror symmetry and the half-filled Landau level, Phys. Rev. B 92, 235105 (2015).

[62] B. I. Halperin, P. A. Lee, and N. Read, Theory of the half-filled Landau level, Phys. Rev. B 47, 7312 (1993).

[63] A. Altland and B. Simons, Condensed Matter Field Theory (Cambridge University Press, Cambridge, England, 2010).

[64] P. Coleman, Introduction to Many-Body Physics (Cambridge University Press, Cambridge, England, 2016).

[65] A. B. Harris, Effect of random defects on the critical behaviour of Ising models, J. Phys. C 7, 1671 (1974).

[66] V. Borokhov, A. Kapustin, and X.-k. Wu, Topological disorder operators in three-dimensional conformal field theory, J. High Energy Phys. 11 (2002) 049.

[67] S. M. Chester and S. S. Pufu, Anomalous dimensions of scalar operators in $\mathrm{QED}_{3}$, J. High Energy Phys. 11 (2016) 069.

[68] S. Coleman, Aspects of Symmetry (Cambridge University Press, Cambridge, England, 1985).

[69] W. Siegel, Supersymmetric dimensional regularization via dimensional reduction, Phys. Lett. B 84, 193 (1979).

[70] W. Chen, G. W. Semenoff, and Y.-S. Wu, Two loop analysis of nonAbelian Chern-Simons theory, Phys. Rev. D 46, 5521 (1992).

[71] L. V. Avdeev, G. V. Grigorev, and D. I. Kazakov, Renormalizations in Abelian Chern-Simons field theories with matter, Nucl. Phys. B 382, 561 (1992).
[72] S. Giombi, S. Minwalla, S. Prakash, S. P. Trivedi, S. R. Wadia, and $\mathrm{X}$. Yin, Chern-Simons theory with vector fermion matter, Eur. Phys. J. C 72, 2112 (2012).

[73] O. Aharony, G. Gur-Ari, and R. Yacoby, $d=3$ bosonic vector models coupled to Chern-Simons gauge theories, J. High Energy Phys. 03 (2012) 037.

[74] G. 't Hooft, Dimensional regularization and the renormalization group, Nucl. Phys. B 61, 455 (1973).

[75] S. Weinberg, The Quantum Theory of Fields. Vol. 2: Modern Applications (Cambridge University Press, Cambridge, England, 2013).

[76] W. Rantner and X.-G. Wen, Spin correlations in the algebraic spin liquid: Implications for high- $T_{c}$ superconductors, Phys. Rev. B 66, 144501 (2002).

[77] A. Hui, M. Mulligan, and E.-A. Kim, Non-Abelian fermionization and fractional quantum Hall transitions, Phys. Rev. B 97, 085112 (2018).

[78] P.-S. Hsin and N. Seiberg, Level/rank Duality and ChernSimons-Matter Theories, J. High Energy Phys. 09 (2016) 095.

[79] A. Hui, E.-A. Kim, and M. Mulligan, Non-Abelian bosonization and modular transformation approach to superuniversality, Phys. Rev. B 99, 125135 (2019).

[80] A. Agarwal, Antiparticles as Particles: $\mathrm{QED}_{2+1}$ and Composite Fermions at $v=\frac{1}{2}$, Phys. Rev. Lett. 123, 211601 (2019).

[81] K. Damle and S. Sachdev, Nonzero-temperature transport near quantum critical points, Phys. Rev. B 56, 8714 (1997).

[82] G. Gur-Ari, S. Hartnoll, and R. Mahajan, Transport in ChernSimons-matter theories, J. High Energy Phys. 07 (2016) 090.

[83] N. P. Breznay, M. A. Steiner, S. A. Kivelson, and A. Kapitulnik, Self-duality and a Hall-insulator phase near the superconductorto-insulator transition in indium-oxide films, Proc. Natl. Acad. Sci. USA 113, 280 (2016).

[84] M. P. A. Fisher, Quantum Phase Transitions in Disordered Two-Dimensional Superconductors, Phys. Rev. Lett. 65, 923 (1990). 